\title{
Sur une théorie relativiste des fluides thermodynamiques.
}

\author{
Mémoire de Pham Mau Quan (à Paris).
}

\section{INTRODUCTION}

Ce travail vise à la construction d'une théorie relativiste des fluides thermodynamiques, dans le cadre de la Relativité Générale. Il est consacré d'une part à la détermination des fondements d'une telle théorie, d'autre part à l'étude mathématique de la thérie ainsi obtenue.

Nous nous sommes efforcés de tenir le plus grand compte des bases classiques nécessaires à l'intelligence de la théorie et aux applications possibles. Inversement, notre théorie suggêre une modification de certains résultats classiques concernant la thermodynamique des fluides en mouvement.

Cependant, e'est le point de vae relativiste qui nous intéresse icj, et cela pour plusieurs raisons.

Depuis les découvertes d'Ernstern d'une importance capitale pour toute la Physique moderne, les phénomènes de la Gravitation et de l'Electromagnétisme ont fait l'objet de très beaux travaux. Mais, à part ces deux domaines privilégiés, il n'y a $\theta u$ souvent ailleurs que des. essais timides.

Cependant, comme la représentation de la matière s'effectue nécessaire. ment en Relativité Générale par des schémas du type hydrodynamique, l'hydrodynamique relativiste a fait l'objet d'un exposé cohérent et rigoreux. Elle constitue ainsi le premier pas d'une théorie relativiste complète des milieux continus. Il nous est alors apparu qu'il était nécessaire de développer une theorie thermodynamique des fluides en mouvement. D'antre part, les phénomènes relativistes peuvent recevoir ainsi un principe d'évolution dans l'espace-temps, grace à l'extension relativiste de la notion d'entropie.

Il est clair qu' une théorie relativiste des fluides thermodynamiques doit trouver ses bases dans les théories de l'hydrodynamique et de la chaleur.

Les premiers essais d'Einstein, de Planck, consistaient à étudier la nature des grandeurs thermodynamiques telles que la chaleur, la temperature, l'énergie, l'entropie, à travers leur variance par rapport au groupe de Lonentz. Puis, d'autres recherches ont visé à établir les équations du fluide thermodynamique, soit en Relativite restreinte (C. ECKART), soit en Relativité générale (D. vaN Dantzig), mais d'une manière en général peu satisfaisante. 
VAN DaNTzig se borna à l'étude des fluides qu'il nommait parfaitement parfaits, sans conduction de chaleur dans notre langage. Seul C. ECKarT a introduit la conduction de chaleur d'une façon symétrique dans l'expression du tenseur d'impulsion-énergie. Malheureusement, sa généralisation de l'hypothèse de conduction de Fourier se révèle fort peu naturelle: la tem. pérature y est présente elle-même, à côté de ses dérivées. En fait, aucun de ces auteurs n'a effectivement utilisé l'équation de conduction.

Finalement, les études antérieures se révèlent insuffisantes aussi bien en ce qui concerne les principes d' une thermodynamique relativiste, que les fondements $d$ 'une théorie relativiste des fluides qui tienne compte des phénomènes de chaleur.

Aussi, il nous a paru nécessaire de rependre la question à la base.

La thermodynamique classique justifie l'extension du principe de D'Alembers aux fluides dont le potentiel interne par unité de volume est de la forme $\varphi(\rho, \theta)$ où $\rho$ est la densité et $\theta$ la température.

Cependant, dans l'application du principe de D'Alembert généralisé, on procède anx modifications virtuelles du système en deux stades:

a) une modification virtuelle compatible avec les liaisons à l'instant $t$ et qui laisse invariante la température;

b) une modification de la température fixée au moyen de quelques hypothèses thermodynamiques.

On en déduit $q \mathbf{u}^{\prime}$ en tout point du fluide, existe la relation (')

$$
\varphi(\rho, \theta)-\rho \frac{\partial}{\partial \rho} \varphi(\rho, \theta)+p=0
$$

où $p$ représente la pression du fluide. C'est l'équation caractéristique du fluide. Elle fait intervenir en hydrodynamique un nouveau champ scalaire défini par $\theta$. On doit alors faire intervenir ane nouvelle relation demandée à la théorie de la chaleur, en l'espèce l'équation de conduction :

$$
\operatorname{div}(-x \overrightarrow{\operatorname{grad}} \theta)=c \rho \frac{d \theta}{d t}-\frac{l}{\rho} \frac{d \rho}{d t}
$$

La décomposition précédente permet heureusement en Mécanique classique, d'établir les équations générales du fluide. Mais si, selon les principes de la Relativité, on donne une inertie à l'énergie de quelque origine soit-elle, on ne peut par ce procédé, obtenir les équations rigoureuses du fluide. Pour

(1) P. Duнем, Hydrodynamique - Elasticité - Acoustique (A. Hermann, 1891). 
avoir les équations rigoureuses des fluides thermodynamiques, il nous faut encore tenir compte de l'effet de la gravitation.

Ce sont ces raisons qui nous ont amenés à édifier une théorie des fluides thermodynamiques dans le cadre de la Relativité Générale. Les théories classiques de I'Hydrodynamique et de la Chaleur nous serviront comme point de départ de façon à suivre sans trop de difficulté l'interprétation physique.

Il nous apparaît comme suffisant de prendre pour éléments primitifs de notre théorie, les éléments suivants:

10. - un espace-temps de la Relativité Générale qui fait intervenir la gravitation dans les phénomènes étudiés;

20. - les équations d'EInsters

$$
S_{\alpha \beta}=\chi T_{\alpha \beta}
$$

qui etablissent le lien logique entre le champ gravitationnel inclus dans les propriétés géométriques de l'espace-temps, et le champ de la matière qui constitue le siège des phénomènes thermodynamiques;

$3^{\circ}$. - le phénomène élémentaire de conduction thermique traduit par l'hypothèse de FourIER

$$
\vec{q}=-x \operatorname{grad} \theta
$$

et le principe de Carnot qui conduit à la notion d'entropie.

La variété espace-temps de la Relativité générale possède une structure locale d'espace-temps de Minkowski. Cette propriété fournit Ia méthode pour établir les équations de notre théorie. Elle permet inversement leur interprétation physique et mécanique locale.

En effet, en chaque point de l'espace-temps, existe un repère galiléen local par rapport anquel la matière est an repos au point considéré. Ce repère est appelé repère propre. Il suffit d'écrire dans ce repère, les équations relatives à la matière au repos. Par un changement de repère, on en déduit l'expression générale invariante des équations relativement à un système de coordonnées: curvilignes locales quelconques.

Cette méthode très simple présente quelque analogie avec le principe de D'Alembers en Dynamique elassiqne. Elle nous a permis, on partant des données de l'expérience, de construire toute la base axiomatique de notre théorie.

Nous avons ensuite êtudié, à l'aide d'une analyse du problème de CAUChy, la structure des équations fondamentales du champ. Les résultats de cette étude montrent que la théorie construite est cohérente et que les équations choisies sont satisfaisantes. 


\section{NOTATIONS ET SYMBOLES}

$\alpha, \beta, \ldots, \lambda:$ tout indice grec prend les valeurs $0,1,2,3$.

$i, j, \ldots, l$ : tout indice latin prend les valeurs $1,2,3$.

$\boldsymbol{x}^{\alpha}$ : système de coordonnées curvilignes quelconque.

$f\left(x^{x}\right)$ : fonction scalaire de $x^{x}$.

$\vec{v}$ : vecteur.

$T^{\alpha \beta}, T_{\beta}^{\alpha}, T_{\alpha \beta}$ tenseurs.

Indices superieurs: indices contravariants.

Indiẹs inférieurs: indices covariants.

Convention d'Einstein: Toutes les fois que dans un monôme figure deux fois le mème indice, une fois en indice supérieur et une fois en indice inférieur, on doit, sauf avis contraire, sommer tous les monômes obtenus en donnant à cet indice toutes les valeurs possibles.

Exemples :

$$
\begin{gathered}
\overrightarrow{d \vec{x}}=\omega^{\alpha} \overrightarrow{e_{\alpha}}=\sum_{\alpha=0}^{3} \omega^{\alpha} \overrightarrow{e_{\alpha}} \\
R=R_{\alpha}^{\alpha}=\sum_{\alpha=0}^{3} R_{\alpha}^{\alpha} .
\end{gathered}
$$

Forme quadratique foudamentale

$$
d s^{2}=g_{\alpha \beta}\left(x^{\lambda}\right) d x^{\alpha} d x^{\beta}
$$

$g_{\alpha \beta}$ est le tenseur métrique fondamental ou tenseur de gravitation.

$g^{\alpha \beta}$ est son tenseur conjugué.

$[\alpha \beta, \gamma]$ : symbole de Christoffel de 1ère èspèce

$$
[\alpha \beta, \gamma]=\frac{1}{2}\left(\partial_{\alpha} g_{\beta \gamma}+\partial_{\beta} g_{\alpha \gamma}-\partial_{\gamma} g_{\alpha \beta}\right)
$$

$\left\{\begin{array}{c}\gamma \\ \alpha \beta\end{array}\right\}=\Gamma_{\alpha \beta}^{\gamma}=g^{\delta}[\alpha \beta, \delta]$ : symbole de ChristofFel de seconde espèce.

$\partial_{\alpha}=\frac{\partial}{\partial x^{\alpha}}, \partial_{\alpha \beta}=\frac{\partial^{2}}{\partial x^{\alpha} \partial x^{\beta}}$, dérivées partielles.

$\nabla_{\alpha}, \nabla_{\alpha \beta}, \ldots$, dérivées covariantes.

$\triangle_{1} f \equiv g^{\alpha \beta \partial_{\alpha}} f \partial_{\beta} f$, premier paramètre différentiel de BeLTRAMI.

$\triangle_{2} f \equiv g^{\alpha \beta}\left(\partial_{\alpha \beta} f-\Gamma_{a}{ }^{\rho} \partial_{\rho} f\right)$, second paramètre différentiel de BeLTRAMI ou laplacien de la fonction $f$.

$R_{\alpha \beta}$ est le tenseur de RICcI de la variété riemannienne $V_{4}$, déduit du tenseur de courbure de RIEMANN-CHRISTOFFEL

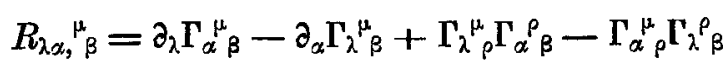

par contraction des indices $\lambda$ et $\mu$ :

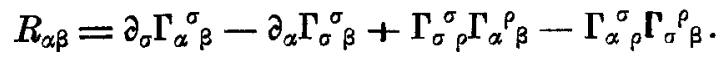

Par contraction de $R_{\alpha \beta}$, on obtient l'invariant

$$
R=g^{\alpha \beta} R_{\alpha \beta} \text {. }
$$




\section{CHAPITRE I.}

\section{CONSIDERATIONS GEOMETRIQUES GENERALES}

\section{La variété espace-temps.}

Dans la théorie de la relativité générale, l'élément géométrique est l'espace-temps. C'est une varieté à quatre dimensions $V_{4}$, douée d'une structure de variété différentiable $q u$ 'il est utile de préciser.

$R^{n}$ désigne l'espace numérique des variables réelles $x^{\alpha}$.

Une fonction $\varphi$ à valeurs numériques définie dans $R^{n}$ est dite de classe $C^{u}$, si elle admet des dérivées partielles continues jusqu'à l'ordre $u$.

Une fonction $\psi$ définie dans un domaine $D$ de $R^{\prime \prime}$ sera dite de classe $C^{v}$ par morceaux, si elle admet la classe $C^{v}$ dans $D$, excepté sur des surfaces de discontinuité $S$ en nombre fini. Dans chacun des voisinages d' une surface $S$ :

1. - $\psi$ est une fonction de classe $C^{v}$.

2. - ses dérivées partielles jusqu'à l'ordre $v$ tendent uniformément vers des limites déterminées différentes de part et d'antre en chaque point de $S$.

La variêté espace-temps $V_{4}$ satisfait aux hypothèses suivantes:

a. - $V_{+}$est un espace topologique connexe dont chaque point possède un voisinage homéomorphe à l'intérieur d'une sphère de l'espace euclidien à quatre dimensions.

b. - Les systèmes "de coordonnées $\left(x^{0}, x^{1}, x^{2}, x^{3}\right)$ dans $V_{4}$ forment une famille $A$, satisfaisant aux deux conditions suivantes:

$A 1$. - Leurs domaines recouvrent entièrement $V_{4}$.

$A$. - Un point $x \in V_{4}$ variant dans la partie commune aux domaines de deux systèmes de la famille $A$, les coordonnées de $x$ relativement à l' un des systèmes, sont des fonctions de classe $C^{2}$ à jacobien non nul, de ses coordonnées relativement à l'autre système.

Les problèmes fondamentaux de la théorie exigent en outre l'hypothèse suivante qui complète $A^{*}$ :

Dans l'intersection des domaines de deux systèmes de coordonnées, les dérivées secondes du changement de coordonnées sont des fonctions de classe $C^{2}$ par morceąux.

Il résulte de ces hypothèses qu' une fonction $f(x)$ définie sur $V_{4}$, peut etre de classe $C^{u}(u=2)$, et que les composantes $d$ ' un tensenr dans $V_{4}$ peuvent être des fonctions de classe $C^{r}(r \leq 1)$ et de classe $C^{r+2}$ par morceaux.

Sur la variété $V_{4}$, nous supposons définie une métrique riemannienne $d s^{2}$, partout $d u$ type hyperbolique normal, par la donnée d'un champ de tenseurs covariants symétriques a deux indices $g_{x \beta}$, appelé tenseur fondamental de gravitation. Soit

$$
d s^{2}=g_{x \beta}\left(x^{\lambda}\right) d x^{\alpha} d x^{\beta} \quad(\alpha, \beta, \lambda=0,1,2,3) .
$$


Les coefficients $g_{\alpha \beta}$ sont appelés les potentiels de gravitation. Ce sont des fonctions de classe $C^{4}$ et leurs dérivées $\partial_{y} g_{a \beta}$ sont des fonctions de classe $C^{2}$ par morceaux.

L'hypothèse faite sur le type hyperbolique normal de la métrique de $V_{4}$, veut dire qu'en chaque point $x \in V_{4}$, le $d s^{2}$ peut se mettre localement sous la forme

$$
d s^{2}=\left(\omega^{0}\right)^{2}-\left(\omega^{1}\right)^{2}-\left(\omega^{2}\right)^{2}-\left(\omega^{3}\right)^{2}
$$

où les $\omega^{z}$ forment un système de formes de PFAFF locales linéairement indépendantes.

\section{Orientation dans l'espace et le temps.}

L'équation

$$
d s^{2}=0
$$

définit en chaque point $x$ de $V_{4}$, un cône réel $\Gamma_{x}$ de directions tangentes à $V_{4}$. Ce cône sera dit cône élémentaire en $x$. L'intérieur du cône $\Gamma_{x}$ est défini tel que $d s^{2}>0$; l'extérieur du cône est tel que $d s^{2}<0$.

1. - Une direction $\overrightarrow{d x}$ est dite orientée dans le temps ou dans l'espace selon qu' elle est intérieure ou extérieure au cône élémentaire. Si la direction $\overrightarrow{d x}$ est orientée dans le temps:

$$
g_{\alpha \beta} d x^{\alpha} d x^{\beta}>0
$$

si elle est orientée dans l'espace :

et réciproquement.

$$
g_{\alpha \beta} d x^{\alpha} d x^{\beta}<0
$$

Une ligne $C$ est dite orientée dans le temps, si les tangentes en tous ses points sont orientées dans le temps. On a alors, le long de $C$ :

$$
d s^{2}=g_{\alpha \beta} d x^{\alpha} d x^{k}>0 \text {. }
$$

La ligne $C$ est dite orientée dans l'espace, si $d s^{2}<0$.

2. - Un 2 ou 3-plan élémentaire tangent en $x$ à $V_{4}$, est dit orienté dans l'espace, si tontes ses directions sont orientées dans l'espace. Il est orienté dans le temps, s' il admet des directions orientées dans le temps.

Si nous considérons une direction $v_{a}$, le 3-plan élémentaire $\Pi_{x}$ qui lui est perpendiculaire, est défini par l'équation

$$
v_{\alpha} d x^{\alpha}=0 \text {. }
$$

La direction $v_{a}$ est conjuguée du 3-plan $\Pi_{x}$, par rapport au cône élémentaire $\Gamma_{x}$. Si $\square_{x}$ est orienté dans l'espace, la direction $v_{x}$ est orientée dans le temps et réciproquement. Il en résulte que $\Pi_{x}$ est orienté dans l'espace, si

$$
g^{\alpha \beta} v_{\alpha} v_{\beta}>0
$$


et que $\Pi_{x}$ est orienté dans le temps, si :

$$
g^{\alpha \beta} v_{\alpha} v_{\beta}<0 \text {. }
$$

Une hypersurface $S$ de $V_{4}$ est un espace à trois dimensions plongé dans $V_{4}$. Elle est dite orientée dans l'espace, si tous ses éléments plans tangents aux différents points, sont orientés dans l'espace. Si l'hypersurface $S$ est définie localement par l'équation :

$$
f\left(x^{\lambda}\right)=0
$$

l'élément-plan tangent en un point $x$ de $S$, est perpendiculaire à la direction $\partial_{\alpha} f$. Par conséquent, si l'hypersurface $S$ est orientee dans l'espace, on a en tout point de $S$ :

$$
\triangle_{1} f \equiv g^{\alpha \beta} \partial_{\alpha} f \partial_{\beta} f>0
$$

L'hypersurface $S$ sera orientée dans le temps si on a sur $S: \triangle_{t} f<0$ partout.

3. Temps et espace associés. Composante de temps et composante d' espace d'un vecteur.

Soit $\vec{\eta}$ un vecteur unitaire orienté dans le temps, et défini en un point $x$ de $V_{4}$.

$$
(\vec{\eta})^{2}=+1
$$

Le tri-plan élémentaire $\mathrm{II}_{x}$, d'équation

$$
\eta_{x} d x^{x}=0
$$

est orthogonal au vecteur $\vec{\eta}$ et orienté dans l'espace.

Nous dirons que $\vec{\eta}$ et $\Pi_{x}$ définissent en $x$, un temps et un espace associés.

Un vecteur quelconque $\vec{X}$ defini en $x$, a par rapport à $\vec{\eta}$ et $\Pi_{x}$, une composante colinéaire $\mathfrak{a} \vec{\eta}$ :

$$
(\vec{X} \cdot \vec{\eta}) \vec{\eta}
$$

dite composante de temps, et une composante $\vec{X}^{*}$ dans $\Pi_{x}$ perpendiculaire à $\vec{\eta}$

$$
\vec{X}^{*} \cdot \vec{\eta}=0
$$

dite composante d'espace de $\vec{X}$.

Le vectéur $\vec{X}$ se trouve ainsi décomposé comme la somme de ces deux composantes :

$$
\vec{X}=(\vec{X} \cdot \vec{\eta}) \vec{\eta}+\vec{X}^{*} .
$$

A toute décomposition en carrés de la métrique de $V_{4}$ :

$$
d s^{2}=\left(\omega^{0}\right)^{2}-\left(\omega^{1}\right)^{2}-\left(\omega^{2}\right)^{2}-\left(\omega^{3}\right)^{2}
$$

correspondent une base $\left(\omega^{0}, \omega^{t}\right)$ pour les formes linéaires locales definies en $x$, et un repère $\left(x ; \overrightarrow{e_{11}}, \overrightarrow{e_{i}}\right)$ pour l'espace tangent $T_{x}$ en $x \cdot \grave{a} V_{4}$. L'indice $i$ prend les valeurs $1,2,3$. 
Nous avons

et

$$
d \vec{x}=\omega^{x} \overrightarrow{e_{\alpha}}
$$

$$
d s^{*}=\left(\overrightarrow{d \vec{x})^{2}}=\left(\omega^{x} \overrightarrow{e_{\alpha}}\right) \cdot\left(\omega^{2} \overrightarrow{e_{\beta}}\right)=\left(\omega^{\alpha} \omega^{\beta}\right) \overrightarrow{e_{\alpha}} \cdot \overrightarrow{e_{\beta}} .\right.
$$

La comparaison de cette égalité à la décomposition (1.2) donne:

$$
\begin{gathered}
\left(\overrightarrow{e_{\mathrm{u}}}\right)^{2}=+1 \\
\left(\overrightarrow{e_{i}}\right)^{2}=-1 \\
\overrightarrow{e^{x}} \cdot \overrightarrow{e^{\beta}}=0 \text { si } \alpha \neq \beta .
\end{gathered}
$$

Un tel repère $\left(\vec{e}_{0}, \vec{e}_{i}\right)$ est dit repère orthonormé en $x$.

Le vecteur $\overrightarrow{e_{0}}$ est orienté dans le temps. Les trois vecteurs $\overrightarrow{e_{i}}$ sont orientés dans l'espace. Ils définissent un 3-plan $\left(\vec{e}_{i}\right)$ orthogonal à $\overrightarrow{e_{0}}$ et orienté dans l'espace. Le vecteur $\overrightarrow{e_{0}}$ et le tri-plan $\overrightarrow{\left(e_{t}\right)}$ définissent donc un temps et un espace associés au point $x$ de $\nabla_{4}$.

La décomposition (1.2) montre que la métrique $d s^{2}$ introduite, définit dans $V_{4}$ une structure d'espace de Minkowskr. Rapporté à an repère orthonormé, l'espace de Minkowski tangent $T_{x}$ doit être identifié à un espacetemps de la relativité restreinte, rapporté à un repère galiléen. Sa considération est fort utile.

Ainsi, un tenseur peut etre défini en un point $x$ de $V_{q}$ par ses composantes relativement à un repère galiléen local. Ses composantes générales quelconques se déduisent des premières par des formules de transformation connues.

Réciproquement, l'interprétation d'an tenseur défini en un point $x$ de $V_{4}$, se dédait de l'espace de MrNKowski tangent. On aura ainsi les interprétations physiques en termes de temps et d'espace.

\section{Utilisation d' un repère galiléen local.}

Soit le repère galiléen local $\left(x, \overrightarrow{\left.V^{(} \lambda^{\prime}\right)}\right)$. Introduisons les symboles suivants:

$$
\delta_{\lambda^{\prime} \mu^{\prime}}=\overrightarrow{V^{\left(\lambda^{\prime}\right)}} \cdot \vec{V}\left(\mu^{\prime \prime}\right)=\left\{\begin{array}{rll}
+1 & \text { si } & \lambda^{\prime}=\mu^{\prime}=0 \\
-1 & \text { si } & \lambda^{\prime}=\mu^{\prime}=i \\
0 & \text { si } & \lambda^{\prime} \neq \mu^{\prime} .
\end{array}\right.
$$

Supposons qu' on rapporte l'espace-temps $V_{4}$ à un repère quelconque $\left(x, \overrightarrow{e_{\lambda}}\right)$ qui pourra etre en particulier le repère naturel en $x$ associé à un système de coordonnées curvilignes locales. La matrice du changement de repère est notée $\left(A_{\alpha}^{\lambda^{\prime}}\right)$. Nous avons les formules de transformation :

$$
g_{\alpha \beta}=A_{\alpha}^{\lambda^{\prime}} A_{\beta}^{\mu^{\prime}} \delta_{\lambda^{\prime} \mu^{\prime}} \text {. }
$$

Les $A_{\alpha}^{\lambda^{\prime}}$ ne sont autre chose que les composantes $V_{\alpha}^{\left(\lambda^{\prime}\right)}$ du vecteur $\overrightarrow{V^{\left(\lambda^{\prime}\right)}}$ dans le repère naturel. 
Nous en déduisons l'expression des composantes du tenseur fondamental $g_{\alpha \beta}$. en fonction des composantes des vecteurs $\vec{T}^{\left(\lambda^{\prime}\right)}$ du repère galiléen local:

$$
g_{\alpha \beta}=V_{\alpha}^{(0)} V_{\beta}^{(0)}-\sum_{i=1}^{3} V_{\alpha}^{(i)} V_{\beta}^{(i)}
$$

Si $\vec{X}$ est un vecteur quelconque defini en $x$, sa composante d'espace $\vec{X}^{*}$ relativement au vecteur unitaire orienté dans le temps $\vec{V}^{(0)}$, a pour valeur:

$$
\vec{X}^{*}=\vec{X}-\left(\vec{X} \cdot \overrightarrow{V^{(0)}}\right) \vec{V}^{(0)} \text {. }
$$

On en deduit les composantes de $\vec{X}^{*}$ dans le repère naturel

$$
X^{* \lambda}=X^{\lambda}-\left(X^{\alpha} V_{\alpha}^{(0)}\right) V^{(0) \lambda} \text {. }
$$

Elles s' écrivent encore :

$$
X^{* \lambda}=X^{\alpha}\left(g_{\alpha}^{\lambda}-V_{\alpha}^{(0)} V^{(0) \lambda}\right) .
$$

On vérifie par les égalités (4.1) que

$$
X^{* \lambda}=-\sum_{i=1}^{3}\left(V_{x}^{(i)} X^{a}\right) V^{(i) \lambda} .
$$

Nous appellerons grandenr d' espace du vecteur $\vec{X}$, le carré de sa composante d'espace $\vec{X}^{*}$. Elle a pour valeur :

$$
\left(\vec{X}^{*}\right)^{2}=g_{\lambda \mu} X^{\lambda} X^{\mu}-\left(X^{\lambda} V_{\lambda}^{(0)}\right)^{2}
$$

C'est une quantité négative ou nulle.

Nous appellerons grandeur de temps du vecteur $\vec{X}$, le carré de sa composante de temps. Elle a pour valeur

$$
\left(X^{\lambda} V_{\lambda}^{(0)}\right)^{2}
$$

5. Le système des équations d' Kinstein.

En relativité générale, les propriétes géométriques de l'espace-temps rendent compte des phénomènes purement gravitationnels. Le premier pas de la théorie générale du fluide (supposé représenter la matière et l'énergie), consiste à choisir un système tensoriel d'equations aux dérivées partielles qui limitent la généralité des potentiels $g_{\alpha \beta}$ du tenseur fondamental de gravitation, en le reliant aux distributions énergétiques dans l'espace-temps.

Dans le cas gravitationnel pur, ces équations devront généraliser l'équation de Laplace-Poisson qui détermine localement le potentiel newtonien.

Des considérations dues à EINSTEIN ont conduit à adopter le système d'équations

$$
S_{\alpha \beta}=\chi T_{\alpha \beta}
$$

où $S_{\alpha \beta}$ et $T_{\alpha \beta}$ sont deux tenseurs symétriques et $\chi$ désigne un facteur constant. 
Le tenseur $T_{\alpha \beta}$ de signification purement physique, doit décrire an mieux, en chaque point de l'espace-temps $V_{4}$, l'état de la distribution énergétique

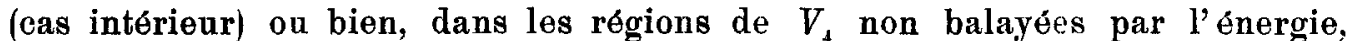
doit être identiquement nul (cas extérieur). Ce tenseur sera appelé tenseur d'impulsion-énergie ou plus brièvement tenseur d'énergie.

Le tenseur $S_{\alpha \beta}$ de signification essentiellement géométrique, est astreint aux conditions suivantes:

1. - il ne dépend que des potentiels $g_{\alpha \beta}$ et de lenrs dérivées des denx premier ordres, et doit être linéaire par rapport aux dérivées $d u$ second ordre.

2. - il est conservatif, e'est-à-dire satisfait aux quatre conditions suivantes appelées conditions de conservation

$$
\nabla_{\alpha} S^{\alpha \beta}=0
$$

$\nabla_{x}$ désigne l'opérateur de dérivation covariante dans $V_{4}$.

Elte Cartan a démontré que les seuls tenseurs $S_{x \beta}$ satisfaisant à ces conditions, sont données par la formule

$$
S_{\alpha \beta}=h\left[R_{\alpha \beta}-\frac{1}{2}(R+k) g_{\alpha \beta}\right]
$$

où $R_{\alpha \beta}$ est le tenseur de Ricci de la variété riemannieñne $V_{4}$.

$$
R_{\alpha \beta}=\partial_{\rho} \Gamma_{\alpha \beta}{ }^{\rho}-\partial_{\alpha} \Gamma_{\rho \beta}{ }^{\rho}+\Gamma_{\sigma \rho}^{\sigma} \Gamma_{\alpha \beta}{ }^{\rho}-\Gamma_{\alpha}{ }^{\sigma} \Gamma_{\sigma \beta}{ }^{\rho}
$$

et $R$, l' invariant $g^{\alpha \beta} R_{\alpha \beta}$. $h$ et $k$ sont deux constantes arbitraires.

En supprimant le facteur surabondant $h$, les équations aux dérivées partielles (5.1) correspondantes, s'écrivent:

$$
S_{\alpha \beta} \equiv R_{\alpha \beta}-\frac{1}{2}(R+k) g_{\alpha \beta}=\chi T_{\alpha \beta} .
$$

La constante $k$, appelée constante cosmologique, n'intervient guère que dans les études de cosmologie. Comme les problèmes d'ordre mathématique restent les mêmes, nous raisonnerons sur les équations:

$$
S_{\alpha \beta} \equiv R_{\alpha \beta}-\frac{1}{2} R g_{\alpha \beta}=\chi T_{\alpha \beta}
$$

qui sont celles du cas intérieur, et les équations

$$
S_{\alpha \beta} \equiv R_{\alpha \beta}-\frac{1}{2} R g_{\alpha \beta}=0
$$

qui sont celles du cas extérieur.

Les équations (5.4), (5.5) seront respectivement appelées les équations d' EInstein du cas intérieur et du eas extérieur. 
CHA PITRE II.

\section{LE FLUIDE THERMODYNAMIQUE RELATIVISTE AXIOMATIQUE ET EQUATIONS GENERALES}

\section{I. - Défnnitions générales.}

\section{Définition ét propriétés généra.les.}

Un fluide relativiste peut être considéré comme un milieu matériel continu, déformable, doué des propriétés suivantes:

1. - il possède une densité propre $\rho$.

2. - il est possible de définir un vecteur vitesse unitaire $\vec{u}$ pour chaque point du milieu.

Nous dirons plus brièvement fluide pour fluide relativiste.

Du point de vue physique, le fluide est généralement doué de propriétés diverses. Chacune de ces propriétés peut se traduire par les valeurs de certaines quantités scalaires ou tensorielles qu'on appelle caractéristiques du fluide.

Ainsi les forces de liaisons internes entre divers éléments dn fluide, sont susceptibles d'être représentées par un tenseur symétrique à deux indices $\pi_{x \beta}$. Ce tenseur est dit tenseur des pressions propres. Il joue un rôle fondamental dans les considérations dynamiques ou thermodynamiques sur le fluide.

Le fluide est le siège de phénomènes physiques correspondant à la chaleur, au rayonnement, à l'électromagnétisme... Il convient de définir dans chaque cas des caractéristiques appropriées. Ainsi les phénomènes électromagnétiques sont susceptibles d'être représentés par un tenseur antisymétrique à deux indices $F_{\alpha \beta}$, dit tenseur champ electromagnétique. Ainsi les propriétés thermodynamiques du fluide s'expriment à l'aide d'un champ scalaire $\theta$, dit ehamp de température.

Le fluide envisagé est dit thermodynamique, lorsque les phénomènes calorifiques ne sont pas négligés. Un tel flnide fait intervenir non seulement les données habituelles du fluide relativiste: vecteur vitesse unitaire $\vec{u}$, densité propre $\rho$, tenseur des pressions propres $\pi_{\alpha \beta}$, mais encore un champ sca. laire $\theta$ dit champ des températures propres.

Dans notre étude, nous nous limiterons au cas des fluides isotropes, dépourvus de tout phénomène chimique. Au cours des raisonnements, il est sous-entendu que les diverses grandeurs physiques sont exprimées en unités convenables, dans un système d'unités où la vitesse limite de propagation des phénomènes physiques est prise comme unité. Les quantités de chaleur s'exprimeront en unités d'énergie. 
La fonction scalaire $\theta$ qui représente la température est supposée de classe $\left(C^{2}, C^{4}\right.$ par morceaux $)$.

\section{Espace-temps riemannien associé à un fluide.}

Dans la théorie générale des fluides thermodynamiques qui sera dévelop. pée, nous représenterons le fluide considéré par un domaine connexe de l'espace-temps $V_{4}$, variété différentiable à quatre dimensions, de classe $C^{2}$ et de classe $C^{*}$ par morceaux, douée de la métrique d'univers:

$$
d s^{2}=g_{x \beta}\left(x^{\lambda}\right) d x^{\alpha} d x^{\beta}
$$

de type hyperbolique normal.

Les $g_{\alpha \beta}$ sont des fonctions de classe $C^{1}$ et de classe $C^{3}$ par morceaux.

Considérons, dans la variété espace-temps $V_{+}$, un domaine occupé par un milieu fluide. Nous dirons que l'espace-temps riemannien $V_{4}$ est associé à ce fluide.

$\vec{u}$ désignera le vecteur vitesse unitaire en chaque point du fluide. Ses trajectoires, orientées dans le temps, seront appelées lignes de conrant.

On appellera repère propre en un point du domaine de l'espace-temps, occupé par le fluide, un repère orthonormé dont le premier vecteur $\vec{V}^{(0)}$ coïncide avec le vecteur vitesse unitaire $\vec{u}$ et dont les trois autres vecteurs $\vec{V}^{(i)}$ orientés dans l'espace, sont normés par la condition $\left(\vec{\nabla}^{(t)}\right)^{2}=-1$.

Ainsi

$$
\begin{gathered}
g_{\alpha \beta} u^{\alpha} u^{\beta}=+1 \\
g_{\alpha \beta} V^{(i) \alpha} V^{(i) \beta}=-1 .
\end{gathered}
$$

Ce repère propre doit être identifié à un repère galiléen local. L'axe de temps a la direction du vecteur $\vec{u}$. L'espace associé est défini par le tri-plan $\left(\vec{V}^{(i)}\right)$.

On peut naturellement rapporter le voisinage d'un point de $V_{4}$ au repère propre en ce point.

Le repère propre qui a été introduit, est caractérisé par la matrice

$$
\left(\delta_{\alpha^{\prime} \beta^{\prime}}\right)=\left(\begin{array}{rrrr}
1 & 0 & 0 & 0 \\
0 & -1 & 0 & 0 \\
0 & 0 & -1 & 0 \\
0 & 0 & 0 & -1
\end{array}\right) .
$$

La métrique d' univers de $V_{4}$ prend la forme

$$
d s^{2}=\delta_{\alpha^{\prime} \beta^{\prime}} \cdot \dot{\omega}^{x^{\prime}} \omega^{\beta^{\prime}}=\left(\omega^{0^{\prime}}\right)^{2}-\left(\omega^{\prime \prime}\right)^{2}-\left(\omega^{2 \prime}\right)^{2}-\left(\omega^{3^{\prime}}\right)^{2} .
$$

Dans la théorie de la relativité générale, l'nnivers est regardé comme une variété dont les systèmes de référence en un point se repèrent entre 
eux comme dans la relativité restreinte: dans celle-oi l'espace-temps rapporté au repère propre admet la métrique

$$
d s^{2}=\left(d x^{0 \prime}\right)^{2}-\left(d x^{\prime \prime}\right)^{2}-\left(d x^{2}\right)^{2}-\left(d x^{3}\right)^{2}
$$

où $x^{0 \prime}=e t, c$ étant la vitesse de propagation de la lumière dans le vide. Cette remarque permet de faire l'interprétation en termes de temps et d'espace, des équations de la relativité générale.

$\mathrm{Si}$, au point considéré, l'espace-temps est rapporté à un repère quelcon. que, ce point est défini par des coordonnées curvilignes locales $x^{x}$, et la métrique d'univers a la forme $d s^{2}=g_{x \beta} d x^{\alpha} d x^{\beta}$. Les $g_{\alpha \beta}$ sont donnés en fonction des composantes des vecteurs du repère propre, par los formules:

$$
g_{\alpha \beta}=u_{\alpha} u_{\beta}-\sum_{i=1}^{3} V_{\alpha}^{(i)} V_{\beta}^{(i)}
$$

comme on a vu au $\$ 4$.

Soit $\vec{q}$ un vecteur défini au point considéré. La composante de temps de $\vec{q}$ est sa projection sur $\vec{u}$. Elle a pour composantes dans le repere naturel

$$
\vec{u} \cdot \vec{q}) u_{\alpha}=\left(u^{p} q_{p}\right) u_{\alpha}
$$

Sa composante d'espace est sa projection sur l'élément-plan normal à $\vec{u}$. Elle est definie par ses composantes en repère naturel :

$$
\stackrel{*}{q}_{\alpha}=q_{\alpha}-\left(u \rho q_{\rho}\right) u_{\alpha} \equiv q_{\rho}\left(g_{\alpha}^{\rho}-u \rho u_{\alpha}\right) \text {. }
$$

\section{II. - La conduction de la chaleur.}

\section{L'hypothèse de Fourier en relativité.}

La conduction thermique dans un milieu fluide peut être rendue compte au moyèn de l'hypothèse de FourIER. Nous nous proposons de faire une extension relativiste de cette hypothèse.

Considérons à cet effet, un domaine $D$ de l'espace-temps associé au milieu conducteur. En un point $x$ de ce domaine, la conduction thermique est liée à un vecteur $\vec{q}$ appelé vecteur courant de chaleur.

Par rapport au repère propre en ce point, ce vecteur a pour composantes

$$
\begin{gathered}
q_{0}{ }^{\prime}=0 \\
q_{i}{ }^{\prime}=-x \partial_{i} \theta
\end{gathered}
$$

où $\theta$ désigne le champ scalaire de température et $\partial_{i}$ la dérivée pfaffienne relatives aux formes $\omega^{x^{\prime}}$.

$x$ est un coefficient thermodynamique appelé conductivite thermique du milieu. Il dépend de la nature du milieu. Pour un milieu isotrope, il garde la mème valeur dans toutes les directions. Mais $x$ peut varier avec l'état $d u$ milieu, et en particulier, il varie avec la température $\theta$. Sa connaissance est demandée à l'expérience. 
Il est nécessaire de savoir que le coefficient $x$ actuel est égal au coefficient de conductivité en unités $C G S$, divisé par la vitesse limite $c$, exprimée avec les mêmes unités.

Le vecteur $q_{2,}$ est un vecteur d'espace. Ses composantes contravariantes ont pour valeurs dans le repère propre:

$$
q^{\lambda^{\prime}}=-q_{\lambda^{\prime}}
$$

Rapportons maintenant l'espace-temps an point considéré ì un repère naturel associé à un système de coordonnées curvilignes $x^{x}$. La matrice de passage d'un repère à l'autre est $\left(A^{x_{\lambda^{\prime}}}\right)$. Soit $\left(A^{\alpha^{\prime}} \lambda\right)$ la matrice inverse. Les $A^{\alpha_{\lambda^{\prime}}}$ sont les composantes contravariantes des vectenrs $V^{\left(\lambda^{\prime}\right)}$ du repère propre dans le nouveau repère. Soit

$$
\begin{array}{ll}
A_{0^{\prime}}^{\alpha}=u^{\alpha} & A_{x}^{0^{\prime}}=u_{x} \\
A_{i^{\prime}}^{\alpha}=V^{(i) \alpha} & A_{\alpha}^{i^{\prime}}=-V_{\alpha}^{(i)} .
\end{array}
$$

Dans ces formules et dans celles qui suivent, on donne la mème valeur numérique à deux indices ne différant que par l'accent'.

Nous passons des composantes $q_{\lambda^{\prime}}$ du vecteur $\vec{q}$ dans le repère propre, a ses composantes covariantes générales quelconques $q_{x}$, par les formules:

$$
q_{\alpha}=q_{\lambda^{\prime}} A_{\alpha}^{\lambda^{\prime}}=q_{i} \cdot A_{\alpha}^{i^{\prime}} \text {. }
$$

D'autre part dans le changement de rẹpères

$$
\partial_{i^{\prime}} \theta=\partial_{\rho} \theta A_{i^{\prime}}^{\rho}
$$

et par suite

$$
q_{i^{\prime}}=-x \partial_{\rho} \theta A_{i^{\prime}}^{p}
$$

En portant cette valeur de $q_{i}^{\prime}$ dans les relations précédentes, nous obtenons

$$
q_{x}=-x \partial_{p} \theta A_{i^{\prime}}^{\rho} A_{\alpha}^{i^{\prime}}=-x \partial_{\rho} \theta\left(-\sum_{i=1}^{;} V(i) \rho V_{x}^{(i)}\right)
$$

D'après la formule (7.5) du paragraphe précédent, $-\sum_{i=1}^{3} V^{(i) p} V_{x}^{(i)}=g_{x}^{\prime \prime}-u\left(i u_{\alpha}\right.$. Nous en déduisons

$$
q_{\alpha}=-x \partial_{\rho} \theta\left(g_{\alpha}^{\rho}-u \rho u_{\alpha}\right)
$$

en remarquant que les $q_{x}$ sont les composantes d'espace du vecteur $-\times \operatorname{grad} \theta$.

Le vecteur $\vec{q}$ représente le flux de chaleur à travers une surface normale à la direction $q_{z}$, par unité de surface et de temps. Dans l'espacetemps $V_{4}$, on pourrait dire que $\vec{q}$ permet de définir le flux de chaleur à travers un élément 3-plan orienté dans le temps. 
On pent donner à $q_{x}$ l'expression équivalente à (8.4)

$$
q_{\alpha}=-x\left[\partial_{x} \theta-\left(u p \partial_{\rho} \theta\right) u_{\alpha}\right]
$$

Par montée de l'indice $\alpha$, on déduit ses composantes contravariantes $q^{\alpha}$ :

$$
q^{x}=-x\left[\partial x \theta-\left(u p \partial_{\rho} \theta\right) u^{x}\right]
$$

où l'on a posé $\partial^{\alpha} \theta=g^{\alpha \lambda} \partial_{\lambda} \theta$.

En calculant le produit contracté $u^{\alpha} q_{\alpha}=u_{x} q^{x}$, on vérifie que:

$$
\boldsymbol{u}^{x} q_{x} \equiv u_{x} q^{x} \equiv \cap
$$

Cette identité appelle deux remarques:

1. $-\vec{q}$ est un vecteur purement spatial, normal an vecteur vitesse unitaire $\vec{u}$. Sa grandeur est done négative.

$$
(\vec{q})^{2}=x^{2}\left(\triangle_{1} \theta-Z^{2}\right) \leq 0 \quad\left(Z=u \rho \partial_{\rho} \theta\right) .
$$

2. - l'identité $u_{\alpha} q^{\alpha} \equiv 0$ traduit en un certain sens l'absence de toute «densité de charge calorifique».

Définimion. - On appellera lignes de chaleur, les trajectoires du champ de vecteur-courant de chaleur $\vec{q}$.

Ces lignes orientées dans l'espace, sont orthogonales aux lignes de courant. Elles intẹrviennent pour la description de certains phénomènes calorifiques.

\section{Chaleur dégagée par un élément quadridimensionnel de fluide.}

Soit un domaine $D_{4}$ de l'espace-temps, occupé par un fluide thermodynamique. Nous pouvons considérer des éléments quadridimensionnels de ce fluide enfermés dans les éléments d'hypervolume

$$
d \tau=\sqrt{|g|} d x^{0} \wedge d x^{1} \wedge d x^{2} \wedge d x^{3}
$$

de l'espace-temps associé; et nous pouvons suivre leur évolution grâce an repère naturel attaché à chaque élément. Mais, pour en dégager plus facilement le sens, nous rapportons chaque élément d'abord aux repères propres.

L'élément de fluide au point $x \in D_{4}$, est caractérisé dans le repère propre, avant la modification, par sa masse $m$, son volume ordinaire $\omega^{1 t} \wedge \omega^{2 \prime} \wedge \omega^{3 t}$ et sa température $\theta$. Dans l'étude des modifications d'état, on introduit le volume spécifique

$$
\bar{\omega}=\frac{1}{\rho}
$$

où $\rho$ est la densité propre du fluide en $x$.

Après la modification élémentaire, cet élément de fluide a un volume spécifique $\bar{\omega}+d \bar{\omega}$ et une température $\theta+d \theta$. Au cours de cette modification d'état, une quantité de chaleur $Q_{e}$ est mise en jeu et est égale a :

$$
Q_{e}=c d \theta \wedge m+l d \bar{\omega} \wedge m .
$$


Les coefficients calorifiques $c$ et $l$ s'appellent respectivement la chaleur spécifique à volume constant et la chaleur de dilatation du fluide. Ce sont, en général, des fonotions de $\bar{\theta}$ et $\theta$, ou, ce qui revient an même, de $\rho$ et $\theta$.

$$
c=c(\rho, \theta) \quad l=l(\rho, \theta) \text {. }
$$

Nous avons introduit dans (9.3) la forme différentielle extérieure $m$ ou forme élément de matière. Elle a pour composante non nulle dans le repère propre

$$
m=\rho u^{0 \prime} \omega^{\prime \prime} \wedge \omega^{2 \prime} \wedge \omega^{3 \prime} .
$$

Sous forme invariante, nous la définirons par:

$$
m=\frac{1}{3 !} \eta_{\alpha_{0}^{\prime} \alpha_{1}^{\prime} \alpha_{2}^{\prime} \alpha_{3}^{\prime} \rho} \rho u^{\alpha_{0}^{\prime} \omega^{\alpha_{1}^{\prime}}} \wedge \omega^{\alpha_{z^{\prime}}} \wedge \omega^{\alpha_{3}^{\prime}}
$$

où $\eta_{\alpha_{0}^{\prime} \alpha_{1}^{\prime} \alpha_{2^{\prime}} \alpha_{z^{\prime}}}$ est le tenseur complètement antisymétrique attaché à la forme élément de volume spatio-temporel.

$\theta$ et $\bar{\omega}$ sont des fonctions scalaires définies en $x \in D_{4}$ (formes d'ordre zéro). Nous avons:

$$
\begin{gathered}
d \theta=\partial_{\alpha^{\prime}} \theta \omega^{\alpha^{\prime}} \\
d \bar{\omega}=\partial_{\alpha^{\prime}} \bar{\omega} \omega^{\alpha^{\prime}}=-\frac{1}{\rho^{2}} \partial_{\alpha^{\prime}} \rho \omega^{\alpha^{\prime}} .
\end{gathered}
$$

Dans ces formules, $\partial_{\alpha^{\prime}}$ désigne la dérivée pfaffienne.

La quantité $Q_{e}$ a alors pour expression:

$$
Q_{e}=\left(c \partial_{\alpha^{\prime}} \theta \omega^{\alpha^{\prime}}-\frac{l}{\rho^{2}} \partial_{\alpha^{\prime}} \rho \omega^{\alpha^{\prime}}\right) \wedge \frac{1}{3 !} \eta_{x_{0}^{\prime} \alpha_{2}^{\prime} \alpha_{2}^{\prime} \alpha_{3}^{\prime} \rho} u^{\alpha_{0}^{\prime} \omega^{\alpha_{1}^{\prime}}} \wedge \omega^{\alpha_{2}^{\prime}} \wedge \omega^{\alpha_{3}^{\prime}}
$$

En effectuant les calculs, nous aurons:

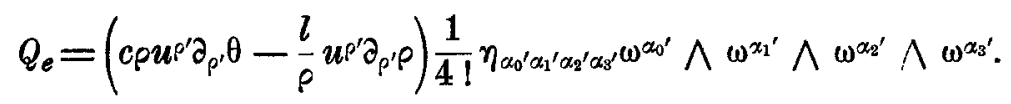

Le second facteur n'est autre que la forme élément de volume. Il vient finalement (repère propre)

$$
Q_{e}=\left(c \rho u^{0 \prime} \partial_{0^{\prime}} \theta-\frac{l}{\rho} u^{0 /} \partial_{0^{\prime} \rho}\right) \omega^{0^{\prime}} \wedge \omega^{t^{\prime}} \wedge \omega^{2 \prime} \wedge \omega^{3^{\prime}} .
$$

Si nous rapportons maintenant l'espace-temps au point considéré, au repère naturel associé à un système de coordonnées curvilignes locales $x^{\alpha}$, la formule (9.6) prend la forme invariante que l'on dédnit de (9.5)

$$
\left.Q_{e}=\left(c \rho u^{\alpha} \partial_{\alpha} \theta-\frac{l}{\rho} u^{\alpha} \partial_{\alpha \rho}\right)\right) \sqrt{|g|} d x^{0} \wedge d x^{1} \wedge d x^{2} \wedge d x^{3}
$$

C' est cette quantité que nous appelons la chaleur dégagée relative à un éloment quadridimensionnel de fluide attaché au point $\left(x^{\alpha}\right)$ quand ce point vient au point voisin $\left(x^{\alpha}+d x^{x}\right)$. 
On remarque que $u^{\star \partial_{\alpha} \theta}$ et $u \rho \partial_{\rho} \rho$ sont les projections sur $\vec{u}$ des vecteurs gräd $\theta$ et grad $\rho$ : ce sont les variations de la température $\theta$ et de la densité $\rho$ le long des lignes de courant.

\section{L'équation relativiste de la conduction thermique.}

Le vecteur courant de chaleur $\vec{q}$ qui a été défini au $\$ 8$, satisfait à une formule de divergence appelée équation de conduction. Nous allons en établir l'expression relativiste.

Considérons" un domaine $D_{4}$ de l'espace-temps occupé par un fluide thermodynamique conducteur.

Choisissons dans le domaine $D_{4}$, un volume à quatre dimensions $B_{4}$ limité par un tube de courant $S$ et par deux sections de ce tube $\Sigma_{1}$ et $\Sigma_{2}$ qui sont des hypersurfaces engendrées par les lignes de chaleur. Nous les appelons brièvement sections d'espace.

Nous supposerons dans la suite, que les différentes grandeurs introduites sont continues et à dérivées continues dans le volume $B_{4}$.

Adoptons des coordonnées telles que les lignes sur lesquelles la coor. donnée $x^{0}$ varie seule, coïncident avec les lignes de courant. De cette façon, nous pouvons suivre chaque élément de volume quadridimensionnel $d \tau$ le long de la ligne de courant $x^{i}=$ const. qui le traverse.

En intégrant $Q_{e}$ le long de cette ligne de courant, nous avons la quantité de chaleur dégagée, au sens que nous avons donné à ce mot, dans le tube d' univers infiniment mince où varie l'élément $d \tau$. En sommant ensuite sur tous les tubes d'univers infiniment minces constituant le tube envisage, et en passant à la limite, nous obtiendrons l'intégrale:

$$
Q=\iiint \int_{B_{4}}\left(c \rho u^{\times} \partial_{\alpha} \theta-\frac{l}{\rho} u^{\wedge} \partial_{x \rho}\right) \sqrt{\mid g} d x^{0} \wedge d x^{1} \wedge d x^{2} \wedge d x^{3}
$$

étendue an domaine $B_{4}$ consideré.

Par définition, la valeur $Q$ de cette intégrale est la quantité de chaleur dégagée relative au volume $B_{4}$.

Le postulat de la continuité de la chaleur consiste à affirmer que cette quantité $Q$ est égale au flux à travers la surface latérale du tube de courant, du vecteur-courant de chaleur $\vec{q}$. Grâce au choix particulier de notre domaine, elle est égale au flux de $\vec{q}$ ì travers la frontière $\partial B_{4}$ du domaine $B_{4}$, car les deux sections $\Sigma_{1}$ et $\Sigma_{2}$ étant engendrées par des lignes de chaleur, le vecteur $\vec{q}$ leur est tangent et son flux à travers $\Sigma_{1}$ et $\Sigma_{2}$ est nul.

Si $d \omega_{\alpha}$ désigne l'ólóment d'hypersurface qui limite le domaine $B_{4}$, nous avons l'égalité:

$$
\iiint \int_{B_{4}}\left(c \rho u^{\alpha} \partial_{x} \theta-\frac{l}{\rho} u^{\alpha} \partial_{x_{\varphi}}\right) \vee \overline{g \mid} d x^{0} \wedge d x^{1} \wedge d x^{2} \wedge d x^{3}=\iiint_{\partial B_{4}} q^{\alpha} d \omega_{x} .
$$


En transformant par la formule de Sтокеs, la première intégrale du flux, en intégrale de divergence, nous écrivons cette égalité sous la forme équivalente:

$$
\iiint \int_{B_{4}}\left[\nabla_{\alpha} q^{\alpha}-\left(c \rho u^{\alpha} \vec{c}_{x x} \theta-\frac{l}{\rho} u^{\alpha} \partial_{x} \rho\right)\right] \vee \overline{|g|} d x^{0} \wedge d x^{1} \wedge d x^{2} \wedge d x^{3}=0 .
$$

Cette égalité a lieu quel que soit le domaine du type considéré, c'est-à-dire limité par un tube de courant et par deux hypersurfaces engendrées par les lignes de chaleur. En vertu des hypothèses de coutinuité faites au début $\mathbf{d u}$ paragraphe, l'elément d'intégration est continu. Il en résulte qu'il doit être identiquement nul. Nous en dédnisons:

$$
\nabla_{\alpha} q^{\alpha}-\left(c \rho u^{\alpha} \partial_{\alpha} \theta-\frac{l}{\rho} u^{\alpha} \partial_{\alpha \rho}\right)=0 .
$$

L'égalité précédente est vérifiée en tout point du domaine $D_{4}$, il en résulte que l'on a encore la formule intégrale (10.3) pour tout domaine $B_{4}$ d'un type quelconque pris dans $D_{4}$.

$\mathrm{Si}$ nous supposons maintenant que le vecteur courant de chaleur est donné par l'hypothèse de Fourier, à l'aide du champ de température $\theta$, il suffit de supposer que $\theta$ est de classe $\left(C^{2}, C^{4}\right.$ par morceaux) pour que le résultat précédent soit vrai.

Nous énonçons done comme conséquence du postulat de continuité de la chaleur le théorème:

* ThÉORÈme. - Soit un domaine $D_{4}$ de l'espace-temps, occupé par un fluide thermodynamique conducteur. Pourvu que $\theta$ soit une fonction de classe $\left(C^{2}, C^{*}\right.$ par morceaux), et que les quantités $u^{2}, \rho, x$, soient continues ainsi que leurs dérivées, alors le vecieur courant de chaleur $q^{\alpha}$ du fuide satisfait en chaque point $d u$ domaine $D_{4}$, à l'équation:

$$
\nabla_{x} q^{\alpha}=c \rho u^{x} \partial_{x} \theta-\frac{l}{\rho} u^{\alpha} \partial_{x \rho}
$$

et pour tout volume à quatre dimensions $B_{4}$, pris dans $D_{4}$, ̀̀ l'égalité intégrale:

$$
\iiint \int_{B_{4}}\left(c \rho u^{\alpha} \partial_{\alpha} \theta-\frac{l}{\rho} u^{\alpha} \partial_{\alpha} \rho\right) \sqrt{|g|} d x^{0} \wedge d x^{1} \wedge d x^{2} \wedge d x^{3}=\iiint_{\partial B_{4}} q^{\alpha} d \omega_{\alpha}
$$

où $\partial B_{4}$ désigne le bord de $B_{4}$.

C' est l'égalité (10.5) qui traduit finalement le postulat de la continuité de la chaleur. L'équation anx dérivées partielles (10.4) que nons appellerons équation de conduction thermique, généralise une equation classique de FoURIER. 


\section{III. - Le tenseur d'impulsion-énergie et les équations du fuide thermodynamique relativiste.}

11. Le tenseur d'impulsion-énergie du schéma fluide thermodynamique.

Considérons un domaine $D_{4}$ de l'espace-temps occupé par un milieu fluide thermodynamique tel qu'il a été défini dans les sections précédentes.

Nous nous proposons d'établir les équations du fluide thermodynamique. Or, nous connaissons les raisons qui ont conduit au système des équations d' EINSTEIN :

$$
S_{x \beta}=\chi T_{x \beta} \text {. }
$$

Le tenseur $S_{\alpha \beta}$ ne dépend que de la structure de variété riemannienne de l'espace-temps. Il est conservatif, e' est-à-dire qu'il satisfait aux quatre conditions $\nabla_{\alpha} S^{\alpha \beta}=0$. Le tenseur $T_{\alpha \beta}$ satisfait alors aussi aux quatre conditions :

$$
\nabla_{\alpha} T^{\alpha \beta}=0 .
$$

Comme il est de signification purement physique, il doit donner la description de la matière et de son mouvement. Nous postulons done que les conditions de conservation sont l'expression des équations de mouvement du fluide thermodynamique.

Notre problème consiste à choisir le tenseur d'impulsion-énergie $T^{\alpha \beta}$. Il doit décrire au mieux les propriétés physiques du milieu. Et les grandeurs qui figurent dans ce tenseur seront les éléments du modèle plus ou moins simplifié, $d u$ concept approprié au problème traité, par lequel nous remplaçons la matière réelle.

Le traits reconnus expérimentalement comme les plus importants doivent évidemment être introduits et placés par ordre d'importance, dans notre mode de description. Les éléments de cette description sont cenx que la mécanique ef la thermodynamique appliquent aux théories de l'hydrodynamique et de l'élasticité. Il est done indiqué de faire figurer dans l'expression du tenseur d'énergie du fluide thermodynamique:

1. - un terme principal de beaucoup le plus important qui sera le tenseur d'énęrgie de la matière et de l'énergie pondérable.

2. - les pressions propres.

3. - les échanges thermiques consistant, au sein du milieu, en échanges par conduction.

4. - le champ électromagnétique.

Nous serons ainsi conduits à un schéma simple représenté par le tenseur d' impulsion-énergie suivant:

$$
T^{\alpha \beta}=\rho u^{\alpha} u^{\beta}-\pi^{\alpha \beta}-Q^{\alpha \beta}+\tau^{\alpha \beta}
$$

où les termes successifs sont ceux qui ont été énoncés. 
Le schéma sera dit schéma "fluide thermodynamique pur» ou simplement "schéma thermodynamique", si le terme dû au champ électromagnétique est négligé. C' est un tel schéma que nous nous proposons d'étudier.

Nous rapportons d'abord le fluide au voisinage d'un point $x$ de $D_{4}$, au repère propre en ce point: dans ce repère la matière est au repos au point envisagé. Le fluide $y$ est caractérisé par une densité propre $\rho$, le tenseur iles pressions propres $\pi^{i^{\prime} k^{\prime}}$ et le vecteur courant de chaleur $q_{i}^{\prime}$.

12. Fxpression des composantes du tenseur d'énergie.

Si nous adoptons le tenseur d'expression générale

$$
T^{\alpha \beta}=\rho u^{\alpha} u^{\beta}-\pi^{\alpha \beta}-Q^{\alpha \beta}
$$

pour tenseur d'impulsion-énergie $d$ ' un schéma thermodynamique, les raisons qui viennent d'être exposées, nous conduisent à admettre que le tenseur d'impulsion-énergie doit avoir dans le repère propre, comme valeurs des composantes, les valeurs suivantes:

$$
T^{0,0^{\prime}}=\rho \quad T^{0^{\prime \prime} \prime}=T^{i 0^{\prime}}=-q^{i^{\prime}} \quad T^{i^{\prime} k^{\prime}}=-\pi^{i / k^{\prime}} .
$$

Ce sont ces valeurs qui ont été adoptées dans notre théorie.

Elles sont justifiées par les conséquences.

Pour avoir l'expression invariante générale des composantes du tenseur d'énergie $T^{\alpha \beta}$, il suffit de faire un changement de repère.

Rapportons donc l'espace-temps, au voisinage du point considéré, au repère naturel, associé à un système de coordonnées curvilignes locales quelconque $x^{\alpha}$. La matrice de passage $d^{\prime}$ un repère à l'autre est $\left(A_{x^{\prime}}^{\lambda}\right)$.

Nous passons des composantes $T^{\lambda^{\prime} \mu^{\prime}}$ du tenseur d'énergie dans le repère propre $\left.\vec{u}, \overrightarrow{v^{\prime}} i^{\prime}\right)$ à ses composantes contravariantes générales, par les formules de transformation

$$
T^{\alpha \beta}=T^{\lambda \mu^{\prime}} A_{\lambda^{\prime}}^{\alpha} A_{\mu^{\prime}}^{\beta} .
$$

En développant les calculs, nous avons

$$
T^{\alpha \beta}=T^{0^{\prime} 0^{\prime}} A_{0^{\prime}}^{\alpha} A_{0^{\prime}}^{\beta}+T^{i^{\prime} k^{\prime}} A_{i^{\prime}}^{\alpha} A_{k^{\prime}}^{\beta}+T^{0^{\prime} i^{\prime}} A_{0^{\prime}}^{\alpha} A_{i^{\prime}}^{\beta}+T^{i 0^{\prime}} A_{i^{\prime}}^{\alpha} A_{0^{\prime}}^{\beta} .
$$

Nous avons à $y$ substituer les valeurs de $T^{\lambda^{\prime} \mu^{\prime}}$ données par les formules (12.2) et les valeurs de $A_{\lambda^{\prime}}^{x}$, en fonction des composantes des vecteurs du repère propre (cf. formules (8.3) $\$ 8$ ). Nous obtenons:

$$
T^{\alpha \beta}=\rho u^{\alpha} u^{\beta}-\sum_{i^{\prime}, k^{\prime}} \pi^{i^{\prime} k^{\prime}} V^{\left(i^{\prime}\right) \alpha} V^{\left(k^{\prime}\right) \beta}-\sum_{i^{\prime}} q^{i^{\prime}} u^{\alpha} V^{(i) \beta}-\sum_{i^{\prime}} q^{i^{\prime}} V^{\left(i^{\prime}\right) x} u^{\beta} .
$$

Nous poserons

$$
\begin{gathered}
\pi^{\alpha \beta}=\sum_{i^{\prime}, k^{\prime}} \pi^{i^{\prime} k^{\prime}} V^{\left(i^{\prime}\right) \alpha} V^{\left(k^{\prime}\right) \beta} \\
q^{\alpha}=\sum_{i^{\prime}}^{\Sigma} q^{i^{\prime}} V^{\left(i^{\prime}\right) \alpha} \\
Q^{\alpha \beta}=u^{\alpha} q^{\beta}+u^{\beta} q^{\alpha} .
\end{gathered}
$$


Dans le cas de l'hypothèse de Fourier concernant la conduction de chaleur, nous pouvons vérifier que le vecteur $q^{\alpha}$ qui figure dans le tenseur d'énergie $T^{\alpha \beta}$. a bien l'expression relativiste établie au $\S 8$. En effet, si nous admettons dans le repère propre

$$
q_{i^{\prime}}=-x \partial_{i^{\prime}} \theta
$$

nous avons $q^{i}=-q_{i^{\prime}}=x \partial_{i^{\prime}} \theta$. Après le changement de repère, nous obtenons :

$$
\sum_{i^{\prime}}^{\Sigma} q^{i^{\prime}} V^{\left(i^{\prime}\right) x}=\sum_{i^{\prime}} x \partial_{i^{\prime}} \theta V^{\left(i^{\prime}\right) x}=\sum_{i^{\prime}} x \partial_{\rho} \theta A_{i^{\prime}}^{P^{\prime}} V^{\left(i^{\prime}\right) x} \text {. }
$$

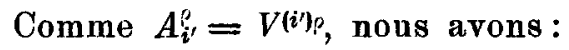

$$
q^{\alpha}=\sum_{i^{\prime}}^{\Sigma} q^{i^{\prime}} V^{\left(i^{\prime}\right) \alpha}=x \partial_{\rho} \theta \underset{i^{\prime}}{\Sigma} V^{\left(i^{\prime}\right) \rho} V^{\left(i^{\prime}\right) \alpha}=-x \partial_{\rho} \theta\left(g^{\rho x} \ldots u \rho u^{x}\right) .
$$

Dans le cas général, le raisonnement que nous avons fait, nous conduit i adopter pour le schéma fluide-thermodynamique, l'expression générale suivante du tenseur d'impulsion-énergie :

$$
T^{\alpha \beta}=\rho u^{\alpha} u^{\beta}-\pi^{\alpha \beta}-\left(u^{\alpha} q^{\beta}+u^{\beta} q^{\alpha}\right) .
$$

La partie $Q^{\alpha \beta}=u^{\alpha} q^{\beta}+u^{\beta} q^{x}$ correspond aux échanges thermiques par conductibilité dans le milieu.

Dans le repère propre:

$$
\begin{aligned}
\pi^{\lambda^{\prime} \mu^{\prime}} u_{\lambda^{\prime}} & =0 \\
u_{\lambda^{\prime}} q^{\lambda^{\prime}} & =0 .
\end{aligned}
$$

Les quantités $\pi^{\lambda^{\prime} \mu^{\prime}}, q^{\lambda^{\prime}}, u_{\lambda^{\prime}}$ sont des tenseurs et vecteurs; il en résulte que dans un repère arbitraire on a encore :

$$
\begin{aligned}
\pi^{\alpha \beta} \boldsymbol{u}_{\alpha} & =0 \\
\boldsymbol{u}_{\alpha} \boldsymbol{q}^{\alpha} & =0
\end{aligned}
$$

On peut vérifier ce résultat sur les formules (12.5).

13. Les équations de mouvement pour un schéma thermodynamique.

Considérons un schéma fluide thermodynamique où la distribution énergétique dans l'espace-temps associé, est représentée par le tenseur:

$$
T^{\alpha \beta}=\rho u^{\alpha} u^{\beta}-\pi^{\alpha \beta}-\left(u^{\alpha} q^{\beta}+u^{\beta} q^{\alpha}\right) .
$$

Les équations de mouvement $d u$ fluide thermodynamique considéré, sont fournies par les conditions de conservation (cf. \& 11):

$$
\nabla_{\alpha} T^{\alpha \beta}=0
$$

appliquées à la forme (13.1) de son tenseur d'impulsion-energie.

Nous avons, en prenant la dérivée covariante de $T^{\alpha \beta}$

$$
\rho \boldsymbol{u}^{\alpha} \nabla_{\alpha} \boldsymbol{u}^{\beta}+\boldsymbol{u}^{\beta} \nabla_{\alpha}\left(\boldsymbol{\rho} \boldsymbol{u}^{\alpha}\right)-\nabla_{\alpha} \pi^{\alpha \beta}-\nabla_{\alpha}\left(\boldsymbol{u}^{\alpha} q^{\beta}+\boldsymbol{u}^{\beta} q^{\alpha}\right)=0 .
$$


Le vecteur $\vec{u}$ est unitaire et orienté dans le temps:

$$
g_{\alpha \beta} u^{\alpha} u^{\beta}=1 \text {. }
$$

En différentiant, on en déduit:

$$
u_{\beta} \nabla_{a} u^{\beta}=0 \text {. }
$$

D'autre part, le vecteur courant de chaleur est orthogonal au vecteur vitesse unitaire $\vec{u}$ :

$$
u_{\beta} q^{\beta}=0 \text {. }
$$

Par multiplication contractée de (13.2) par $u_{\beta}$, il vient, en tenant compte des relations précédentes :

$$
\nabla_{\alpha}\left(\rho u^{\alpha}\right)-u_{\beta} \nabla_{\alpha} \pi^{\alpha \beta}-\nabla_{\alpha} q^{\alpha}+q^{\rho} u^{\alpha} \nabla_{\alpha} u_{\rho}=0 .
$$

Nous portons ensuite la valeur de $\nabla_{x}\left(\rho u^{\alpha}\right)$ tirée de cette équation, dans (13.2). Nous obtenons l'équation :

$$
\rho u^{\alpha} \nabla_{\alpha} u^{\beta}+u^{\beta}\left(u_{\rho} \nabla_{a} \pi^{\alpha \rho}+\nabla_{\alpha} q^{\alpha}-q^{\rho} u^{\alpha} \nabla_{\alpha} u_{\rho}\right)-\nabla_{\alpha^{\prime}} \pi^{\alpha}-\nabla_{\alpha}\left(u^{\alpha} q^{\beta}+u^{\beta} q^{\alpha}\right)=0
$$

que nous pouvons écrire:

$$
\rho u^{\alpha} \nabla_{\alpha} u^{\beta}-\nabla_{\alpha} \pi^{\alpha \rho}\left(g_{\rho}^{\beta}-u_{\rho} u^{\beta}\right)-\nabla_{\alpha}\left(u^{\alpha} q^{\beta}+u^{\beta} q^{\alpha}\right)+u^{\beta}\left(\nabla_{\alpha} q^{\alpha}-q^{\rho} u^{\alpha} \nabla_{\alpha} u_{\rho}\right)=0 .
$$

Nous mettrons finalement les équations (13.3) et (13.4) sous la forme:

$$
\begin{gathered}
\nabla_{\alpha}\left(\rho u^{\alpha}\right)-u_{\beta} \nabla_{x^{2}} \pi^{\alpha \beta}=\nabla_{\alpha} q^{\alpha}-q^{\beta} u^{\alpha} \nabla_{\alpha} u_{\beta} \\
\rho u^{\alpha} \nabla_{x} u^{\beta}-\nabla_{\alpha} \pi^{\alpha \rho}\left(g_{\rho}^{\beta}-u_{p} u^{\beta}\right)=\nabla_{x}\left(u^{\alpha} q^{\beta}+u^{\beta} q^{\alpha}\right)-u^{\beta}\left(\nabla_{\alpha} q^{\alpha}-q^{\rho} u^{\alpha} \nabla_{\alpha} u_{p}\right) .
\end{gathered}
$$

L'équation (13.5) joue le ròle d' une équation de continuité pour le milieu. L'énergie d'origine calorifique y figure effectivement. Nous verrons au chapitre suivant, qu' elle est lourde de signification thermodynamique.

D'autre part, les lignes de courant du schéma considéré sont définies com. me lignes tangentes au vecteur vitesse unitaire $\vec{n}$, e' est-à-dire les trajectoires du champ de vecteur $\vec{u}$. Les équations (13.6) où l'on posera:

$$
u^{\alpha}=\frac{d x^{\alpha}}{d s}
$$

constituent un système différentiel qui déterminera les lignes de courant du schéma.

Il convient d'ajouter aux équations précédentes, les relations supplémentaires qui définissent le vecteur courant de chaleur $q^{\alpha}$

$$
\begin{gathered}
q^{\alpha}=-x \partial_{\rho} \theta\left(g^{\rho \alpha}-u \rho u^{\alpha}\right) \\
\nabla_{x} q^{\alpha}=c \rho u^{\alpha} \partial_{\alpha} \theta-\frac{l}{\rho} u^{\alpha} \partial_{\alpha} \rho .
\end{gathered}
$$

Elles détermineront les lignes de chaleur et le champ de température du schéma. 
14. Cas d'un fluide parfait thermodynamique.

Un fluide est dit parfait si la résultante des forces superficielles $\vec{T} d S$, agissant sur un élément de surface orientée $d S$, est normale à cet élément. Dans ce cas, la quadrique des pressions, définie dans la repère propre par la forme quadratique de coefficients $\pi_{i^{\prime} k^{\prime}}$, est une sphère.

Le tenseur des pressions dans le repère propre a pour expression:

$$
\pi_{i^{\prime} k^{\prime}}=p \delta_{i^{\prime} k^{\prime}}
$$

où $p$ est la pression scalaire du fluide au point considéré.

Les formules de transformation du $\$ 12$ conduisent à l'expression invariante générale dans l'espace-temps $V_{4}$ :

$$
\pi^{\alpha \beta}=\sum_{i / k^{\prime}} p \delta_{i^{\prime} k^{\prime}} V^{\left(i^{\prime}\right) \alpha} V^{\left(k^{\prime}\right) \beta}=p \underset{i^{\prime}}{\Sigma}-V^{\left(i^{\prime}\right) \alpha} V^{\left(i^{\prime}\right) \beta}
$$

d'où

$$
\pi^{\alpha \beta}=p\left(g^{\alpha \beta}-u^{\alpha} u^{\beta}\right) .
$$

Nous donnerons à l'expression du tenseur d'impulsion-énergie du schéma fluide parfait thermodynamique, la forme suivante:

$$
T^{\alpha \beta}=(\rho+p) u^{\alpha} u^{\beta}-p g^{\alpha \beta}-\left(u^{\alpha} q^{\beta}+u^{\beta} q^{\alpha}\right):
$$

Les équations de mouvement pour un schéma fluide parfait thermody. namique, prennent la forme:

$$
\begin{gathered}
\nabla_{\alpha}\left[(\rho+p) u^{\alpha}\right]-u^{\alpha} \partial_{\alpha} p=\nabla_{\alpha} q^{\alpha}-q^{\beta} u^{\alpha} \nabla_{a} u_{\beta} \\
(\rho+p) u^{\alpha} \nabla_{\alpha} u^{\beta}-\partial_{\alpha} p\left(g^{\alpha \beta}-u^{\alpha} u^{\beta}\right)=\nabla_{\alpha}\left(u^{\alpha} q^{\beta}+u^{\beta} q^{\alpha}\right)-u^{\beta}\left(\nabla_{\alpha} q^{\alpha}-q^{\beta} u^{\alpha} \nabla_{\alpha} u_{\rho}\right) .
\end{gathered}
$$

Le vecteur courant de chaleur satisfait toujours aux relations de définition (13.7) et (13.8).

Les trois quantités $\rho, p, \theta$ sont liées par une relation appelée équation d'état du fluide parfait. Cette équation d'état sera introduite sous la forme

$$
\rho=\varphi(p, \theta) \text {. }
$$

La fonction $\varphi(p, \theta)$ est demandée à l'expérience directe.

Nous étudierons au chapitre IV, comment avec ces équations se pose le problème de CaUCHY pour un fluide thermodynamique relativiste. 
CHAPITRE III.

\section{LES PRINCIPES GENERAUX}

D' UNE THERMODYNAMIQUE RELATIVISTE

\section{I. - Forme différentielle des principes de thermodynamique classique pour un fluide en mouvement.}

15. Evolution des caractéristiques d'un fluide en mouvement.

Considérons un milieu fluide en mouvement dans un domaine $D$ de l'espace. Et proposons-nous d'étudier son évolution dans l'espace et le temps.

Pour cela, nous rapportons l' espace à un système de coordonnées curvilignes quelconques $x^{1}, x^{2}, x^{3}$, en le douant de la métrique définie positive

$$
d s^{2}=g_{i j}\left(x^{k}\right) d x^{i} d x^{j}
$$

Nous désignerons par $t$ la variable temps. Et nous supposons que l'on a pu définir pour le fluide considéré, en chaque point $M\left(x^{1}\right)$ et à chaque instant $t$ :

1. - une densité de matière $\mu$.

2. - un vecteur vitesse $\vec{v}$.

Nous appellerons caractéristiques du milieu fluide, les quantités géométriques ou physiques qui penvent servir à décrire son évolution. Telles sont la densité de matière $\mu$, la vitesse $\vec{v}$, le tenseur des pressions $\pi^{i k}$, la tempéra. ture $\theta$, les densités d'energie, la densité d'entropie...

Il est clair que l'évolution du fluide peut etre étudiée gràce à l'étude de ses caractéristiques.

Or, si nous considérons une caractéristique locale du milieu continu, pour l'étudier, il nous faut la rapporter an système des quatre variables $\left(x^{1}, x^{2}, x^{3}, t\right)$. Ces variables seront dites les variables $d^{\prime}$ Euler. Si c' est une caractéristique scalaire $w$, son evolution peut ètre décrite par sa dérivée par rapport au temps

$$
\frac{d w}{d t}=\frac{\partial w}{\partial t}+\frac{\partial w}{\partial x^{1}} \frac{d x^{1}}{d t}+\frac{\partial w}{\partial x^{2}} \frac{d x^{2}}{d t}+\frac{\partial w}{\partial x^{3}} \frac{d x^{3}}{d t} .
$$

En introduisant les composantes contravariantes de la vitesse $\vec{v}$

$$
v^{i}=\frac{d x^{i}}{d t}
$$

et la notation habituelle des dérivées partielles $\partial_{i}=\frac{\partial}{\partial x^{i}}$ nous pouvons écrire

$$
\frac{d w}{d t}=\frac{\partial w}{\partial t}+v^{i} \partial_{i} w .
$$


L'évolution d'une caractéristique scalaire peut ainsi être décrite par l'opérateur de dérivation totale par rapport au temps

$$
\frac{d}{d t}=\frac{\partial}{\partial t}+v^{\prime} \partial_{i}
$$

Si la caractéristique considérée est une composante d'un vecteur ou d'un tenseur défini dans l'espace, il nous faudra remplacer le symbole de dérivation partielle $\partial_{i}$ par celui de dérivation covariante $\nabla_{i}$ relative à la métrique $d s^{2}=g_{i j} d x^{i} d x^{j}$ de l'espace.

En convenant que $\nabla_{i} w=\partial_{i} w$, si $w$ est une fonction scalaire, nous pouvons dire que l'évolution d'une caractéristique quelconque du fluide peut etre décrite par l'opérateur :

$$
\frac{d}{d t}=\frac{\partial}{\partial t}+v^{t} \nabla_{1}
$$

Avec cette notation, il est facile de donner aux équations générales de la dynamique des milieux continus $\left({ }^{2}\right)$ la forme:

$$
\begin{gathered}
\frac{d \mu}{d t}+\mu \nabla_{i} v^{i}=0 \\
\mu \frac{d v^{i}}{d t}-f^{i}+\nabla_{k} \pi^{i k}=0 .
\end{gathered}
$$

La première équation est l'équation de continuité du milieu. Les trois équations suivantes sont les équations de mouvement. $f^{i}$ sont les composantes de la résultante des forces de masse par unité de volume. $\pi^{i k}$ représente le tenseur des pressions internes.

\section{Variables de Lagrange.}

Considérons dans le domaine $D$ de l'espace, nne région $V_{0}$ occupée par un volume quelconque de fluide $a$ un instant $t=0$ pris pour instant initial. Soit $V$ la région occupée par le mème volume de fluide à l'instant $t$.

Suivons un elément de fluide dans son mouvement.

A l' instant initial $t=0$, il occupe la position $\left(a^{1}, a^{2}, a^{3}\right)$ dans $V_{0}$. A l'instant $t$ il occupe la position $\left(x^{1}, x^{2}, x^{3}\right)$ dans $V$. Le mouvement de cet élément de fluide sera défini si nous connaissons à chaque instant $t$ les trois fonctions :

$$
\begin{aligned}
& x^{1}=x^{1}\left(a^{1}, a^{2}, a^{3}, t\right) \\
& x^{2}=x^{2}\left(a^{1}, a^{2}, a^{3}, t\right) \\
& x^{3}=x^{3}\left(a^{1}, a^{2}, a^{3}, t\right)
\end{aligned}
$$

des quatres variables $a^{1}, a^{2}, a^{3}, t$.

(2) Voir Lichnfrowicz, Elements de Calcul tensoriel, chapitre IV, section III. 
L'ensemble des quatres variables $\left(a^{1}, a^{2}, a^{3}, t\right)$ sera dit les variabtes de Lagrange. Nous pouvons, a l'aide des formules (16.1) exprimer les valeurs des caractéristiques du fluide, en fonction des variables de LAGRANGE, et étudier leur évolution à l'aide de ces variables.

Les équations (16.1) definissent une transformation ponetuelle dans $D$. Nous supposerons que les $x^{i}$ sont des fonctions de classe $C^{2}$, a jacobien non nul, des variables $a^{4}, a^{2}, a^{3}, t$. Le déterminant fonctionnel $\Delta$ de la transformation est alors une fonction continue des variables $a^{1}, a^{2}, a^{3}$, $t$. Comme les $x^{i}$ coìncident avec les $a^{i}$, à l'instant initial $t=0$, il a à cet instant, la valeur +1 : la valeur de $\Delta$ est done strictement positive a tout instant.

Nous faisons des hypothèses analogues sur la transformation inverse de (16.1)

$$
\begin{aligned}
& a^{1}=a^{1}\left(x^{1}, x^{2}, x^{3}, t\right) \\
& a^{2}=a^{2}\left(x^{1}, x^{2}, x^{3}, t\right) \\
& a^{3}=a^{3}\left(x^{1}, x^{2}, x^{3}, t\right) .
\end{aligned}
$$

Dh́finimions. - Les hypothèses de continuité sur les $x^{i}$ et les $a^{i}$ entrẫnent plusieurs conséquences.

Considérons dans la région $V_{0}$ occupée par un volume de fluide à l'instant initial, des éléments formant une ligne $L_{0}$, définie par les expressions de $a^{1}, a^{2}, a^{3}$ en fonctions continues d' un paramètre $\lambda$. Le mêmes éléments à l'instant $t$ se trouveront sur la ligne continue $L$ définie par les équations (16.1) où l'on remplace $a^{1}, a^{2}, a^{3}$ par leurs expressions en fonction de $\lambda$.

Nous appelons cette ligne d'éléments, suivis ainsi dans le mouvement du fluide, une ligne fuide.

De même si l'on considère dans la région $V_{0}$ des éléments formant une surface $\Sigma_{0}$ définie par les expressions de $a^{1}, a^{2}, a^{3}$ en fonction de deux paramètres, les mêmes éléments à l'instant $t$ se trouveront sur la surface $\Sigma$ définie par les équations (16.1) où l'on remplace $a^{1}, a^{2}, a^{3}$ par leurs expres. sions en fonction de ces deux paramètres. Les éléments considérés forment dans le mouvement ce que nous appelons une surface fluide.

Si la surface $\Sigma_{0}$ est fermée, la surface $\Sigma$ est aussi fermée. Les éléments de fluide se trouvant a l'instant initial à l'intérieur de la surface fermée $\Sigma_{0}$, ne peuvent pas traverser la surface $\Sigma$. Se déplaçant avec continuité, ils restent constamment à l'intérieur de cette surface: ils forment ce que nons appelons un volume fuide.

Ces considerations nous seront fort utiles dans la suite. 
17. - Lemme.

EnoncÉ. - Considérons dans le domaine $D$ de l'espace, occupé par un fluide en mouvement, l'intégrale:

$$
J=\iiint_{V} \mu \Phi d V
$$

étendu à un volume fluide $V$ enfermé dans une surface fluide fermée $\Sigma$, oì $\mu$ est la densité du fluide au point $x^{i} \in D, \Phi$ une fonction scalaire définie au même point $x^{i}$, 'ét $d V$ l'élément de volume entourant ce point.

$S i \mu$ et $\Phi$ sont des fonctions continues des variables $x^{i}$, $t$, et admettent des dérivées partielles du ler ordre continues dans le domaine $D$, alors pour tout volume fluide $V$ intérieur au domaine $D$, on a l'égalité:

$$
\frac{d}{d t} \iiint_{V} \mu \Phi d V=\iiint_{V} \mu \frac{d \Phi}{d t} d V .
$$

Démonstration. - Dans ce qui suit, l'indice 0 affecté à une quantité, indique que cette quantité doit être prise avec sa valeur à l'instant initial $t=0$, tandis que la même quantité écrite sans indice, est prise avec sa valeur à l'instant $t$.

Soit $V_{0}$ le volume fluide à l' instant $t=0$.

L'hypothèse de continuité pour le milieu fluide, se traduit par l'égalité:

$$
\iiint_{V} \mu d V=\iiint_{V_{0}} \mu_{0} d V_{0} \text {. }
$$

Si nous effectuons le changement de variables (16.1) pour la première intégrale, elle devient $\iiint_{V_{0}} \mu_{0} \triangle d V_{0}$. L' égalité précédente conduit à

$$
\iiint_{V_{0}}\left(\mu \Delta-\mu_{0}\right) d V_{0}=0
$$

Cette égalité doit avoir lien pour tout volume $V_{0}$; en vertu des hypothèses de continuité, nous en déduisons:

$$
\mu \Delta-\mu_{0}=0
$$

ce qui est une des formes de l'équation de continuité.

Ceci étant rappelé, appliquons la transformation (16.1) à l'intégrale $J$. Elle devient :

$$
J=\iiint_{V} \mu \Phi d v=\iiint_{V_{0}} \mu \Phi \Delta d V_{0} .
$$

D'après (17.3), $\mu \Delta=\mu_{0}$, nous avons :

$$
J=\iiint_{V_{0}} \mu_{0} \Phi d V_{v}
$$


L' espace et le temps forment un continum, $D$ est un continu.

Les fonctions $\mu$ et $\Phi$, continues dans $D$, sont uniformément continues par rapport à $a^{i}$ et $t$. D'autre part elles ont des dérivées continues dans $D$, par hypothèse. Nous pouvons alors différentier la fonction $J(t)$ sous le signe d' intégration :

$$
\frac{d J}{d t}=\iiint_{V_{0}} \mu_{0} \frac{d \Phi}{d t} d V_{0} .
$$

A cette intégrale, nous appliquons la transformation (16.2) qui est la transformation inverse de (16.1). La théorie du changement de variables dans l'integration donne:

$$
\iiint_{V_{0}} \mu_{0} \frac{d \Phi}{d t} d V_{0}=\iiint_{V} \frac{\mu_{0}}{\triangle} \frac{d \Phi}{d t} d V
$$

En vertu de (17.3), $\frac{\mu_{0}}{\Delta}=\mu$. Nous en déduisons la formule du lemme:

$$
\frac{d}{d t} \iiint_{V} \mu \Phi d V=\iiint_{V} \mu \frac{d \Phi}{d t} d V .
$$

18. - Enoncés des principes de la thermodynamique appliquée à un milieu fluide.

En thermodynamique, on considère un ensemble de corps ou système, qui subit des transformations. A chaque instant, le système est dans un certain état qui est défini par les valeurs numériques d'un certain nombre de variables. Souvent, on considère ce qui s'est produit lorsque le système a passé d'un certain état initial à un certain état final. Le plus souvent, ces deux états sont des états d'équilibre. Les états intermédiaires ne sont pas des états d'équilibre. Très exceptionnellement, ces ètats intermédiaires penvent être tous infiniment voisins des états d'équilibre. On dit alors que la transformation s'effectue d'une manière réversible.

Etant donnée une transformation subie par un système, il y a lieu d'examiner non seulement ce qui s'est passé à l'intérieur du système, mais aussi les conséquences de cette transformation sur ce qui est extérieur au système. En somme, on partage l'univers en deux parties: l'une est le système dont on étudie les transformations, l'autre est tout ce qui l'entoure.

Soit maintenant un volume fluide $V$ qui évolue à l'intérieur du domaine $D$ de l'espace occupé par un fluide en mouvement.

En vertu de l' hypothèse de continuité, il n' échange que du travail et de la chaleur avec le reste du fluide. On peut le considérer comme un système au sens de la thermodynamique générale. 
Supposons que le volume fluide $V$ évolue d'un état à un état voisin correspondant aux instants $t$ et $t+d t$. Si $U$ désigne son énergie totale, le premier principe dit que

$$
d U=Q_{e}-\mathcal{G}_{e}
$$

où $Q_{e}$ et $\sigma_{e}$ sont respectivement la quantité de chaleur et la quantité de travail que le volume fluide $V$ echange avec le reste du fluide.

L'energie totale $U$ est définie comme la somme de l'énergie cinétique et d' une énergie appelée énergie interne. Si $\frac{1}{2} \mu V^{2}$ et $\mu \varepsilon$ désignent la densité d'énergie cinétique et la densité d'énergie interne, $U$ a pour valeur:

$$
U=\iiint_{V} \frac{1}{2} \mu V^{2} d V+\iiint_{V} \mu \varepsilon d V
$$

$\varepsilon$ est en général une fonction des variables d'état interne du fluide. Elle est supposée continûment différentiable.

Le volume fluide $V$ est limité par la surface fluide $\Sigma$. A travers chaque élément $d \Sigma$ de la surface $\Sigma$, il y a échange de chaleur. Nous pouvons dire qu'associé à chaque élément $d \Sigma$, le reste du fluide constitue autant de sources de chaleur vis-à-vis du système constitué par $V$. L'évolution de $V$ peut être caractérisé par le second principe.

Si nous considérons deux états voisins du volume fluide $V$ correspondant aux instants $t$ et $t+d t$, le second principe postule qu'il existe une fonction d'état $S$ appelée entropie de $V$, telle que:

$$
\frac{d S}{d t} \geq \iint_{\Sigma} \frac{q^{i} d \sigma_{i}}{\theta}
$$

$d \sigma_{i}$ désigne les composantes $d \mathfrak{u}$ vecteur représentant l'élément d'aire $d \Sigma$ de $\Sigma, \theta$ la température au centre de $d \Sigma$. $q^{i}$ est le vecteur courant de chaleur: $\frac{q^{i} d \sigma_{i}}{\theta}$ est donc la chaleur échangée sous la température $\theta$ entre $V$ et la source fictive correspondante.

L'égalité, est atteinte par une transformation réversible faisant passer le volume fluide $V$ de l'état $t$ à l'état $t+d t$.

\section{Forme différentielle du premier principe.}

Nous considérons un volume fluide $V$ limité par une surface fermée régulière $\Sigma$, de façon que le théorème de GrEke s'applique. La normale de $\Sigma$ sera orientée vers l'extérieur.

Nous supposerons dans la suite que les diverses quantités introduites sont continues ainsi que leurs dérivées. 
Les échanges de chaleur se font par conduction, les échanges par con. vection n'ont pas lieu grace au choix du volume $V$. Soit $q^{i}$ le vectenr courant de chaleur. Aux infiniment petits d'ordre supérieur près, nous avons:

$$
\begin{gathered}
Q_{e}=d t \iint_{\Sigma} q^{i} d \sigma_{i} \\
\widetilde{\sigma}_{e}=-d t\left(\iiint_{V} f^{i} v_{i} d V-\iint_{\Sigma} v_{i} \pi^{i k} d \sigma_{k}\right) .
\end{gathered}
$$

D'autre part de (18.2), nous tirons:

$$
d U=d t\left(\frac{d}{d t} \iiint_{V}^{1} \int_{2} \mu v^{2} d V+\frac{d}{d t} \iiint_{V} \mu \varepsilon d V\right)
$$

En substituant ces quantités dans (18.1), et tenant compte du lemme (17.2), nons arrivons à la relation suivante qui est vraie à chaque instant $t$ :

$$
\iiint_{V} \mu v_{i} \frac{d v^{i}}{d t} d V+\iiint_{V} \mu \frac{d \varepsilon}{d t} d V=\iint_{\Sigma} q^{i} d \sigma_{i}+\iiint_{V} f^{i} v_{i} d V-\iint_{\Sigma} v_{i} \pi^{i k} d \sigma_{k} .
$$

Arrangeons les termes de la manière suivante:

$$
\iiint_{V} \mu \frac{d \varepsilon}{d t} d V=\iint_{\Sigma} q^{i} d \sigma_{i}-\iiint_{V} \mu v_{i} \frac{d v^{i}}{d t} d V+\iiint_{V} f^{i} v_{i} d V-\iint_{\Sigma} v_{i} \pi^{i k} d \sigma_{k} .
$$

Nous transformons la dernière intégrale par la formule de GrEen on intégrale de volume. Puis nous réunissons les trois derniers termes en une seule intégrale, soit:

$$
\iiint_{V}\left[-\mu v_{i} \frac{d v^{i}}{d t}+f^{i} v_{i}-\nabla_{k}\left(v_{i} \pi^{i k}\right)\right] d V
$$

En vertu des équations de mouvement du fluide [§ 15, éq. (15.5)]

$$
\mu \frac{d v^{i}}{d t}-f^{i}+\nabla_{k} \pi^{i k}=0
$$

nous avons :

$$
-\mu v_{i} \frac{d v^{i}}{d t}+f^{i} v_{i}-\nabla_{k}\left(v_{i} \pi^{i k}\right)=v_{i} \nabla_{k} \pi^{i k}-\nabla_{k}\left(v_{i} \pi^{i k}\right)=-\pi^{i k} \nabla_{k} v_{i}
$$

$\pi^{i k}$ est un tenseur symétrique, nous pouvons introduire la quantité

$$
\frac{1}{2} \pi^{i k}\left(\nabla_{i} v_{k}+\nabla_{k} v_{i}\right)=\pi^{i k} \nabla_{k} v_{i}
$$


La relation (19.1) se met alors sous la forme:

$$
\iiint_{V} \mu \frac{d \varepsilon}{d t} d V=\iint_{\Sigma} q^{i} d \sigma_{i}-\iiint_{V}^{1} \frac{1}{2} \pi^{i k}\left(\nabla_{i} v_{k}+\nabla_{k} v_{i}\right) d V .
$$

C'est l'expression intégrale du principe de conservation de l'energie pour un volume fluide $V$ en mouvement.

Nous transformons l'intégrale de surface en intégrale de volume et nous écrivons la relation obtenue sons la forme:

$$
\iiint_{V}\left[\mu \frac{d \varepsilon}{d \bar{t}}-\nabla_{i} q^{i}+\frac{1}{2} \pi^{i k}\left(\nabla_{i} v_{k}+\nabla_{k} v_{i}\right)\right] d V=0 .
$$

L'égalité précédente est vraie pour tout volume fluide du type considéré. En vertu des hypothèses de continuité. l'élément différentiel est identique. ment nul.

$$
\mu \frac{d \varepsilon}{d t}-\nabla_{i} q^{i}+\frac{1}{2} \pi^{i k}\left(\nabla_{i} v_{k}+\nabla_{k} v_{i}\right)=0
$$

Réciproquement si cette égalité a lieu en tout point du fluide, dans le domaine $D$, l'égalité intégrale (18.3) est vraie quel que soit le volume fluide $V$ considéré, limité par une surface fermée quelconque.

Nous pouvons énoncer le théorème.

ThÉoRime. - Si les diverses caractéristiques du fuide en mouvement sont définies comme des fonctions continues ainsi que leurs dérivées par rapport aux variables $x^{i}$, $t$, alors en tout point du domaine $D$ occupé par le fluide, on a l'égalité

$$
\mu \frac{d \varepsilon}{d t}=\nabla_{i} q^{i}-\frac{1}{2} \pi^{i k}\left(\nabla_{i} v_{k}+\nabla_{k} v_{i}\right)
$$

et pour tout volume fluide $V$, on a l'égalité

$$
\frac{d}{d t} \iiint_{V} \mu \varepsilon d V=\iint_{\Sigma} q^{i} d \sigma_{i}-\iiint_{V} \frac{1}{2} \pi^{i k}\left(\nabla_{i} v_{k}+\nabla_{k} v_{i}\right) d V .
$$

Dans le oas d'un fluide parfait, $\pi^{i k}=p g^{i k}$ :

$$
\frac{1}{2} \pi^{i k}\left(\nabla_{i} v_{k}+\nabla_{h} v_{i}\right)=p \nabla_{i} v^{i}
$$

En introduisant le volume spécifique $\omega=\frac{1}{\mu}$ et tenant compte de l'équation de continuité (15.4), on tire la formule remarquable

$$
\frac{d \varepsilon}{d t}=Q-p \frac{d \omega}{d t}
$$

où $Q=\omega \nabla_{i} q^{i}$. 
20. Forme différentielle du second principe.

L'entropie est une fonction additive de volume.

$$
S\left(V_{1}+V_{2}\right)=S\left(V_{1}\right)+S\left(V_{2}\right) \text {. }
$$

Pour un milieu continu, nous supposons qu'elle est definie par une densité d'entropie $\sigma$ en chaque point du milieu, $\sigma$ étant une fonction conti. núment différentiable. Chaque élément de masse $d m$ du milieu possède alors l'entropie :

$$
\sigma d m=\mu \sigma d V .
$$

L'entropie d'un volume fluide $V$ sera

$$
S=\iiint_{V} \mu \sigma d V
$$

$\sigma$ est une fonction des variables qui définissent l'état du milieu au point considéré. C'est done une fonction de $x^{i}, t$ soit directement, soit par l'intermédiaire de certaines caractéristiques du milieu.

DÉfinition. - Il entre dans l'expression $d u$ second principe le vecteur de composantes $\frac{q^{i}}{\theta}$, q $\mathfrak{x}^{\prime} i l$ est utile de distinguer dn vecteur courant de chaleur $q^{i}$. On sait en efiet que la notion de transformations réversibles et le second principe conduisent à une notion importante, celle des quantités de chaleur produites par des sources à des températures différentes.

Nous donnerons un nom au vecteur $\frac{q^{i}}{\theta}$ où $\theta$ est la température thermodynamique. Nous l'appellerons vecteur chaleur entropique et poserons:

$$
\vec{C}=\frac{\vec{q}}{\vec{\theta}} \text {. }
$$

Dans la suite, nous supposerons que les diverses caractéristiques du fluide considéré, sont des fonctions continues, admettant des dérivées continues, par rapport aux variables d'EULER $x^{i}$, $t$ ou aux variables de LAGRANGE $a^{i}, t$.

En portant la valeur (20.1) de $S$ dans (18.3), nous obtenons la relation suivante qui est vraie à chaque instant $t$ :

$$
\frac{d}{d t} \iiint_{V} \mu \sigma d V \geq \iint_{\Sigma} \frac{q^{i}}{\theta} d \sigma_{i} .
$$

Transformons l'intégrale de surface en intégrale de rolume et faisons usage du lemme (17.2). Nous obtenons l'inégalité

$$
\iiint\left[\mu \frac{d \sigma}{d t}-\nabla_{i}\left(\frac{q^{i}}{\theta}\right)\right] d V \geq 0 .
$$


L'inégalité précédente a lieu à tout instant et pour tout volume fluide $V$. En vertu des hypothèses de continuité, nous en déduisons que l'élément différentiel satisfait nécessairement à l'inégalité de même sens:

$$
\mu \frac{d \sigma}{d t}-\nabla_{i}\left(\frac{q^{t}}{\theta}\right) \geq 0 \text {. }
$$

Cette inégalité est vraie en tout point du domaine $D$ et à tout instant, il en résulte que-pour tont volume fluide $V$ nous avons l'inégalité intégrale de même sens (20.4).

Nous énonçons le théorème.

THн́ов亡̀me. - Si les diverses caractéristiques d'un fluide en mouvement sont définies comme des fonctions continues ainsi que leurs dérivées par rapport aux variables $x^{i}, t$, alors dans les hypothèses du lemme (17.2), en tout point $d u$ domaine $D$ occupé par ce fuide, on a l'inégalité :

$$
\mu \frac{d \sigma}{d t} \geq \nabla_{i}\left(\frac{q^{t}}{\theta}\right)
$$

et pour tout volume fluide $V$ limité par une surface régulière fermée $\Sigma$, on a l'inégalité

$$
\frac{d}{d t} \iiint_{V} \mu \sigma d V \geq \iint_{\Sigma} \frac{q^{i}}{\theta} d \sigma_{i} .
$$

\section{II. - L'extension relativiste des principes de la thermodynamique.}

21. I'interprétation des équations du fluide thermodynamique relativiste.

Pour avoir une interprétation en termes de temps et d'espace, des équations relativistes du fluide thermodynamique (c.. $\S 13$ ), nous allons prendre pour $V_{4}$, l'espace-temps de la relativité restreinte. Nous le rapporterons à un système de coordonnées galiléennes réduites $\left(x^{0}=c t, x^{i}\right)$.

Considérons le fluide au voisinage d' un point $P$.

Introduisons un système d'axes galiléens orthogonaux $T$ d'origine $P$. Par rapport à $T$, le point $P$ du fluide est au repos. Nous avons, en fonction du vecteur d'espace $v^{i}=\frac{d x^{i}}{d t}$, les formules

$$
u^{0}=\frac{1}{\sqrt{1-\beta^{2}}} \quad u^{i}=\frac{v^{i}}{c \sqrt{1-\beta^{2}}} \quad\left(\beta=\frac{v}{c}\right) .
$$

Il en résulte qu'au point $P, u^{0}=1, u^{i}=0$. Mais les dérivées de $u^{i}$ ne sont pas nulles

$$
\partial_{\lambda} u^{0}=0 \quad \partial_{\lambda} u^{i}=\frac{1}{c} \frac{\partial_{\lambda} v^{i}}{\sqrt{1-\beta^{2}}} .
$$


Nous nous proposons de mettre les équations du fluide thermodynamique sous une forme analogue à la suivante:

$$
\begin{aligned}
& \frac{\partial \rho}{\partial t}+\partial_{k} p^{k}=\Phi^{0} \\
& \frac{\partial p^{i}}{\partial t}+\partial_{k} \pi^{i k}=\Phi^{i}
\end{aligned}
$$

où $p^{i}$ est le vecteur impulsion relativiste auquel toutes les formes d'energie doivent apporter leur contribution. $\pi^{i k}$ est le tenseur des pressions des forces superficielles, $\Phi^{i}$ les composantes de la résultante des forces de masse, $\Phi^{u}$ la quatrième composante de la force d'univers, doit représenter un travail $\left({ }^{3}\right)$.

Ces préliminaires étant posés, il est plus simple de partir des conditions de conservation $\nabla_{a} T^{\alpha \beta}=0$ imposées au tenseur d'impulsion-énergie

$$
T^{\alpha \beta}=\rho u^{\alpha} u^{\beta}-\pi^{\alpha \beta}-\left(u^{\alpha} q^{\beta}+u^{\beta} q^{\alpha}\right)
$$

à savoir les quatre équations :

$$
\begin{aligned}
& \partial_{\alpha}\left(\rho u^{\alpha} u^{0}\right)-\partial_{\alpha} \pi^{\alpha}-\partial_{\alpha}\left(u^{\alpha} q^{0}+u^{0} q^{\alpha}\right)=0 \\
& \partial_{\alpha}\left(\rho u^{\alpha} u^{i}\right)-\partial_{\alpha} \pi^{\alpha i}-\partial_{\alpha}\left(u^{\alpha} q^{i}+u^{i} q^{\alpha}\right)=0
\end{aligned}
$$

Nous interprétons ces équations en tenant compte des conditions

$$
u_{\alpha} \pi^{\alpha \beta}=0 \quad u_{x} q^{\alpha}=0 .
$$

Multiplions (21.4) par $u_{0}$

$$
\partial_{\alpha}\left(\rho u^{\alpha}\right)-\partial_{\alpha}\left(u_{0} \pi^{0 \alpha}\right)-u_{0} \partial_{x}\left(u^{\alpha} q^{0}+u^{0} q^{\alpha}\right)=0 .
$$

Or $u_{0} \pi^{\theta \alpha}=-u_{i} \pi^{\alpha i}$, nous en déduisons :

$$
\partial_{0}\left(\rho u^{0}\right)-\partial_{k}\left(\rho u^{k}-q^{k}+u_{i} \pi^{i k}\right)=\dot{\grave{z}}_{0} q^{0}+u^{0} \partial_{0}\left(u_{0} q^{0}\right) \text {. }
$$

Une transformation analogue appliquee ì (21.5) donne si nous nous limitons aux infiniment petits d'ordre 1 en $\beta$

$$
\partial_{0}\left(\rho u^{i}-q^{i}+u_{k} \pi^{i k}\right)-\partial_{k} \pi^{i k}=\partial_{k}\left(u^{k} q^{i}+u^{i} q^{k}\right)
$$

Ainsi, au point $P_{0}$, les équations (21.4) et (21.5) peuvent s'écrire, aux infiniment petits d'ordre supérieur à 1 en $\beta$ près:

$$
\begin{gathered}
\partial_{0}\left(\rho u^{0}\right)+\partial_{k}\left(\rho u^{k}-q^{k}+\pi^{i k} v_{i}\right)=\partial_{0} q^{\prime \prime}+u^{0} \partial_{0}\left(u_{0} q^{0}\right) \\
\partial_{0}\left(\rho u^{i}-q^{i}+u_{k} \pi^{i k}\right)-\partial_{k} \pi^{i k}=\partial_{k}\left(u^{i} q^{k}+u^{k} q^{i}\right) .
\end{gathered}
$$

On voit qu'il est possible d'interpréter le vecteur

$$
\left(\rho u^{i}-q^{i}+u_{k} \pi^{i k}\right)
$$

(9) Le lecteur peut trouver un exposé clair de la Thérie de la Relativité restreinte dans les Eléments de Oalcul tensoriel de Lichinerowicz [17]. 
comme ur vecteur impulsion $p^{i}$. Il est plus délicat d'interpréter les seconds membres des équations précédentes. Mais du point de vue théorique, en $P_{0}$ nous avons

$$
a_{k}\left(u^{i} q^{k}+u^{k} q^{i}\right)=q^{i} \partial_{k} u^{k}+q^{k} \partial_{k} u^{i}
$$

Cette quantité prend les dimensions $\mathrm{d}^{\prime}$ une force, si on considère $q^{i}$ comme une impulsion. La quantité précédente représente, si j'ose dire, une force d'origine thermodynamique.

Quant au seeond membre de $\left(21.4^{\prime}\right)$, on a d'abord

$$
\begin{gathered}
\partial_{0} q^{0}=\partial_{0} q_{0}=-x \partial_{0}\left[\partial_{0} \theta-\left(u^{\lambda} \partial_{\lambda} \theta\right) u_{0}\right]=x \partial_{i} \theta u^{0} \partial_{0} u^{i} \\
\partial_{0} q^{0}=-q_{i} u^{0} \partial_{0} u^{i} .
\end{gathered}
$$

Puis de $u_{x} q^{\alpha}=0$, on déduit que

$$
u^{0} \partial_{0}\left(u_{0} q^{0}\right)=-u^{0} \partial_{0}\left(u_{i} q^{i}\right)=-q^{i} u^{0} \partial_{0} u^{i}
$$

$\partial_{0} q^{0}$ et $u^{0} \partial_{0}\left(u_{0} q^{c}\right)$ ont les dimensions d' un travail. Il représente, dans le mème ordre d'idées, un travail d'origine thermodynamique.

Si nons utilisons la variable temps ordinaire $t$, il faut l'identifier au facteur $c$ près, avec l'arc de ligne de courant en $P_{0}$. Les équations (21.4') et $\left(21.5^{\prime}\right)$ s'écriront alors avec des grandeurs exprimées en unités $C G S$ :

$$
\begin{aligned}
& \frac{\partial}{\partial t} \frac{\rho}{\sqrt{1-\beta^{2}}}+\partial_{k}\left(\frac{\rho v^{k}-\frac{1}{c^{2}} q^{k} \sqrt{1-\beta^{2}}+\frac{1}{c^{2}} v_{i} \pi^{i k}}{\sqrt{1-\beta^{2}}}\right)=-\frac{2}{c^{2}} q^{i} \frac{\partial}{\partial t} \frac{v_{i}}{\sqrt{1-\beta^{2}}} \\
& \frac{\partial}{\partial t}\left(\frac{\rho v^{i}-\frac{1}{c^{2}} q^{i} \sqrt{1-\beta^{2}}+\frac{1}{c^{2}} v_{k} \pi^{i k}}{\sqrt{1-\beta^{2}}}\right)-\frac{1}{c^{2}} \partial_{k} \pi^{i k}=\frac{1}{c^{2}} \partial_{k}\left(\frac{v^{i} q^{k}+v^{i} q^{k}}{\sqrt{1-\beta^{2}}}\right)
\end{aligned}
$$

où $v_{i}$ sont les composantes covariantes du vecteur vitesse d'espace $\vec{v}$ dans la métrique $-\left(d x^{1}\right)^{2}-\left(d x^{2}\right)^{2}-\left(d x^{3}\right)^{2}$.

Les équations (21.6) et (21.7) apportent des corrections relativistes aux équations classiques de la dynamique des milieux continus. Ces corrections sont de l'ordrę de $\frac{1}{c^{2}}$. On conçoit la difficulté qu'éprouve l'expérience à s'en rendre compte.

22. L'équation de continuité relativiste et les principes de conservation classique.

Nous allons revenir un instant aux équations classiques du fluide, sous la forme qui a été introduite au cours de la section précédente. Cependant, du point de vue théorique, nous pouvons supposer que la force $f^{i}$ dérive d'un tenseur symétrique, soit $f^{i}=\nabla_{k} \omega^{i k}$. Nous faisons donc entrer $f^{i}$ dans 
le tenseur général des pressions. Et nous considérons les équations classiques sous la forme:

$$
\begin{gathered}
\mu \frac{d v^{i}}{d \bar{t}}+\nabla_{k} \pi^{i k}=0 \\
\frac{d \mu}{d t}+\nabla_{i} v^{i}=0 \\
\mu \frac{d \varepsilon}{d t}-\nabla_{i} q^{i}+\pi^{i k} \nabla_{i} v_{k}=0 .
\end{gathered}
$$

Multiplions (22.1) par $\frac{1}{c^{2}} v_{i}$ en sommant par rapport à l'indice $i$; (22.2) $\operatorname{par} \frac{1}{c^{2}}\left(\frac{1}{2} v^{2}+\varepsilon\right)$ et $(22.3)$ par $\frac{1}{c^{2}}$ : nous avons :

$$
\begin{gathered}
\frac{1}{c^{2}} \mu \frac{d}{d t}\left(\frac{1}{2} v^{2}\right)+\frac{1}{c^{2}} v_{i} \nabla_{k} \pi^{i k}=0 \\
\frac{1}{c^{2}}\left(\frac{1}{2} v^{2}+\varepsilon\right) \frac{d \mu}{d t}+\frac{1}{c^{2}} \mu\left(\frac{1}{2} v^{2}+\varepsilon\right) \nabla_{i} v^{i}=0 \\
\frac{1}{c^{2}} \mu \frac{d \varepsilon}{d t}-\frac{1}{c^{2}} \nabla_{i} q^{i}+\frac{1}{c^{2}} \pi^{i k} \nabla_{i} v_{k}=0 .
\end{gathered}
$$

En ajoutant membre à membre les équations (22.2) (22.4) (22.5) et (22.6), nous obtenons l'égalité

$$
\frac{d}{d t}\left[\mu\left(1+\frac{1}{2} \frac{v^{2}}{c^{2}}+\frac{\varepsilon}{c^{2}}\right)\right]+\mu\left(1+\frac{1}{2} \frac{v^{2}}{c^{2}}+\frac{\varepsilon}{c^{2}}\right) \nabla_{i} v^{i}-\frac{1}{c^{2}} \nabla_{k} q^{k}+\frac{1}{c^{2}} \nabla_{k}\left(v_{i} \pi^{i k}\right)=0
$$

que nous écrivons sous la forme

$$
\frac{\partial}{\partial t}\left[\mu\left(1+\frac{1}{2} \frac{v^{2}}{c^{2}}+\frac{\varepsilon}{c^{2}}\right)\right]+\nabla_{k}\left[\mu\left(1+\frac{1}{2} \frac{v^{2}}{c^{2}}+\frac{\varepsilon}{e^{2}}\right) v^{k}-\frac{1}{e^{2}} q^{k}+\frac{1}{c^{2}} v_{i} \pi^{i k}\right]=0
$$

En comparant cette equation à l'équation (21.6) du paragraphe précédent, on voit que l'équation (22.7) est une approximation classique de l'équation de continuité relativiste, puisque dans cette dernière $\rho$ doit représenter la densité de matière et d'énergie réunie et que l'énergie cinétique provient du facteur $\frac{1}{\sqrt{1-\beta^{2}}}$ de $\rho$.

Du point de vue classique, on a formulé les deux principes de conservation de la matière et de l'énergie. La conservation de l'énergie se traduit par la relation

$$
d U=Q_{e}-\widetilde{\sigma}_{e}
$$


Et à côté de la distinction entre matière et énergie, on a introduit une seconde distinction importante entre l'énergie d'origine mécanique $\mathfrak{G}_{e}$ et l'énergie d'origine calorifique $Q_{e}$, suivant leurs modes de production.

La théorie de la relativité n' impose plus la distinction nécessaire entre la matière et l'énergie. Et, à partir des considérations précédentes, nous voyons que les lois de conservation classiques se trouvent implicitement contenues dans les équations d' EINSTEIN

$$
S_{\alpha \beta}=\chi T_{\alpha \beta}
$$

Ces lois s'expriment à travers les identités de conservation imposées an tenseur d'impulsion-énergie $T^{\alpha \beta}$. Nous sommes nécessairement conduits à prendre l'équation

$$
u_{\beta} \nabla_{\alpha} T^{\alpha \beta}=0
$$

comme l'équivalent relativiste des principes de conservation.

Cette equation n'est autre que l'équation de continuité relativiste. Elle appelle denx remarques :

1. - la distinction entre chaleur et travail, comme modes de production d'énergie, est conservée en relativité. Cette distinction se traduit par la nature géométrique différente $d u$ tenseur thermodynamique $Q^{\alpha \beta}=\left(u^{\alpha} q^{\beta}+u^{\beta} q^{x}\right)$, et du tenseur de la matière pure $\rho u^{\alpha} u^{\beta}$.

2. - le choix précédent satisfait aux exigences d'interprêtation locale de la physique. Il nous suffit de poser :

$$
\rho=\mu(1+\varepsilon)
$$

et d'interpréter $\mu$ comme la densité de matière locale. Celle-ci doit vérifier l'équation:

$$
\nabla_{\alpha}\left(\mu u^{\alpha}\right)=0
$$

23. La forme entropie.

Nous venons de faire remarquer que la distinction entre l'énergie-chaleur et l'énergie-travail est conservée en relativité. Nous concevons donc l'utilité de la formulation d'une thermodynamique relativiste. Or les lois de conservation ne sont qu' un corollaire des principes de la théorie de la relativité. Par contre lá notion d'entropie nous oblige à une réflexion nouvelle. Elle doit jouer un rôle fondamental, en ce sens qu'elle déterminera tout le caractère de la thermodynamique relativiste.

Avant de procéder à la formulation de l'extension relativiste du second principe, nous allons introduire une définition invariante de l'entropie.

Soit un domaine $D_{4}$ de l'espace-temps, occupé par un milieu thermo. dynamique. Nous considérons des éléments de fluide et nous suivons leur variation dans l'espace-temps comme au $\$ 9$. Nous rapportons d'abord chaque élément de fluide au repère propre associé. Puis, par un changement de 
coordonnées, nous en déduisons l'expression invariante générale des formules étudiées.

Choisissons dans $D_{4}$ des coordonnées telles que les lignes où la variable $x^{\prime \prime}$ varie seule, soient les lignes de courant. Et considérons une hypersurface definie localement par l'équation $x^{0 \prime}=$ const., soit

$$
x^{0 \prime}=0
$$

qui définit dans $D_{4}$ un domaine à trois dimensions $D_{3}$.

Chaque point de $D_{3}$ sera caractérisé par ses coordonnés $x^{\prime \prime}$. L'élément de fluide au point $\left(x^{\prime \prime}\right) \in D_{3}$, est caractérisé par sa masse $m$, son volume ordinaire $\omega^{\prime \prime} \wedge \omega^{2 \prime} \wedge \omega^{3 \prime}$. Si $\rho$ est la densité propre du fluide au point consi. déré, cet élément de fluide possède une entropie propre

$$
\sigma m=\rho \sigma\left(\omega^{1 \prime} \wedge \omega^{2 \prime} \wedge \omega^{3 \prime}\right.
$$

ou $\sigma$ est la densité propre d'entropie. C'est une fonction scalaire des caracté. ristiques da fluide.

Nous avons vu au $\S 20$, comment on considère la variation de l'entropie dans le temps. Cette variation est rapportée au temps propre. Or, en relativité, le temps propre subit une variation relative à la coordonnée $x^{0^{\prime}}$ de sorte qu' en réalite nous considérons dans le repère propre la variation de la quantité

$$
\rho \circ u^{0 \prime} \text {. }
$$

Il se trouve ainsi introduit le vecteur qui a pour composante non nulle pou $u^{\prime \prime}$ dans le repère propre. Nous sommes amenés à considérer le champ de vecteurs po $\vec{u}$ ainsi défini dans $D_{4}$. Dans un repère naturel associé à un système de coordonnées locales quelconque, ce champ de vecteurs est défini par ses composantes contravariantes

$$
\text { poú. }
$$

Le vecteur $\rho \sigma \vec{u}$ admet le tensenr polaire d'ordre 3 dont les composantes sont données par:

$$
(\rho \sigma)_{\alpha_{1} \alpha_{2} x_{3}}^{*}=\eta_{\alpha_{0} x_{1} \alpha_{2} \alpha_{3} \rho} \rho \sigma u^{x_{0}}
$$

où $\eta_{x_{0} \alpha_{1} \alpha_{2} \alpha_{3}}$ est le tenseur complètement antisymétrique associé à la forme élément de volume. Si $\varepsilon_{x_{0} \alpha_{1} x_{2} x_{3}}$ désigne l'indicateur de KRONECKER, on a

$$
\eta_{\alpha_{0} \alpha_{1} x_{2} \alpha_{3}}=\sqrt{|g|} \varepsilon_{\alpha_{0} x_{1} \alpha_{2} \alpha_{3}} \text {. }
$$

Nous pouvons faire correspondre au champ de vecteurs $\rho \sigma \vec{u}$ d'une manière invariante, la forme différentielle extérieure d'ordré 3 :

$$
\boldsymbol{Q}=\underset{\alpha_{1}<\alpha_{3}<\alpha_{8}}{\Sigma}(\rho \sigma)_{\alpha_{1} \alpha_{3} \alpha_{3}}^{*} d x^{\alpha_{1}} \wedge d x^{\alpha_{2}} \wedge d x^{\alpha_{s}}=\frac{1}{3 !} \eta_{\alpha_{0} \alpha_{3} \alpha_{2} \alpha_{3} \rho} \rho \sigma u^{\alpha_{0}} d x^{\alpha_{1}} \wedge d x^{\alpha_{2}} \wedge d x^{\alpha_{s}}
$$


Phan MaU QuaN: Sur we thépie relativiste ales fluides, etc.

Nous introduisons les définitions suivantes.

Dh́finitrons. - La forme différentielle $Q$ d'ordre 3 définie par la formule (23.3) sera appelée forme entropie attachée à l'élément de fluide au point $\left(x^{a}\right)$.

$$
\mathbf{Q}=\frac{1}{3 !} \eta_{x_{0} \alpha_{1} x_{2} x_{8}} \rho \sigma u^{\alpha_{0}} d x^{\alpha_{1}} \wedge d x^{\alpha_{2}} \wedge d x^{\alpha_{3}}
$$

Soit un domaine à trois dimensions $B_{3}$ pris dans le domaine $D_{3}$ comme il a été défini plus haut.

On appellera entropie du milieu considéré, dans le domaine à trois dimensions $B_{3}$, l'intégrale de la forme entropie $Q$ sur ce domaine.

$$
\iiint_{B_{s}} \boldsymbol{Q}=\iiint_{B_{s}} \frac{1}{3 !} \eta_{x_{0} \alpha_{1} \alpha_{2} \alpha_{3} \rho \sigma u^{\alpha_{0}}} d x^{\alpha_{1}} \wedge d x^{\alpha_{2}} \wedge d x^{\alpha_{3}}
$$

24. L'extension relativiste du second principe de la thermodynamique.

Dans la suite des raisonnements, nous supposerons que les diverses quantités introduites sont des fonctions continues et à dérivées continues dans le domaine considéré de l'espace-temps.

Soit donc le domaine $D_{4}$ de l'espace-temps, occupé par un milieu thermodynamique. Nous considérons dans ce domaine, un tube de courant $T_{3}$ et des sections $B_{3}$ de ce tube, du type qui a été défini dans le paragraphe précédent. Nous obtenons ainsi un domaine spatio-temporel $B_{4}$.

Chaque section $B_{3}$ définit un domaine à trois dimensions auquel est attachée l'entropie

$$
S=\iiint_{B_{3}} \Omega=\iiint_{B_{3}} \frac{1}{3 !} \eta_{x_{0} \alpha_{1} \alpha_{2} \alpha_{3} \rho \sigma u^{\circ} 0} d x^{\alpha_{1}} \wedge d x^{\alpha_{2}} \wedge d x^{\alpha_{3}} .
$$

Lorsque la section $B_{3}$ varie dans le tube de courant $T_{3}$, son entropie varie également. Nous appellerons variation de l'entropie $S$, quand la section $B_{3}$ se déplace d'une position $B_{3}^{(1)}$ à une position $B_{3}^{(2)}$, la différence:

$$
S_{2}-S_{1}=\iiint_{B_{3}^{(2)}} \Omega-\iiint_{B_{3}^{(1)}} \mathbf{\Omega} .
$$

Nous pouvons expliciter cette formule.

On a en effet

$$
\iiint_{T_{3}} \Omega=0
$$


et par suite

$$
S_{2}-S_{1}=\iiint_{\partial B_{4}} \Omega
$$

où $\partial B_{4}$ est le bord de $B_{4}$. En appliquant la formule de STokms il vient

$$
S_{2}-S_{1}=\iiint \int_{B_{4}} D Q
$$

où $D \Omega$ est la différentielle extérieure de $\Omega$ et où l'intégration est étendue au domaine à quatre dimensions $B_{4}$ limité par le tube de courant $T_{3}$ et par les deux sections $B_{3}^{(1)} B_{3}^{(2)}$.

La formule (24.2) est invariante pour tout changement de coordonnées. En nous reportant à la définition de la forme $\Omega$, nous avons:

$$
D \mathbf{Q}=\sum_{\alpha=0}^{3} \frac{\partial\left(\rho \sigma u^{\alpha} \sqrt{|g|}\right)}{\partial x^{\alpha}} d x^{0} \wedge d x^{1} \wedge d x^{2} \wedge d x^{3} .
$$

La quantité $\sum_{\alpha=0}^{3} \frac{\partial\left(\rho \sigma u^{\alpha} \sqrt{|g|}\right)}{\partial x^{\alpha}}$ définit un tenseur complètement antisymétrique d'ordre 4. En introduisant son scalaire polaire

$$
\frac{1}{V \bar{g} \mid} \sum_{\alpha=0}^{3} \frac{\partial\left(\rho \sigma u^{\alpha} \sqrt{|g|}\right)}{\partial x^{\alpha}}=\nabla_{\alpha}\left(f \sigma u^{\alpha}\right)
$$

nous obtenons

$$
S_{2}-S_{1}=\iiint \int_{B_{4}} \nabla_{x}\left(\rho \sigma u^{\alpha}\right) \sqrt{|g|} d x^{0} \wedge d x^{4} \wedge d x^{2} \wedge d x^{3} .
$$

L'intégrale du second membre est étendue au domaine $B_{4}$.

Par definition, nous dirons que sa valeur est la variation d'entropie attachée au domaine $B_{4}$.

Nous avons ainsi defini l'entropie du milieu dans un domaine à trois dimensions $B_{3}$, la variation d'entropie attachée à un domaine à quatre dimensions $B_{4}$.

Nous introduisons le vecteur $\vec{C}$ qui a pour composantes dans le repère propre,

$$
C^{0 \prime}=0 \quad C^{\prime \prime}=\frac{q^{\prime \prime}}{\theta} .
$$

Dans un repère naturel quelconque, $\vec{C}$ aura pour composantes

$$
C^{\alpha}=\frac{q^{\alpha}}{\theta}
$$

où $q^{x}$ désigne le vecteur courant de chaleur. 
Pham MaU Quan: Sur une théorie relativiste des fluides, etc.

Le vecteur $\vec{C}$ ainsi défini sera encore appelé vecteur chaleur entropique.

Pour faire l'extension relativiste du second principe de la thermodyna. mique, nous allons considérer des domaines à quatre dimensions $B_{4}$, limités par des tubes de courant $T$ et par des sections $B_{3}$ que nous supposons maintenant engendrées par des lignes de chaleur. Nous postulons que la variation d'entropie attachee à tout domaine $B_{4}$ ainsi construit, est líe au flux du vecteur chaleur entropique $\vec{C}$ à travers le tube latéral $T$ par l'inégalité

$$
S_{2}-S_{1} \geq \iiint_{T} \frac{q^{\alpha}}{\theta} d \omega_{\alpha}
$$

où $d \omega_{\alpha}$ désignera l'élément d'aire de la frontière $\partial B_{4}$ de $B_{4}$.

Or

$$
S_{2}-S_{1}=\iiint \int_{B_{4}} D \Omega
$$

et

$$
\iiint_{T} \frac{q^{\alpha}}{\theta} d \omega_{\alpha}=\iiint_{\partial B_{4}} \frac{q^{\alpha}}{\theta} d \omega_{x}
$$

Des égalitês (24.3), nous déduisons après avoir transformé l'intégrale de flux en intégrale de divergence :

$$
\iiint \int_{B_{4}}\left[\nabla_{\alpha}\left(\rho \sigma u^{x}\right)-\nabla_{\alpha}\left(\frac{q^{\alpha}}{\theta}\right)\right] \sqrt{|g|} d x^{0} \wedge d x^{1} \wedge d x^{2} \wedge d x^{3} \geq 0 .
$$

Cette inégalité a lieu pour tout domaine $B_{4}$ du type défini précédemment. Nous allons montrer qu' en vertu des hypothèses de continuité, elle entraîne $q u$ ' en tout point du domaine $D_{4}$ :

$$
\nabla_{\alpha}\left(\rho \sigma u^{\alpha}\right)-\nabla_{\alpha}\left(\frac{q^{\alpha}}{\theta}\right) \geq 0
$$

En effet supposons que l'inégalité contraire a lieu en un point $P \in D_{4}$. Par raison de continuité, il existerait un voisinage $V(P)$ de ce point, pour lequel on aurait

$$
\iiint \int_{V(\mathcal{P})}\left[\nabla_{a}\left(\rho \sigma \boldsymbol{u}^{\alpha}\right)-\nabla_{a}\left(\frac{q^{\alpha}}{\theta}\right)\right] \sqrt{|g|} d x^{0} \wedge d x^{1} \wedge d x^{2} \wedge d x^{3}<0 .
$$

Or, dans ce voisinage, il est toujours possible de construire un tube de courant limité par deux sections engendrées par des lignes de chalear et 
contenant à son intérieur le point $P$. Ce domaine $B_{4}^{\prime}$ est du type $B_{4}$ : on a donc

$$
\iiint \int_{B_{i^{\prime}}}\left[\nabla_{\alpha}\left(\rho \sigma u^{\alpha}\right)-\nabla_{\alpha}\left(\frac{q^{\alpha}}{\theta}\right)\right] \sqrt{|g|} d x^{0} \wedge d x^{1} \wedge d x^{2} \wedge d x^{3} \geq 0
$$

comme conséquence du postulat (24.4'). L'inégalité (24.6) est donc impossible, sinon elle entraine contradiction.

L'inégalite (24.5) est donc vraie en tout point du domaine $D_{4}$. On en tire:

$$
\nabla_{\alpha}\left(\rho \sigma u^{x}\right) \geq \nabla_{\alpha}\left(\frac{q^{\alpha}}{\theta}\right)
$$

et par intégration sur un domaine $B$ à quatre dimensions, limité par une frontière régulière quelconque (et non plus nécessairement $d u$ type construit dans le postulat), on en deduit:

$$
\iiint \int_{B} D \Omega \geq \iiint_{\partial B} \frac{q^{x}}{\theta} d \omega_{\alpha} .
$$


CHAPITRE IV.

\section{LA STRUCTURE DES EQUATIONS DU CHAMP RELATIF}

A UN FLUIDE PARFAIT THERMODYNAMIQUE

\section{I. - Le problème de Cauchy.}

\section{Position du problème.}

Etant donné dans la variété espace-temps $V_{4}$ de la relativité générale, un domaine $D$ occupé par un fluide parfait thermodynamique, on se propose d'étudier la structure des équations fondamentales du champ, au moyen de la solution du problème de CaUCHY.

Nous nous límitons au cas où la constante cosmologique est nulle (cf. \& 5). Les modifications à faire subir à l'étude, lorsqu'il existe une constante cosmologique, sont aisées à obtenir.

A l'extérieur du domaine $D$, le champ de gravitation satisfait aux équations d' Einstein sans second membre

$$
S_{\alpha \beta} \equiv R_{\alpha \beta}-\frac{1}{2} R g_{\alpha \beta}=0 .
$$

A l'intérieur du domaine $D$, il est supposé satisfaire aux équations d'Einstein

$$
S_{\alpha \beta} \equiv R_{\alpha \beta}=\frac{1}{2} R g_{x \beta}=\chi T_{\alpha \beta}
$$

où le tenseur d'impulsion-énergie $T_{\alpha \beta}$ correspond au fluide parfait thermodynamique :

$$
T_{\alpha \beta}=(\rho+p) u_{\alpha} u_{\beta}-p g_{\alpha \beta}-\left(u_{\alpha} q_{\beta}+u_{\beta} q_{\alpha}\right)
$$

$q_{x}$ est le vecteur courant de chaleur, lié au champ de température par les relations :

$$
\begin{gathered}
q_{x}=-x \partial_{\rho} \theta\left(g_{\alpha}^{\rho}-u \rho u_{\alpha}\right) \\
\nabla_{\alpha} q^{\alpha}=c \rho u^{\alpha} \partial_{\alpha} \theta-\frac{l}{\rho} u^{\alpha} \partial_{\alpha} \rho .
\end{gathered}
$$

Nous supposerons que l'équation d'etat $d u$ fluide parfait est prise sous la forme

$$
\rho=\varphi(p, \theta)
$$

et nous poserons dans la suite

$$
\partial^{\lambda} \theta=g^{\lambda \rho} \partial_{\rho} \theta \quad Z=u^{\alpha} \partial_{\alpha} \theta .
$$


Les équations (25.2) peuvent s' écrire

$$
R_{\alpha \beta}=S_{\alpha \beta}-\frac{1}{2} S g_{\alpha \beta}
$$

où $S$ désigne l'invariant $g^{\alpha \beta} S_{\alpha \beta}$ qui a pour valeur:

$$
S=\chi(p-3 p)
$$

Si on remarque que $R=-S$, on conclut que la courbure scalaire de l'epace-temps n'est pas affectée par le champ de température.

Notre etude purement locale sera d'abord relative aux equations d' EissteIN sans second membre. Ce problème a reçu une solution effective par G. Darmors et A. Lichnerowicz et dans un autre ordre d'idées par Mme Foukés qui suppose seulement les hypothèses de différentiabilité. Nous nous contentons donc d'en rappeler les résultats fondamentaux, qui nous seront utiles pour simplifier l'exposé de la solution des équations avec second membre (25.2).

26. La solution des équations sans second membre.

Les équations sans second membre (25.1) correspondent d'ailleurs an domaine de l'espace-temps non balayé par l'énergie. Ces équations s'écrivent indifféremment d'après (25.3), soit $S_{\alpha \beta}=0$, soit

$$
R_{\alpha \beta}=0 \text {. }
$$

Le champ de gravitation étudié se trouve déterminé, par définition, par ses potentiels $g_{\alpha \beta}$ et leurs dérivées premières. Le problème est done le suivant:

Le problème extérieur. - Etant donnés, sur une hypersurface $S$, les potentiels de gravitation et leurs dérivées premières, déterminer en dehors de $S$, les valeurs des potentiels supposés satisfaire aux équations (26.1).

Nous supposons que l'hypersurface $S$ est partout orientee dans l'espace et qu'elle est localement représentée par l'équation

$$
x^{0}=0 .
$$

Nous appellerons indice d'une dérivée de $g_{\alpha \beta}$, le nombre de fois que l'indice 0 figure sous le symbole de dérivation.

La donnée sur $S$ deś potentiels $g_{\alpha \beta}$, détermine les valeurs sur $S$ de leurs dérivées tangentes $\partial_{i} g_{a \beta}$. Les données de CAUCHY sont alors constitúes par les valeurs sur $S$ des potentiels $g_{x \beta}$ et de leurs dérivées premières d'indice 1 , soit $\partial_{0} g_{\alpha \beta}$. Et nous nous proposons de déterminer les valeurs sur $S$, des dérivées d'indice supérieur à 1 . 
Phan MaU Quan: Sur une thérie relativiste des fluides, etc.

Les seules dérivées qui puissent être discontinues à la traversée de $S$, sont les dérivées d'indice $2: \partial_{00} g_{\alpha \beta}$. Nous les mettons done en évidence dans nos équations (26.1) qui s'écrivent plus explicitement:

$$
\left\{\begin{array}{l}
R_{i j} \equiv-\frac{1}{2} g^{00 \partial_{00} g_{i j}+F_{i j}(d \cdot c)=0} \\
R_{i 0} \equiv \frac{1}{2} g^{0 j} \partial_{00} g_{i j}+\Phi_{i}(d \cdot c)=0 \\
R_{00} \equiv-\frac{1}{2} g^{i j} \partial_{00} g_{i j}+\psi(d \cdot c)=0
\end{array}\right.
$$

où les $F_{i j}(d \cdot c), \Phi_{i}(d \cdot c)$ et $\psi(d \cdot c)$ désignent des quantités calculables sur $S$, à partir des données de CAUCHY $(d \cdot c)$ par opération algébrique on par dérivation sur $S$.

Il est à noter qu'aucune des équations ne contient les dérivées $\partial_{0 n} g_{0 \lambda}$. Ceci s'explique par le fait que la donnée sur $S$ des potentiels et de leurs dérivées premières, laisse subsister la possibilité de changements de variables conservant $S$ point par point et les valeurs sur $S$ des données de CaUCHY. De tels changements de variables ne modifient pas les valeurs des $\partial_{00} g_{i j}$, mais permettent de donner à $\partial_{00} g_{\lambda 0}$ des valeurs arbitraires. Les discontinuités de $\partial_{00} g_{\lambda_{0}}$ apparaissent comme des singularités artificielles et sont dépourvues de signification physique.

A cette réserve près, les dérivées secondes des potentiels sont continues pour toute hypersurface $S$ telle que $g^{00} \neq 0$. Il est clair que les memes conclusions s'étendent aux dérivées successives des potentiels, dans le cas où les données de $\mathrm{C}_{A U C H Y}$ sont dérivables à un ordre supérieur.

Cette étude ne fait intervenir que les équations (26.3).

Ceci étant dit, il est facile d'établir les identités:

$$
\begin{gathered}
g^{0 j} R_{i j}+g^{00} R_{i 0}=R_{i}^{0}=S_{i}^{0} \\
\frac{1}{2}\left(g^{00} R_{00}-g^{i j} R_{i j}\right) \equiv S_{0}^{0},
\end{gathered}
$$

Le système dęs équations (26.3) et (26.4) équivaut alors au système

$$
\begin{aligned}
& R_{i j}=0 \\
& S_{\alpha}^{0}=0 .
\end{aligned}
$$

La forme des équations (26.3) et 26.4) montre que ${ }_{2}^{*}$ les premiers membres des relations (26.5), donc les $S_{\alpha}^{0}$, ne contiennent aucune dérivée d'indice 2 des potentiels. Les équations (26.7) fournissent alors quatre équations que doivent nécessairement vérifier les données de CaUCHY. 
Les $S_{x}^{0}$ sont liées aux premiers membres des équations (26.6) par les conditions de conservation $\nabla_{x} S_{\beta}^{\alpha}=0$, soit

$$
\nabla_{0} S_{x}^{0}+\nabla_{k} S_{x}^{k}=0
$$

Or, pour une solution des équations (26.6), on a:

$$
\begin{gathered}
S_{j}^{i}=g^{i 0} R_{0 j .}-\frac{1}{2} \delta_{j}^{i}\left(g^{00} R_{00}+2 g^{0 k} R_{0 k}\right) \\
S_{0}^{i}=g^{i \lambda} R_{\lambda 0}
\end{gathered}
$$

et aussi

$$
S_{i}^{0}=g^{00} R_{i 0} \quad S_{0}^{0}=\frac{1}{2} g^{00} R_{00}
$$

On en déduit que les équations (26.8) peuvent se mettre sous la forme

$$
g^{00} \partial_{0} S_{\alpha}^{0}=A_{\alpha}^{i \partial_{i}} S_{\lambda}^{0}+B_{\alpha}^{\lambda} S_{\lambda}^{0}
$$

où les $A_{x}^{i \grave{\lambda}}$ et $B_{x}^{\lambda}$ sont des fonctions continues.

Le système des quatre équations (26.9) est linéaire et homogène en $S_{\alpha}^{0}$. Comme $S_{\alpha}^{0}=0$ sur $S$, il n' admet d'autre solution que la solution nulle.

Il résulte ainsi des équations de conservation que, si une solution des équations (26.6) satisfait sur $S$ aux équations (26.7), elle satisfait aussi en dehors de $S$ à ces équations. On traduit ce fait en disant que le système des équations d' EnNsTEIN est en involution.

Nous voyons d'après cette analyse, que le problème initial doit être partage èn denx problèmes distincts:

1. - Problème I ou problème des conditions initiales. Il consiste dans la recherche des données de CAUOHY satisfaisant sur $S$ au système $S_{a}^{0}=0$.

2. - Problème II ou problème de l'évolution. Il consiste dans l'intégration du système $R^{i j}=0$, pour des données de CAUCHY satisfaisant aux conditions du premier problème.

27. Analyse des équations du problème intérieur.

A l'intérieur du domaine $D$ de l'espace-temps, occupé par le fluide, la métrique $d s^{2}$ de l'espace-temps satisfait aux équations

$$
S_{\alpha \beta} \equiv R_{\alpha \beta}-\frac{1}{2} R g_{\alpha \beta}=\chi T_{\alpha \beta}
$$


où

$$
\begin{gathered}
T_{\alpha \beta}=(\rho+p) u_{\alpha} u_{\beta}-p g_{\alpha \beta}-\left(u_{x} q_{\beta}+u_{\beta} q_{\alpha}\right) \\
q_{x}=-x\left(\partial_{\alpha} \theta-Z u_{\alpha}\right) \\
\nabla_{\alpha} q^{\alpha}=c \rho u^{\alpha} \partial_{\alpha} \theta-\frac{l}{\rho} u^{\alpha} \partial_{\alpha \rho} \\
\rho=\varphi(p, \theta) .
\end{gathered}
$$

A ces équations, il faut ajouter les conditions de conservation, ainsi que le caractère unitaire du vecteur vitesse d'univers $u^{x}$.

$$
\begin{aligned}
\nabla_{\alpha} T^{\alpha \beta} & =0 \\
g_{\alpha \beta} u^{\alpha} u^{\beta} & =1 .
\end{aligned}
$$

Ces conditions conduisent à l'équation de continuité et aux équations de mouvement :

$$
\begin{gathered}
\nabla_{\alpha}\left[(\rho+p) u^{\alpha}\right]-u^{\alpha} \partial_{\alpha} p=\nabla_{\alpha} q^{\alpha}-q_{\rho} u^{\alpha} \nabla_{\alpha} u^{\rho} \\
(\rho+p) u^{\alpha} \nabla_{x} u^{\beta}-\partial_{\alpha} p\left(g^{\alpha \beta}-u^{\alpha} u^{\beta}\right)=\nabla_{\boldsymbol{x}}\left(u^{\alpha} q^{\beta}+u^{\beta} q^{\alpha}\right)-u^{\beta}\left(\nabla_{x} q^{\alpha}-q_{\rho} u^{\alpha} \nabla_{x} u^{\rho}\right) .
\end{gathered}
$$

Les coefficients calorifiques $x, c, l$ sont des fonctions connues

$$
\begin{aligned}
& x=x(p, \theta) \\
& c=c(p \cdot \theta) \\
& l=l(p \cdot \theta) .
\end{aligned}
$$

L'exposé des équations montre qu'il y a deux problèmes qui se distin. guent nettement :

1. - la détermination de la structure géométrique de l'espace-temps par les potentiels de gravitation $g_{\alpha \beta}$;

2. - l'étude de l'évolution du schéma matériel, par la détermination des quantités $\rho, p, \theta, u^{\lambda}$.

Nous supposons que l'hypersurface $S$ est localement représentée par l'équation

$$
x^{0}=0 \text {. }
$$

En ce qui concerne le premier problème, les considérations faites au $\$ 26$ précédent, s'appliquent à quelques modifications près. Il nous faut donner sur l'hypersurface $S$ portant les conditions initiales, les potentiels de gravitation $g_{\alpha \beta}$ et leurs dérivées premières d'indice $1, \partial_{0} g_{\alpha \beta}$. Et nous mettons en évidence les dérivées d'indice 2 des $g_{x \beta}$ dans le premier membre des équations d'Einstein prises sous la forme

$$
R_{\alpha \beta}=S_{\alpha \beta}-\frac{1}{2} S g_{\alpha \beta}
$$


soit :

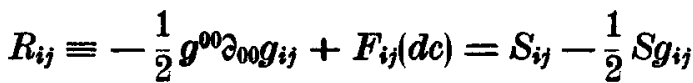

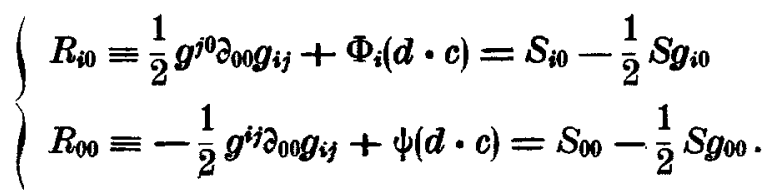

Moyennant les données initiales, si $g^{00} \neq 0$, les équations (27.9a) fournissent les valeurs sur $S$ des dérivées d'indice 2 des potentiels $\partial_{00} g_{i j}$, si l'on connaît les valeurs des seconds membres (c'est-à-dire des quantités $\rho, p, \theta, \partial_{\alpha} \theta, u^{\alpha}$ ).

Ce calcul ne fait intervenir que le groupe des équations $(28.9 a)$, à l'exclusion des équations (27.9b). Or la donnée des $g_{\alpha \beta}$ et $\partial_{0} g_{\alpha \beta}$ sur $S$, definit entièrement les valeurs sur $S$ des quatre quantités $S_{\alpha}^{0}$, comme on a vu au paragraphe précédent. Nous pouvons alors substituer aux équations (27.9b) les equations suivantes

$$
S_{\alpha}^{0}=\chi T_{\alpha}^{0}
$$

qui fournissent le moyen de calculer les quantites $p, \rho, u^{\lambda}$, si nous connaissons $\theta$ et $\partial_{x} \theta$.

Ceci étant dit, l'étude du schéma matériel exige la détermination du champ de température $\theta$. Comme $\theta$ figure dans nos équations (27.2) (27.4) (27.5) par ses dérivées jusqu' à l'ordre 2 , il nous faudra donner les valeurs sur $S$ du champ de température $\theta$ et de sa dérivée d'indice $1: \partial_{0} \theta$, car les dérivées tangentes $\partial_{i} \theta$ et $\partial_{i 0} \theta$ s'obtiennent par simple dérivation. La seule dérivée de $\theta$ qui puisse être discontinue à la traversée de $S$, est la dérivée d'indice $2: \partial_{00} \theta$.

Cette dérivée $\partial_{00} \theta$ figure d'abord dans l'equation de conduction (27.2), puis dans les conditions de conservation (27.4), en l'espèce l'équation de continuité (27.6) et les équations de mouvement (27.7). Sa détermination est donc liée à celle des dérivées de $\rho, p, u^{\lambda}$.

L'analyse précédente nous montre que notre problème se pose sous la forme suivante :

Le problème intérieur. - Etant donnés sur une hypersurface $S$ :

a) le champ de gravitation par ses potentiels $g_{\alpha \beta}$ et leurs dérivées $\partial_{\lambda} g_{\alpha \beta}$,

b) le champ de température par $\theta$ et $\partial_{\lambda} \theta$,

déterminer ces deux champs $\left(g_{\alpha \beta}, \theta\right)$ au voisinage de l'hypersurface $S$.

Nous supposons, comme il a été dit dans, la partie axiomatique, les hypothèsess de différentiabilité suivantes:

$g_{\alpha \beta}$ de classe $\left(C^{1}, C^{3}\right.$ par morceaux $)$.

$\theta$ de classe $\left(C^{2}, C^{\prime}\right.$ par morceaux $)$.

Il nous suffit d'étudier la possibilité de calculer sur $S$ les valenrs des différentes quantités introduites et de leurs dérivées successives. 
28. Détermination de $p, u^{\lambda}, \partial_{v 0} g_{i j}$.

La détermination de $p, u^{\lambda}, \partial_{n} g_{i j}$, se fait à l'aide des équations d'Ers. STEIN (27.1), de l'equation d'état (27.3), auxquelles il convient d'ajouter le caractère unitaire du vecteur vitesse $u^{\lambda}$. Or, nous avons vu que le système des équations d' Ernstein est équivalent à l'ensemble des deux systèmes $(27.9 a)$ et $(27.10)$,

Nous écrivons done les équations

$$
\begin{aligned}
& R_{i j} \equiv-\frac{1}{2} g^{n 0} \partial_{00} g_{i j}+F_{i j}(d c)=\chi\left[(\rho+p) u_{i} u_{j}-\frac{1}{2}(\rho-p) g_{i j}-\left(u_{i} q_{j}+u_{j} q_{i}\right)\right] \\
& S_{\lambda}^{0}=\chi\left[(\rho+p) u^{0} u_{\lambda}-p g_{\lambda}^{0}-\left(u^{0} q_{\lambda}+u_{\lambda} q^{0}\right)\right] \\
& \rho=\varphi(\boldsymbol{p}, \theta) \\
& g^{\lambda}{ }^{\lambda} u_{\lambda} u_{\mu}=1 \text {. }
\end{aligned}
$$

Supposant $p$ provisoirement connu, nous nous proposons d'evaluer en fonction de $p$, les inconnues $u^{0}$ et $Z=u p \partial_{\rho} \theta$. Pour cela, nous écrivons (28.2) sous la forme:

$$
S_{\lambda}^{0}+\chi p g_{\lambda}^{0}=\chi\left[(\rho+p) u^{0} u_{\lambda}-\left(u^{0} q_{\lambda}+u_{\lambda} q^{0}\right)\right] .
$$

Ces équations donnent, si $\nabla^{\prime} \neq 0$

$$
u_{\lambda}=\frac{\left(S_{\lambda}^{0}+\chi p g_{\lambda}^{0}\right)-\chi \chi u^{c} \partial_{\lambda} \theta}{\chi^{0}}
$$

où nous avons posé

$$
V^{0}=(\rho+p-x Z) u^{0}+x\left(\partial^{0} \theta-Z u^{0}\right) .
$$

Nous formons deux équations par multiplication contractée de (28.2') par $g^{20}$ et $\partial^{2} \theta$ respectivement.

$$
\begin{gathered}
S^{00}+\chi p g^{00}=\chi\left[(\rho+p-2 x Z) u^{0}+2 \chi \partial^{0} \theta\right] u^{0} \\
\partial^{\alpha} \theta\left(S_{\lambda}^{0}+\chi p g_{\lambda}^{0}\right)=\chi\left\{\left[(\rho+p-2 \chi Z) u^{0}+\chi \partial^{0} \theta\right] Z+\chi \Delta_{1} \theta u^{0}\right\} .
\end{gathered}
$$

En introduisant $\nabla^{0}$, nous donnons aux deux équations établies, la forme

$$
\begin{aligned}
\chi\left(\nabla^{0}+\chi \partial^{0} \theta\right) u^{0} & =\chi P^{00}(p) \equiv S^{00}+\chi p g^{00} \\
\chi\left(V^{0} Z+\chi \triangle_{1} \theta u^{0}\right) & =\chi Q^{\prime}(p) \equiv\left(S^{0 \lambda}+\chi p g^{0 \lambda} ; \partial_{\lambda} \theta\right.
\end{aligned}
$$

où $\triangle_{1} \theta=g^{\lambda \mu} \partial_{\lambda} \theta \partial_{\mu} \theta$.

Les deux équations (28.6), $(28,7)$ déterminent linéairement $u^{0}$ et $Z$ à partir de $\nabla^{0}$ ou d'une inconnue équivalente. Or, en exprimant à partir de $\left(27.2^{\prime}\right)$ le caractère unitaire de $u_{\lambda}$ il vient

$$
\begin{gathered}
\chi^{2} g^{\lambda \mu}\left[(\rho+p) u^{0} u_{\lambda}-\left(u^{0} q_{\lambda}+u_{\lambda} q^{0}\right)\right]\left[(\rho+p) u^{0} u_{\mu}-\left(u^{0} q_{\mu}+u_{\mu} q^{v}\right)\right] \\
=\chi^{2}\left[R^{0}(p)\right]^{2} \equiv g^{\lambda \mu}\left(S_{\lambda}^{0}+\chi p g_{\lambda}^{0}\right)\left(S_{\mu}^{0}+\chi p g_{\mu}^{0}\right) .
\end{gathered}
$$


Le premier membre se calcule facilement. Il est égal à :

$$
\left[(\rho+p) u^{0}-q^{0}\right]^{2}+\left(u^{0}\right)^{2} g^{\lambda \mu} q_{\lambda} q_{\mu} \text {. }
$$

Or, $q^{0}=-x\left(\partial^{\circ} \theta-Z u^{0}\right)$ et $g^{\lambda \mu} q_{\lambda} q_{\mu}$ est le carré du vecteur $q_{\lambda}$. Ce vecteur est la composante d'espace du vecteur $-x \operatorname{grad} \theta$. La composante de temps de ce dernier est $-x Z u_{\lambda}$. On en déduit par le théorème de Pythagone:

$$
g^{\lambda \mu} q_{\lambda} q_{\mu}=x^{2}\left(\triangle_{1} \theta-Z^{2}\right)
$$

Il vient alors l'équation

$$
\chi^{2}\left\{\left[(\rho+p) u^{0}+x\left(\partial^{0} \theta-Z u^{0}\right)\right]^{2}+x^{2}\left(\triangle_{1} \theta-Z^{2}\right)\left(u^{0}\right)^{2}\right\}=\chi^{2}\left[R^{0}(p)\right]^{2} .
$$

En introduisant $\nabla^{0}$, elle s' écrit :

$$
\left(\nabla^{0}\right)^{2}+2 x Z \nabla^{0} u^{0}+x^{2} \triangle_{1} \theta\left(u^{0}\right)^{2}=\left[R^{0}(p)\right]^{2} .
$$

Nous éliminons ensuite $u^{0}, Z$ du système des trois équations (28.6) (28.7) (28.8), ce qui conduit à l'équation

$$
\left(\nabla^{0}\right)^{2}\left(V^{0}+x \partial^{0} \theta\right)^{2}-\left(R^{0}\right)^{2}\left(V^{0}+x \partial^{0} \theta\right)^{2}+2 x P^{00} Q^{0}\left(V^{0}+x \partial^{0} \theta\right)-x^{2} \triangle_{1} \theta\left(P^{\circ 0}\right)^{2}=0 .
$$

En substituant à $\nabla^{0}$, l'inconnue

$$
W^{0}=V^{0}+x \partial^{0} \theta
$$

nous obtenons finalement l'équation :

(28.10) $\left(W^{0}\right)^{4}-2 x \partial^{0} \theta\left(W^{0}\right)^{3}+\left[x^{2}\left(\partial^{0} \theta\right)^{2}-\left(R^{0}\right)^{2}\right]\left(W^{0}\right)^{2}+2 x P^{00} Q^{0}\left(W^{0}\right)-x^{2} \triangle_{1} \theta\left(P^{00}\right)^{2}=0$.

L'équation précédente définit $W^{\circ}(p)$. Nous en déduisons $V^{\circ}(p)$, puis $p$ ̀े l'aide des relations (28.3), (28.5) et (28.6).

En portant les valeurs ainsi calculées de $p, u_{\lambda}$ dans les équations (28.1); nous déterminerons finalement les valeurs des dérivées d'indice 2: $\partial_{00} g_{i j}$.

29. Détermination de $\partial_{0} p, \partial_{0} u^{\lambda}, \partial_{00} \theta$.

La détermination des dérivées $\partial_{0} p, \partial_{0} u^{\lambda}, \partial_{00} \theta$ s'effectue à l'aide de l'équa. tion de conduction thermique (27.2) et des conditions da conservation imposées au tenseur d'impuision-énergie. Si on tient compte du caractère unitaire de $\vec{u}$, ces conditions de conservation conduisent à l'équation de continuite et aux équations de mouvement. Il en résulte que nous disposons du système

$$
\begin{gathered}
\nabla_{\alpha} q^{\alpha}=c \rho u^{\alpha} \partial_{\alpha} \theta-\frac{l}{\rho} u^{\alpha} \partial_{\alpha \rho} \\
\nabla_{\alpha}\left[(\rho+p) u^{\alpha}\right]-u^{\alpha} \partial_{\alpha} p=\nabla_{\alpha} q^{\alpha}-q_{\rho} u^{\alpha} \nabla_{\alpha} u^{\rho} \\
(\rho+p) u^{\alpha} \nabla_{\alpha} u^{\beta}-\partial_{\alpha} p\left(g^{\alpha \beta}-u^{\alpha} u^{\beta}\right)=\nabla_{\alpha}\left(u^{\alpha} q^{\beta}+u^{\beta} q^{\alpha}\right)-u^{\beta}\left(\nabla_{\alpha} q^{\alpha}-q_{\rho} u^{\alpha} \nabla_{\alpha} u \rho\right) .
\end{gathered}
$$


Introduisant encore $Z$ comme inconnue auxiliaire, nous formons ane équation supplémentaire par multiplication contractée de $(29,3)$ par $\partial_{\beta} \theta$, soit :

$$
(\rho+p) u^{\alpha} \nabla_{x} u^{\beta} \partial_{\beta} \theta-\partial_{\alpha} p\left(\partial^{\alpha} \theta-Z u^{\alpha}\right)=\partial_{\beta} \theta \nabla_{\alpha}\left(u^{\alpha} q^{\beta}+u^{\beta} q^{\alpha}\right)-Z\left(\nabla_{\alpha} q^{\alpha}-q_{\rho} u^{\alpha} \nabla_{\alpha} u \rho\right)
$$

La connaissance de $p, u^{\lambda}, \partial_{0} \theta$ sur $S$ calculées au paragraphe précédent, entraìne celle des dérivées tangentes $\partial_{i} p$, $\partial_{i} u^{\lambda}$, et $\partial_{i 0} \theta$. En tenant compte de l'équation d'état $\rho=\varphi(p, \theta)$ et mettant en évidence les inconnues, nous obtiendrons le-système des équations:

$$
\begin{gathered}
\frac{l}{\rho} u^{0} \frac{\partial \varphi}{\partial p} \partial_{0} p+x Z \partial_{0} u^{0}-x g^{00} \partial_{00} \theta+x u^{0} \partial_{0} Z=H_{1}(d c) \\
u^{0} \frac{\partial \varphi}{\partial p} \partial_{0} p+(\rho+p-x Z) \partial_{0} u^{0}+x\left[g^{00}+\left(u^{0}\right)^{2}\right] \partial_{00} \theta-2 x u^{0} \partial_{0} Z=H_{2}(d c) \\
{\left[g^{00}-\left(u^{0}\right)^{2}\right] \partial_{0} p-\left(W^{0}-x Z u^{0}\right) \partial_{0} u^{0}-x\left[g^{00}-\left(u^{0}\right)^{2}\right] u^{0} \partial_{00} \theta=H_{3}(d c)} \\
\left(\partial^{0} \theta-Z u^{0}\right) \partial_{0} p-x\left(\triangle_{1} \theta-Z^{2}\right) \partial_{0} u^{0}+(\rho+p-x Z)\left(u^{0}\right)^{2} \partial_{00} \theta-V^{0} \partial_{0} Z=H_{4}(d c)
\end{gathered}
$$

et les trois équations

$$
\left(g^{0 i}-u^{0} u^{i}\right) \partial_{0} p-x\left(\partial^{i} \theta-Z u u^{i}\right) \partial_{0} u^{0}-V^{0} \partial_{0} u^{i}-x u^{i}\left(g^{0 i}-u^{0} u^{i}\right) \partial_{00} \theta=K^{i}(d c)
$$

où les $H_{x}$ et $K^{i}$ sont des expressions calculables sur $S$ à partir des données de CAUCHY, par opérations algébriques ou par dérivation sur $S$.

Nous voyons que si $V^{0} \neq 0$, les $\partial_{0} u^{i}$ sont immédiatement connues quand les autres inconnues on êté évaluées.

Les quantités $\partial_{n} p, \partial_{0} u^{0}, \partial_{00} \theta, \partial_{0} Z$ sont parfaitement déterminées par le système des quatre équations (29.5) (29.6) (29.7) et (29.8) qui sont linéaires par rapport aux inconnues, pourvu que le déterminant $D$ du système soit différent de zéro.

Ainsi, si

$$
\nabla^{0} \neq 0 \quad D \neq 0
$$

on peut calculer les dérivées $\partial_{0} p, \hat{\partial}_{0} u^{\lambda}, \partial_{00} \theta_{\text {. }}$

30. Caractère général de la solution du problème intérieur.

Les calculs des deux paragraphes précédents (\$§ 28 et 29) montrent que si les données de. CAUCHY $\left(g_{\alpha \beta}, \partial_{0} g_{\alpha \beta} ; \theta, \partial_{0} \theta\right)$ sont respectivement trois fois et deux fois continúment dérivables sur l'hypersurface $S$ qui les porte, les équations (28.2) (28.3) fournissent les valeurs de $p$, $u^{\lambda}$ sur $S$, les équations (28.1) fournissent les valeurs de $\partial_{00} g_{i j}$ et les équations (29.1) (29.2) (29.3) fournissent les valeurs de $\partial_{0} p, \partial_{0} u^{\lambda}, \partial_{00} \theta$. 
Si l'hypersurface $S$ satisfait aux trois hypothèses

$$
g^{00} \neq 0 \quad \nabla^{0} \neq 0 \quad D^{0} \neq 0
$$

les diverses quantites $\left(p, u^{\lambda}, \partial_{00} g_{i j}, \partial_{0} p, \partial_{0} u^{\lambda}, \partial_{00} \theta\right)$ ont sur $S$ des valeurs bien déterminées et ne peuvent pas ètre discontinues à la traversée de $S$.

Si les données de CAUCHY sont dérivables localement à un ordre supé. rieur, les mêmes conclusions s'étendent aux dérivées d'ordre supérieur sur $S$ de ces quantités. Il suffit de dériver par rapport à $x^{0}$, les équations (28.1) pour avoir les dérivées d'indice supérieur de. $g_{i j}$, les équations (29.1) (29.2) et (29.3) pour avoir les dérivées $d^{\prime}$ indice supérieur de $\left(p, u^{\lambda}, \theta\right)$. On remarque que cette détermination s' effectue alternativement entre les deux groupes. Elle traduit en fait la séparation logique du champ de gravitation et du schéma matériel, en même temps que leur interaction.

Ceci étant dit, si nous posons

$$
Q_{\beta}^{\alpha} \equiv S_{\beta}^{\alpha}-\chi T_{\beta}^{\alpha}
$$

les équations d'Ernsterr qui s'étaient partagées en deux groupes, vont s'écrire:

$$
\begin{aligned}
& Q_{i j} \equiv R_{i j}-\chi\left(T_{i j}-\frac{1}{2} T g_{i j}\right)=0 . \\
& Q_{\lambda}^{0} \equiv R_{\lambda}^{0}-\chi\left(T_{\lambda}^{0}-\frac{1}{2} T g_{\lambda}^{0}\right)=0 .
\end{aligned}
$$

Les tenseurs $S^{\alpha \beta}$ et $T^{\alpha \beta}$ sont conservatifs, il en résulte que le tenseur $Q^{\alpha \beta}$ est aussi conservatif :

$$
\nabla_{\alpha} Q_{\beta}^{\alpha}=0 .
$$

Nous allons montrer que les premiers membres des équations (30.3) et (30.4) sont lies par des relations provenant des conditions de conservation de $Q_{\beta}^{\alpha}$.

En effet, si nous considérons une solution $\left(g_{\alpha \beta}, \theta ; p, u^{\lambda}\right)$ du problème intérieur, un ealeul donne:

$$
\begin{aligned}
& Q_{i}^{0}=g^{00}\left[R_{i 0}-\chi\left(T_{i 0}-\frac{1}{2} g_{0 i} T\right)\right] \\
& Q_{0}^{0}=\frac{1}{2} g^{00}\left[R_{00}-\chi\left(T_{00}-\frac{1}{2} g_{00} T\right)\right] .
\end{aligned}
$$

Il en résulte que la quantité

$$
R+\chi T=g^{\alpha \beta}\left[R_{\alpha \beta}-\chi\left(T_{\alpha \beta}-\frac{1}{2} g_{\alpha \beta} T\right)\right]
$$


s'exprime par une combinaison linéaire et homogène à coefficients continus $\operatorname{des} Q_{x}^{0}$. Par suite, il en est de mème des composantes:

$$
Q_{\beta}^{i}=g^{\alpha i} Q_{\alpha \beta}=g^{\alpha i}\left[R_{\alpha \beta}-\chi\left(T_{\alpha \beta}-\frac{1}{2} g_{\alpha \beta} T\right)\right] .
$$

Il s'ensuit que les conditions de conservation (30.5) qui s'écrivent:

$$
\nabla_{a} Q_{\beta}^{x}=\nabla_{0} Q_{\beta}^{0}+\nabla_{i} Q_{\beta}^{i}=0
$$

se réduisent, pour une solution des équations (30.3) a :

$$
g^{00} \partial_{0} Q_{\beta}^{0}=A_{\beta}^{i \alpha} \partial_{i} Q_{x}^{0}+B_{\beta}^{\alpha} Q_{x}^{0}
$$

où les $A_{\beta}^{i_{\alpha}}$ et $B_{\beta}^{\alpha}$ sont des fonctions continues.

Le système des quatre equations $(30.6)$ est linéaire et homogène, en $Q_{\beta}^{0}$. Comme $Q_{\beta}^{0}=0 \operatorname{sur} S$, il n'admet d'autre solution en dehors de $S$ que la solution identiquement nulle. Il en résulte que si une solution du problème satisfait sur $S$ aux equations (30.4), elle satisfait aussi en dehors de $S$, à ces equations.

I.'étude précédente précise le caractère de la solution du problème inté. rieur. En effet, notre problème initial se trouve partagé en deux problèmes:

1. - Problème I. - Recherche des données de CAUCHY $\left(g_{a \beta}, \partial_{0} g_{\alpha \beta} ; \theta, \partial_{0} \theta\right)$ rendant compatibles les équations

$$
\begin{gathered}
S_{\lambda}^{0}=\chi T_{\lambda}^{0} \\
g^{\lambda \mu} u_{\lambda} u_{\mu}=1
\end{gathered}
$$

et détermination à l'aide de ces données, des quantités $p, u_{\lambda}$.

2. - Proolème II. - Intégration, pour des données de CaUCHY ainsi choisies, du système des équations

$$
\begin{gathered}
R_{i j}=S_{i j}-\chi T_{i j} \\
\nabla_{\alpha} q^{\alpha}=c \rho u^{\alpha} \partial_{\alpha} \theta-\frac{l}{\rho} u^{\alpha \partial_{\alpha} \rho} \\
\nabla_{\alpha} T^{\alpha \beta}=0 .
\end{gathered}
$$

Supposons un instant, ce qui est d'ailleurs contraire aux axiomes que nous avons posés aux chapitres $I$ et II, que toutes. les données da problème soient analytiques réelles. On peut alors établir, à l'aide du théorème de CaUdhY-Kowaleska pour les équations aux dérivées partielles, qu'à un changement de coordonnées près, conservant point par point l'hypersurface $S$ et les valeurs des données de CAdorY sur $S$, le problème admet une solution analytique réelle et une seule. Mais en fait l'analycité ne doit jouer aucun rôle ici. Ce résultat nous montre seulement la voie à suivre. 


\section{II. - Le problème de raccordement.}

\section{Génóralités.}

On se propose de représenter sur une variété espace-temps $V_{4}$, un modèle comportant plusieurs domaines meublés par de la matière. Dans chacun de ces domaines meublés, la matière engendre un tube d'univers limité par une hypersurface $S$. A l'intérieur de $S$, la métrique de l'espacetemps doit satisfaire aux équations d'EINsTEIN du cas intérieur :

$$
S_{\alpha \beta}=\chi T_{\alpha \beta} \text {. }
$$

A l'extérieur de tous les domaines meublés, elle doit vérifier les équations d' Einstein du cas extérieur

$$
S_{\alpha \beta}=0 \text {. }
$$

Dans chacun de ces domaines, les potentiels $g_{\alpha \beta}$ relatifs à un système de coordonnées admissible, ainsi que leurs dérivées premières sont continues. Pour ce qui se passe à la traversée de $S$ conformément aux axiomes géné. raux de la relativité générale (\$ 1), nous devons imposer les conditions suivantes, dites conditions de raccordement de SchWarzoHILD.

Les conditions de vecordement. - Pour tout point $x$ de $S$, il existe un système de coordonnées admissibles dont le domaine contient $x$, tel que les potentiels relatifs à ce système et leurs dérivées premières soient continus à la traversée de $S$.

Ces conditions sont relatives aux potentiels de gravitation.

Etant donnée la structure des équations du schéma matériel, nous imposons également des conditions analogues au champ de température.

Nous supposerons done que les quantités

$$
g_{\alpha \beta}, \partial_{\lambda} g_{\alpha \beta}, \theta, \partial_{\lambda} H
$$

sont continues à la traversée des hypersurfaces de raccordement.

L'étude du problème de raccordement présente ici un caractère local. On peut prendre pour coordonnées admissibles, les coordonnées de Gauss.

Etant donnée une hypersurface $S$ de $\nabla_{4}$, les coordonnées de Gauss relatives à $S$ sont définies de la manière suivante. Supposons que l'hypersurface $S$ soit définie localement par l'équation

$$
x^{0}=0,
$$

chaque point de $S$ par ses coordonnées $x^{i^{\prime}}$. Les coordonnées $\left(x^{x^{\prime}}\right)$ sont choisies de façon que les lignes sur lesquelles la variable $x^{\prime \prime}$ varie seule, les $x^{{ }^{\prime \prime}}$ restant constantes, soient les géodésiques issues des points $x^{\prime \prime} \in S$ et normalement à $S$. 
Si $\vec{\nabla}$ est le vecteur normó, tangent aux géodésiques, il existe localement une fonction $\varphi$ telle que $\left({ }^{4}\right)$

$$
\nabla_{\alpha}=\partial_{a} \varphi \text {. }
$$

Au voisinage de $S$, la métrique de $\nabla_{4}$ peut prendre alors la forme

où $x^{\prime \prime}=\varphi$ et $x^{i}=x^{i}$.

$$
d s^{2}=g_{00}\left(d x^{0}\right)^{2}+g_{i j}\left(x^{\lambda}\right) d x^{i} d x^{j}
$$

$g_{00}=+1$ si $S$ est orientée dans l'espace, et $g_{00}=-1$ si $S$ est orientée dans le temps.' Nous verrons que l'hypersurface de raccordement doit etre orientée dans le temps.

\section{Prolongement de l'intérieur de la matière vers l'extérieur.}

Donnons nous, dans une région limitée à une hypersurface $S$, un $d s^{2}$ intérieur correspondant $\dot{a}$ un schéma fluide parfait thermodynamique. Et proposons-nous de rechercher à quelle condition il existe, de l'autre coté de $S$, un $d s^{2}$ extérieur se raccordant le long de $S$ avec le $d s^{2}$ intérieur donné.

$L$ hypersurface $S$ étant définie localement dans un système de coordon. nées admissibles, par l'équation

$$
x^{0}=0
$$

nous supposons qu'il existe un $d s^{2}$ extérieur répondant à la question. Les $S_{\lambda}^{0}$ associées à ce $d s^{2}$ extérieur sont identiquement nulles. Or, à l'intérieur de $S$ et $\operatorname{sur} S$, les $S_{\lambda}^{0}$ ne dépendent que des potentiels $g_{x B}$, de leurs dérivées premières et de leurs dérivées secondes d'indice 1 (cf. $\$ 26$ ). En vertu des conditions de raccordement, les quantités $S_{r}^{0}$ associées au $d s^{2}$ intérieur doivent être nulles sur $S$.

Il faut done que l'on ait sur $S$ :

$$
T_{\lambda}^{0}=(\rho+p) u^{0} u_{\lambda}-p g_{\lambda}^{0}-\left(u^{0} q_{\lambda}+u_{\lambda} q^{0}\right)=0 .
$$

D'autre part, le raccordement du champ d'un fluide parfait thermodyna. mique avec un champ extérieur doit s'effectuer le long d'une hypersurface $S$ sur laquelle

$$
\theta=\text { const } \quad \partial^{*} \theta=0 .
$$

I) en résulte que $q^{\lambda}=0$ sul $^{2} S$.

De (32.1), nous tirons

$$
(\rho+p) u^{0} u_{\lambda}-p g_{\lambda}^{0}=0 .
$$

En exprimant le caractère unitaire de $u_{\lambda}$, on trouve $\rho u^{0}=0$. On en déduit $u^{0}=0$, puis $p=0$.

Ainsi nous avons nécessairement sur $S$ :

$$
u^{0}=0 \quad p^{0}=0
$$

(4) A. Lichnerowicz, Cours du Collège de France, 1952-1953, chapitre III, [18]. 
Autrement dit, l'hypersurface de raccordement $S$ est engendrée par les lignes de courant du schéma intériour. La pression $p$ s'annule sur $S$.

Réciproquement, supposons qu'il en soit ainsi sur $S$. Les quantités $S_{\text {. }}^{0}$ associées an $d s^{*}$ intérieur sont nulles. L'hyporsurface $S$ engendrée par les lignes de courant, est partout orientée dans le temps. Un $d s^{2}$ extérieur se raccordant avec le $d s^{2}$ intérieur donné, est alors une solution du problème de CAUCHY extérieur, relatif à $S$ et aux données précédentes. Nous savons que ce problème a localement une solution bien déterminée (cf. $\$ 26$ ).

Nous pouvons done énoncer.

THGoRime. - La condition nécessaire et suffisante pour que le problème du prolongement de l'intérieur vers l'extérieur admette une solution locale - et cette salution est unique - est que:

1. - l'hypersurface $S$ soit engendrée par les lignes de courant du champ intérieur,

2. - la pression $p$ s'annule sur $S$.

33. Prolongement de l'extérieur vers l'intérieur.

Un $d s^{2}$ extérieur limité à uqe hypersurface $S$ étant donné, nous nous proposons de rechercher s'il existe un $d s^{2}$ intérieur correspondant au schéma fluide parfait thermodynamique, qui se raccorde avec le $d s^{2}$ extérieur le long de $S$. Il est entendu que sur $S$ :

$$
\theta=\text { const } \quad \partial^{\circ} \theta=0 .
$$

Il en résulte que $q_{\lambda}=0$. Comme sur $S$, nous devons avoir $S_{\lambda}^{0}=0$, nous déduisons que:

$$
(\rho+p) u^{0} u_{\lambda}-p g_{\lambda}^{0}-\left(u^{0} q_{\lambda}+u_{\lambda} q^{0}\right)=0
$$

et $u^{0}=0, p=0$ ce qui entraîne

$$
V^{0} \equiv(p+p-x Z) u^{0}+x\left(\partial^{0} \theta-Z u^{0}\right)=0
$$

et nous sommes dans l' un des cas exceptionnels où le problème intérieur n'est pas correctement posé : ce problème de CAUCHY intérieur est impossible, ou bien il admet plusieurs solutions.

On peut interpréter cette circonstance en disant qu' un champ extérieur donné peut ne pas être compatible avec une distribution effective d'energie matérialisée sous la forme de fluide parfait thermodynamique, sinon peut l'être avec plusieurs telles distributions.

Cette conclusion permet d'étendre eertains résultats connus en théorie de gravitation concernant la régularité des $d s^{2}$.

En particulier, si on considère dans un champ de gravitation extérieur donné, un tube d'univers infiniment mince représentant une très petite masse d'épreuve, les interactions internes sont négligeables; et le champ intérieur du tube peut être considéré comme un schéma matière pure. Le principe des géodésiques doit être conservé. 


\section{III. - Etude des variétés exceptionnelles.}

\section{Généralités sur les variótés exceptionnelles.}

An cours de l'étude du problème de CAUCHY rèlatif à un fluide parfait thermodynamique, nous avons va que le problème n'est par déterminé, si l'hypersurface $S$ d'equation locale $x^{0}=0$, qui porte les données de CAdCHY est telle que:

$$
g^{00}=0, \quad V^{0}=0 \quad \text { ou } \quad D=0 .
$$

De telles variétés $S$ seront dites les variétés exceptionneltes du problème de Cauchy.

Que se passe-t-il à la traversée de ces variétés?

Si $g^{00}=0$, les dérivées d'indice 2 des potentiels de gravitation $\partial_{00} g_{i j}$ sont discontinues à la traversée de $S$. Il peut exister une infinité de solutions distinctes des équations d'Einstern correspondant aux mêmes données de CAUOHY sur $S$. La variété $S$ est tangente en chacun de ses points, au cône élémentaire $\Gamma_{x}$ associé à ce point (cf. § 2). Or les cônes $\Gamma_{x}$ sont les cônes caractéristiques du système des équations d'EINSTEIN aussi bien intérieur qu' extérieur.

Les variétés telles que $g^{00}=0$ sont les variétés caractéristiques. Elles seront appelées $V_{\mathbf{3}}^{c}$.

Les variétés exceptionnelles $\nabla^{0}=0$ correspondent au problème de la détermination algébrique des quantités $p, u^{\lambda}$,. . A la traversée de ces variétes, la pression, la densité et le vecteur vitesse unitaire du fluide peuvent pré. senter des discontinuités.

A la traversée des variétés telles que $D=0$, ce sont les dérivées des caractéristiques du fluide: $\partial_{0} p, \partial_{0} u^{\lambda}, \partial_{0} q^{\lambda}$ qui peuvent présenter des discontinuités.

Ces variétés constituent donc l'extension relativiste des surfaces d'ondes considerées comme surfaces de discontinuités au sens d'Hugoniot-RiEmanN.

Les variétés $\nabla^{0}=0$ seront appelées les variétés exceptionnelles d'ordre 0 ou les $\nabla_{3}^{0}$. Les variétés $D=0$ seront appelées les variétés exceptionnelles du ler ordre ou les $\nabla_{3}^{H}$.

Dans un système de coordonnées arbitraire, l'hypersurface $S$ aura pour équation :

$$
f\left(x^{\lambda}\right)=0 .
$$

Pour que la surface de discontinuité qu' elle représente, ait une signification physique, il faut faire l'hypothèse qu'elle soit orientée dans le temps, ou à la limite, tangente aux oônes elémentaires.

Cette hypothèse se traduit par l'inégalité

$$
\triangle_{1} f \equiv g^{\lambda \mu} \partial_{\lambda} f \partial_{\mu} f \leq 0
$$

Nous verrons qu' elle satisfait aux exigences relativistes de la théorie. 
35. Les vuriétés caractériques $V_{3}^{c}$.

Si $S$ est représentée localement par l'équation $f\left(x^{\alpha}\right)=0$, les variétés caractéristiques sont les solutions. de l'équation aux dérivées partielles du ler ordre :

$$
\triangle_{1} f \equiv g^{\alpha \beta} \partial_{\alpha} f \partial_{\beta} f=0 .
$$

Les variétés caractéristiques $\nabla_{3}^{c}$ des équations d'Ensstein peuvent ètre engendrées au moyen des multiplicités caractéristiques de l'équation (35.1). Chaque multiplicité caractéristique en est constituée par l'ensemble d'une courbe caractéristique $L_{0}$ et d'éléments de contact attachés à chaque point de cette courbe et tangentes à cette courbe.

On donne aux courbes caractéristiques $L_{0}$ de (35.1), le nom de bicaracté. ristiques des équations d' ErNstein. Nous allons les déterminer.

Désignons par $2 H\left(x^{\lambda}, \partial_{\mu} f\right)$ le premier membre de l'équation (35.1) et posons : $y_{\alpha}=\partial_{x} f . \mathrm{Il}$ vient :

$$
\triangle_{1} f \equiv 2 H\left(x^{\lambda}, \partial_{\mu} f\right)=g^{x \partial_{\alpha}} f \partial_{\beta} f=0 .
$$

Relativement aux variables $x^{x}, f, y_{\beta}$, les bandes caractéristiques de (35.1) sont solutions $d u$ système différentiel :

$$
\frac{d x^{0}}{\frac{\partial H}{\partial y_{0}}}=\ldots=\frac{d x^{3}}{\frac{\partial H}{\partial y_{3}}}=\frac{d f}{2 H}=-\frac{d y_{0}}{\frac{\partial H}{\partial x^{\prime \prime}}}=\ldots=-\frac{\cdot d y_{3}}{\frac{\partial H}{d x^{3}}}=d u
$$

où $u$ désigne une variable auxiliaire. Elles satisfont donc au système différentiel :

$$
\frac{d x^{z}}{d u}=\frac{\partial H}{\partial y_{x}} \quad \frac{d y_{\beta}}{d u}=-\frac{\partial H}{\partial x^{\beta}}
$$

et à l'intégrale première :

$$
2 H\left(x^{\lambda}, y_{\mu}\right)=C \text {. }
$$

On reconnaît dans le système (35.3), le système des équations canoniques de Hamidon relatif à la fonction hamiltonienne $H\left(x^{\lambda}, y_{\mu}\right)$.

En posant

$$
\dot{x}^{a}=\frac{d x^{\alpha}}{d u}
$$

on tire des equations (35.3)

$$
\dot{x}^{\alpha}=g^{\alpha \beta} y_{\beta} \quad \text { ou } \quad y_{\beta}=g_{\alpha \beta} \dot{x}^{\alpha}
$$

Les solutions $x^{\alpha}(u)$ de $(35.3)$ sont alors extremales de la fonction lagrangienne $L$ dófinie par

$$
2 L=g_{\alpha \beta} \dot{x^{\alpha}} \dot{x^{\beta}}
$$


puisque, par passage des variables $\left(x^{x}, \dot{x}^{\beta}\right)$ aux variables $\left(x^{x}, y_{\beta}\right)$, on a entre $H$ et $L$ la relation

$$
H=\dot{x}^{x} \frac{\partial L}{\partial \dot{x}^{x}}-L(=L) \text {. }
$$

Les solutions $x^{x}(u)$ de (35.3) sont donc les extrèmales satisfaisant à l'intégrale première :

$$
2 L=C
$$

pour la valeur $C=0$ de la constante $C$.

Il en résulte que les bicaractéristiques des équations d'Einsmein sont les géodésiques de longueur nulle $d u d s^{2}$. Et les variétés caractéristiques $\nabla_{3}^{\mathrm{c}}$ peuvent être engendrées par les bandes de $\nabla_{4}$ définies par les géodésiques de longueur nulle $L_{0}$, le 3-plan associé étant le plan tangent au còne élémentaire, le long de la tangente à $L_{0}$.

En se reportant à la théorie de propagation par ondes telle qu'elle découle des travaux de HADAMARD, on voit que le champ de gravitation présente, en relativité le caractère d'un phénomène de propagation. Les variétés caractéristiques du champ jouent le rôle de surfaces d'onde gravifique et les bicaractéristiques $L_{0}$, celni des rayons gravifiques $\left({ }^{5}\right)$.

36. Vitesse de propagation des surfaces de discontinuités.

Dans le langage de la théorie de propagation par ondes, les surfaces de discontinuités sont des surfaces d'ondes. La généralisation de l'hypothèse d'Hugoniot permet d'évaluer ce qu'on entend par vitesse de propagation.

Considérons en effet deux surfaces d'ondes voisines définies par les équations

$$
\begin{array}{ll}
\Omega_{0}: & f\left(x^{\alpha}\right)=0 \\
\Omega_{\varepsilon}: & f\left(x^{\alpha}\right)=\varepsilon .
\end{array}
$$

$\varepsilon$ sera pris dans la suite comme infiniment petit principal :

Soit $x$ un point de $\Omega_{0}$. La ligne de courant issue de $x$ coupe la surface d'onde voisine $\Omega_{\varepsilon}$ en un point $x^{\prime}$ défini aux infiniment petits d'ordre supérieur près par

$$
x^{\prime}=x+\eta \vec{u}
$$

où $\eta$ est donné par la relation

$$
\eta u^{\lambda} \partial_{\lambda} f=\varepsilon
$$

Soit $\vec{v}$ le vecteur unitaire normal en $x$ à $\mathbf{Q}_{0}$. Par hypothèse la surface $\mathbf{Q}$ est orientée dans le temps. On a donc $\overrightarrow{(v)}^{2}=-1$. Le vecteur $\vec{v}$ a done pour

(5) G. Darmols [4] et A. Lichnerowicz [18]. 
composantes

$$
\nu_{\lambda}=\frac{\partial_{\lambda} f}{\sqrt{-g^{\lambda \mu} \partial_{\lambda} f \partial_{\mu} f}} .
$$

La trajectoire orthogonale aux surfaces d'onde voisines de $\Omega_{0}$, qui est issue de $x$, est tangente au vecteur $\vec{v}$. Elle coupe $\Omega_{\varepsilon}$ au point defini aux infiniment petits d'ordre supérieur près par

$$
x+\eta_{1} \vec{v}
$$

où $\eta_{1}$ est donné par la relation

$$
\eta_{1} \nu^{2} \partial_{\lambda} f=\varepsilon
$$

Introduisons le vecteur

$$
\vec{t}=\eta \vec{u}-\eta_{1} \vec{v}
$$

Nous avons:

$$
\vec{t} \cdot \vec{v}=\eta(\vec{u} \cdot \vec{v})-\eta_{1}(\vec{v})^{2}=\eta \vec{u} \cdot \vec{v}+\eta_{1}
$$

Or :

$$
\eta_{1}=\frac{-\varepsilon}{\sqrt{-g^{\lambda \mu} \partial_{\lambda} f \partial_{\mu} f}} \quad \vec{u} \cdot \vec{v}=\frac{u^{\lambda} \partial_{\lambda} f}{\sqrt{-g^{\lambda} \mu \partial_{\lambda} f \partial_{\mu} f}} .
$$

En vertu de $(36.1)$ et $(36.3)$, il vient

$$
\vec{t} \cdot \vec{v}=0 \text {. }
$$

Le vecteur $\vec{t}$ est donc tangent à $\Omega_{0}$ en $x$. Soit $\eta_{0}$ son module

$$
\eta_{0}^{2}=(\vec{t})^{2}=\eta^{2}(\vec{u})^{2}+\eta_{1}^{2}(\vec{v})^{2}-2 \eta \eta_{1}(\vec{u} \cdot \vec{v}) .
$$

Il est facile de vérifier que $\eta \vec{u} \cdot \vec{v}=-\eta_{1}$. Il en résulte

$$
\eta_{0}^{2}=\eta_{1}^{2}+\eta^{2} \geq 0
$$

On en déduit que le vecteur $\vec{t}$ est orienté dans le temps.

Le vecteur $\eta \vec{u}$ se trouve ainsi décomposé en une somme de deux vecteurs, une composante orthogonale à la surface d'onde et orientée dans l'espace, l'autre composante tangente à la surface d'onde et orientée dans le temps. La vitesse de propagation de l'onde est définie comme la limite du rapport des modules de ces deux vecteurs, soit :

$$
T=\lim _{i \rightarrow 0} \frac{\left|\eta_{1}\right|}{\left|\eta_{0}\right|}
$$

On en déduit le carré de cette vitesse qui est donné par la formule

$$
T^{2}=-\frac{\left(u^{\lambda} \partial_{\lambda} f\right)^{2}}{\left(g^{\lambda \mu}-u^{\lambda} u^{\mu}\right) \partial_{\lambda} f \partial_{\mu} f}
$$

On remarque au numérateur la grandeur de temps du vecteur gräd $f$ et au dénominateur sa grandeur d'espace. 
Pham MaU Quan: Sur une théorie relatiriste des fluides, etc.

181

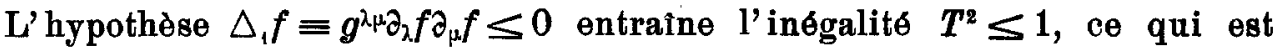
conforme aux exigences relativistes.

Dans le système de coordonnées locales où la surface d' onde est définie par $x^{0}=$ const, nous avons la formule :

$$
T^{2}=-\frac{\left(u^{0}\right)^{2}}{g^{00}-\left(u^{0}\right)^{2}} \quad \text { où } \quad g^{00} \leq 0 .
$$

37. Les variétés exceptionnelles du premier ordre $\nabla_{3}^{H}$.

Elles se présentent dans l'étude du schéma thermodynamique. A la traversée de ces variétés $\nabla_{3}^{H}$, se produisent des discontinuités du gradient de pression (ainsi que des quantités $\partial_{0} \rho, \partial_{0} u^{\lambda}, \partial_{0} q^{\lambda}$ ). Elles constituent l'extension relativiste des fronts d'onde de l'hydrodynamique classique lorsqu' on tient compte des changements de temperature.

Les variétés exceptionnelles $\nabla_{3}^{H}$ peuvent alors généraliser la propagation d'une perturbation faible, compatible au sens d'HUGONIOT avec le mouvement initial du fluide thermodynamique. Elles donnent la solution du problème de la propagation des ondes acoustiques dans le fluide conducteur.

Nous effectuons les calculs dans le système de coordonnées locales où la variété exceptionnelle $\nabla_{3}^{H}$ a pour équation

$$
x^{0}=0 \text {. }
$$

Elle est alors définie par la condition $D=0$, qui s'écrit explicitement :

$$
D=\left|\begin{array}{cccc}
\frac{l}{\rho} u^{0} \frac{\partial \varphi}{\partial p} & x Z & -x g^{00} & x u^{0} \\
u^{0} \frac{\partial \varphi}{\partial p} & \rho+p-x Z & x\left[g^{00}+\left(u^{0}\right)^{2}\right] & -2 x u^{0} \\
g^{00}-\left(u^{0}\right)^{2} & -\left(W^{0}-x Z u^{0}\right) & -x\left[g^{00}-\left(u^{0}\right)^{2}\right] u^{0} & 0 \\
\partial^{0} \theta-Z u^{0} & -x\left(\triangle_{1} \theta-Z^{2}\right) & (\rho+p-x Z)\left(u^{0}\right)^{2} & -V^{0}
\end{array}\right|=0
$$

Ajoutons les éléments de la deuxième ligne multipliés par $u^{0}$ à ceux de la troisième ligne; les éléments de la première ligne à ceux de la seconde ligne, puis les éléments de la quatrième colonne multipliés par $u^{0} \grave{a}$ ceux de la troisième colonne. L'équation précédente devient:

$$
\left|\begin{array}{cccc}
\frac{l}{\rho} u^{0} \frac{\partial \varphi}{\partial p} & x Z & -x\left[g^{00}-\left(u^{0}\right)^{2}\right] & x u^{0} \\
\left(1+\frac{l}{\rho}\right) u^{0} \frac{\partial \rho}{\partial p} & \rho+p & 0 & -x u^{0} \\
g^{00}-\left(u^{0}\right)^{2}+\left(u^{0}\right)^{2} \frac{\partial \varphi}{\partial p} & -2 x\left(\partial^{0} \theta-Z u^{0}\right) & 0 & -2 x\left(u^{0}\right)^{2} \\
\partial^{0} \theta-Z u^{0} & -x\left(\triangle_{1} \theta-Z^{2}\right) & -x\left(\partial^{0} \theta-Z u^{0}\right) u^{0} & -(\rho+p-x Z) u^{0}-x\left(\partial^{0} \theta-Z u^{0}\right)
\end{array}\right|=0 .
$$


Divisons par $V \overline{-\left[g^{00}-\left(u^{0}\right)^{2}\right]}$ les éléments de la première colonne, de la quatrième colonne et ceux de la troisième ligne; puis les éléments de la troisième colonne par $-\left[g^{00}-\left(u^{0}\right)^{2}\right]$. Enfin, multiplions par $x$ les éléments de la quatrième ligne. Il se forme les quantités:

$$
\frac{u^{0}}{\sqrt{-\left[g^{00}-\left(u^{0}\right)^{2}\right]}}=T \quad \frac{x\left(\partial^{00} \theta-Z u^{0}\right)}{\sqrt{-\left[g^{00}-\left(u^{0}\right)^{2}\right]}}=\vec{q} \cdot \vec{n}
$$

où $T$ désigne la vitesse de propagation de la variété $\nabla_{3}^{H}$ et $\vec{n}$ le vecteur unitaire orienté dans l'espace, défini par la composante d'espace de la normale à $\nabla_{3}^{H}$, c' est-à-dire la direction de propagation de l'onde.

Il vient ainsi l'équation:

$$
\left|\begin{array}{cccc}
\frac{l}{\rho} \frac{\partial \varphi}{\partial p} T & x Z & 1 & T \\
\left(1+\frac{l}{\rho}\right) \frac{\partial \varphi}{\partial p} T & \rho+p & 0 & -T \\
-1+\frac{\partial \varphi}{\partial p} T^{2} & 2 \vec{q} \cdot \vec{n} & 0 & -2 T^{2} \\
\vec{q} \cdot \vec{n} & (\vec{q})^{2} & -\vec{q} \cdot \vec{n} T & (\rho+p-x Z) T-\vec{q} \cdot \vec{n}
\end{array}\right|=0
$$

où chaque terme prend aisément sa signification classique.

L'équation (37.2), est du quatrième degré en $T$.

Les racines doivent vérifier la condition $|T| \leq 1$.

On voit que la vitesse de propagation $T$ dépend du champ de température par l'intermédiaire de $\vec{q}$ et $Z$. Or, pour une onde acoustique, où interviennent seulement le gradient de pression et l'accélération $\partial_{0} u^{\lambda}$, les phénomènes thermiques sont négligeables : grad $\theta$ est petit. On peut se rendre compte de l'intérêt de cette étude en faisant une approximation.

Pour cela, nous développons le premier membre de l'équation (37.2) suivant $\partial_{\lambda} \theta$, pris comme infiniment petit principal. En nous limitant aux termes du premier ordre, nous aurons:

$$
\begin{gathered}
-(\rho+p)(\rho+p-x Z) T\left(\frac{\partial \varphi}{\partial p} T^{2}-1\right) \\
+(\rho+p)(\vec{q} \cdot \vec{n})\left\{2\left(1+\frac{l}{\rho}\right) \frac{\partial \varphi}{\partial p} T^{2}-2 T^{2}+\left(\frac{\partial \varphi}{\partial p} T^{2}-1\right)-\frac{2 l}{\rho} \frac{\partial \varphi}{\partial p} T^{4}-\left(\frac{\partial \varphi}{\partial p} T^{2}-1\right) T^{2}\right\}=0 .
\end{gathered}
$$

En simplifiant par $(\rho+p)$ et ordonnant les termes suivant les puissances de $T$, nous avons:

$$
\begin{gathered}
\left.(\vec{q} \cdot \vec{n})\left(1+\frac{\partial l}{\rho}\right) \frac{\partial \varphi}{\partial p} T^{4}+(\rho+p-x Z) \frac{\partial \varphi}{\partial p} T^{3}+\overrightarrow{(q} \cdot \vec{n}\right)\left[1-\left(3+\frac{2 l}{\rho}\right) \frac{\partial \varphi}{\partial p}\right] T^{2} \\
-(\rho+p-x Z) \dot{T}+(\vec{q} \cdot \vec{n})=0
\end{gathered}
$$


Cette équation appelle plusieurs remarques:

a. - Si $\vec{q}=0$, e'est-à-dire si la perturbation satisfait à la loi de détente adiabatique, une racine devient infinie, une deuxième racine tend vers 0 , tandis que les deux autres racines sont données par l'équation:

$$
(p+p-x Z)\left(\frac{\partial \varphi}{\partial p} T^{2}-1\right)=0
$$

Elles ont pour valenr:

$$
T= \pm\left(\frac{\partial \varphi}{\partial p}\right)^{-\frac{1}{2}}
$$

Ces deux racines opposées donnent la vitesse de propagation de l'onde. On retrouve un résultat de l' hydrodynamique classique.

Dans une transformation adiabatique, on peut définir un fluide incom. pressible comme un fluide où les fronts d'onde se propagent avec la vitesse limite unité, ce qui conduit à l'équation d'état:

$$
\rho-p=\text { const. }
$$

b. - $\mathrm{Si} \vec{q}$ est petit, ce qui est le cas des fluides réels ( $x$ est de l'ordre de $10^{-4}$ en unités $C G S$ ), l'équation (37.3) montre qu'une racine est de l'ordre de $\frac{1}{\vec{q} \cdot \vec{n}}$, une racine de l'ordre de $\vec{q} \cdot \vec{n}$, les deux autres racines voisines de $\pm\left(\frac{\partial \varphi}{\partial p}\right)^{-\frac{1}{2}}$, le terme correctif étant de l'ordre de $\vec{q} \cdot \vec{n}$.

Plus exactement :

$$
\begin{array}{ll}
T_{1}=-\frac{1}{1+\frac{2 l}{\rho}} \cdot \frac{\rho+p-x Z}{\vec{q} \cdot \vec{n}} & T_{2}=\frac{\vec{q} \cdot \vec{n}}{\rho+p-x Z} \\
T_{3}=\left(\frac{\partial \varphi}{\partial p}\right)^{-\frac{1}{2}}+\lambda & T_{1}=-\left(\frac{\partial \varphi}{\partial p}\right)^{-\frac{1}{2}}+\lambda^{\prime}
\end{array}
$$

où $\lambda, \lambda^{\prime}$ sont petits.

En substituant dans (37.3), $T= \pm\left(\frac{\partial \varphi}{\partial p}\right)^{-\frac{1}{2}}+\lambda$, nous aurons une equation du 4 è degré en $\lambda$. Mais, puisque $\lambda$ et $\vec{q} \cdot \vec{n}$ sont petits, nous avons une approximation $d u$ ler ordre pour $\lambda$, en nous limitant aux termes du ler ordre en $\lambda$ et en $\overrightarrow{(q} \cdot \vec{n})$

$$
\begin{gathered}
\vec{q} \cdot \vec{n}\left(1+\frac{2 l}{\rho}\right) \frac{1}{\varphi_{p}^{\prime}}+(\rho+p-x Z)\left(\frac{ \pm 1}{\sqrt{\varphi_{p}^{\prime}}}+3 \lambda\right) \\
+\vec{q} \cdot \vec{n}\left[\frac{1}{\varphi_{p}^{\prime}}-\left(3+\frac{2 l}{\rho}\right)\right]-(\rho+p-x Z)\left(\frac{ \pm 1}{\sqrt{\varphi_{p}^{\prime}}}+\lambda\right)+\vec{q} \cdot \vec{n}=0
\end{gathered}
$$


on

$$
(\rho+p) \lambda-\vec{q} \cdot \vec{n}\left(1-\frac{1}{\varphi_{p}^{\prime}}\right)\left(1+\frac{l}{\rho}\right)=0
$$

On en déduit

$$
\lambda=+\left(1-\frac{1}{\varphi_{p}^{\prime}}\right)\left(1+\frac{l}{\rho}\right) \frac{\vec{q} \cdot \vec{n}}{\rho+p}
$$

et on note que $\lambda=\lambda^{\prime}$.

La correction est très petite, puisqu' en unités $C G S$, il faut substituer à $\vec{q}$, le vecteur $\frac{\vec{q}}{c}$, $c$ vitesse limite. Elle est done imperceptible dans les mesures expérimentales.

C'est dans ce sens que nous avons une preuve de la précision des formules classiques proposées depuis LAPLACE.

38. Les variétés exceptionnelles d'ordre zéro $\nabla_{3}^{0}$.

Ce sont des variétés à la traversée desquelles se produisent les discontinuités de $\rho, p, u^{\lambda}, q^{\lambda}$. Nous allons voir $q u^{\prime}$ elles généralisent ce qu'on entend ordinairement par ondes de choc.

Pour les étudier, il nous faut revenir aux équations initiales utilisées pour la détermination de $p, u^{\lambda}$ (cf. $\S 28$ ) que nous prenons sous la forme:

$$
S^{0 \lambda}=\chi T^{0 \lambda} \text {. }
$$

Cette détermination ne fait pas intervenir l'équation de conduction et les conditions de conservation. Nous supposerons donc que les données de

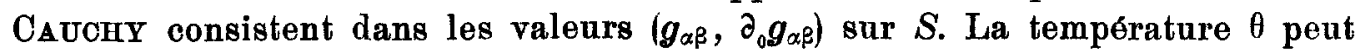
être discontinue à la traversée de $S$. Les conditions de raccordement de l'espace sont préservées.

Nous orientons l'hypersurface $S$ par sa normale $\vec{v}$ de façon à distinguer une face négative et une face positive. Les symboles $\psi_{-}$et $\psi_{+}$représentent les limites en un point $x \in S$, vers lesquelles tend la fonction $\psi\left(x^{\alpha}\right)$ lorsque le point $x^{\alpha}$ varie dans le voisinage correspondant de $S$, pour tendre vers $x$.

Les quantites $S^{0 \lambda}$ ne dépendent que de $g_{\alpha \beta \beta}$ et $\partial_{\lambda} g_{\alpha \beta}$ comme on a vu au $\S 26$. Elles sont donc continues à la traversée de $S$. Des équations (38.1) on déduit :

$$
\left[T^{0 \lambda}\right]=T_{+}^{0 \lambda}-T_{-}^{0 \lambda}=0 .
$$

Le problème consiste à déterminer les valeurs $\rho_{+}, p_{+}, \theta_{+}, u_{+}^{\lambda}, q_{+}^{\lambda}$ lorsqu' on se donne les valeurs $\rho_{-}, p_{-}, \theta_{-}, u_{-}^{\lambda} \cdot q_{-}^{\lambda}$, ou inversement. $C^{\prime}$ est le problème posé dans l'étude des ondes de choc. Les équations (38.2) géné. ralisent donc les équations des ondes de choc.

Nous nous proposons d'écrire les équations en vue des applications. 
Prtam MaU Quan: Sur une théorie relutiviste des fluides, etc.

185

Nous allons prendre dans la suite un espace-temps $\nabla_{4}$ de la relativité restreinte, rapporté à des coordonnés galiléennes réduites. Nous changeons de notation dans les équations (38.1) et (38.2) en remplaçant l'indice 0 par l'indice 1 , réservant ainsi l'indice 0 à la variable temps $x^{0}=c t$.

L'hypersurface $S$ aura alors pour équation locale:

$$
x^{1}=0 \text {. }
$$

Nous choisissons en outre le repère galiléen de façon que l'axe des $x^{1}$ ait la direction $d u$ vecteur vitesse d'espace $\vec{\nabla}$. $\vec{\nabla}$ représente la vitesse des molécules du fluide dans chacun des voisinages de $S$.

Il en résulte que:

$$
u_{0}=\frac{1}{\sqrt{1-\beta^{2}}} \quad u^{i}=\frac{\nabla}{c \sqrt{1-\beta^{2}}} \quad u^{2}=u^{3}=0 \quad\left(\beta=\frac{\nabla}{c}\right) .
$$

L' hypersurface $S$ doit être orienté dans le temps, $g^{11}=-1$. Les equations

$$
T_{+}^{1 \lambda}=T_{-}^{1 \lambda}
$$

s'écrivent explicitement :

$$
\begin{aligned}
\left(\rho_{+}+p_{+}\right) u_{+}^{1} u_{+}^{0}-\left(u_{+}^{0} q_{+}^{1}+u_{+}^{1} q_{+}^{0}\right) & =\left(\rho_{-}+p_{-}\right) u_{-}^{1} u_{-}^{0}-\left(u_{-}^{0} q_{-}^{1}+u_{-}^{1} q_{-}^{0}\right) \\
(38.5)\left(\rho_{+}+p_{+}\right)\left(u_{+}^{1}\right)^{2}+p_{+}-\left(u_{+}^{1} q_{+}^{1}+u_{+}^{1} q_{+}^{1}\right) & =\left(\rho_{-}+p_{-}\right)\left(u_{-}^{1}\right)^{2}+p_{-}-\left(u_{-}^{1} q_{-}^{1}+u_{-}^{1} q_{-}^{1}\right) \\
-u_{+}^{1} q_{+}^{2} & =-u_{-}^{1} q_{-}^{2} \\
-u_{+}^{1} q_{+}^{3} & =-u_{-}^{1} q_{-}^{3} .
\end{aligned}
$$

A ces équations il faut adjoindre les hypothèses (\$ 22)

$$
\begin{aligned}
& \rho=\mu(1+\varepsilon) \\
& \nabla_{\alpha}\left(\mu u^{\alpha}\right)=0 .
\end{aligned}
$$

A partir de l'équation (38.7), il est possible de déduire une équation analogue à une relation connue dans l'étude des ondes de choc.

Choisissons en effet un domaine $B$ limité par deux surfaces voisines et parallèles à $S$ :

$$
x^{1}=-\varepsilon \quad x^{1}=+\varepsilon
$$

dans un tube de courant. Intégrons l'équation (38.7) sur ce domaine :

$$
\iiint \int_{B} \nabla_{\alpha}\left(\mu u^{\alpha}\right) d \tau=\iiint_{\partial B} \mu u^{\alpha} \nu_{\alpha} d \omega=0
$$

dw est l'élément d'aire de la frontière $\partial B$ de $B$. En vertu du choix du domaine $B$, on a

$$
\iiint_{S_{+\varepsilon}} \mu u^{\alpha{ }_{x}} d \omega-\iiint_{S_{-\varepsilon}} \mu u^{\alpha} v_{x} d \omega=0
$$


$v_{x}$ désigne les composantes de la normale aux hypersurfaces $x^{1}=$ const. Ici, on a $v_{0}=v_{2}=v_{3}=0, v_{1}=1$. En faisant tendre $\varepsilon$ vers 0 , on déduit de l'égalité précédente, l'équation cherchée:

$$
\mu_{+} u_{+}^{1}=\mu_{-} u_{-}^{1} .
$$

Les équations (38.5) (38.6) (38.7) et (38.8) constituent l'extension relativiste des équations d'Hugoniot pour une onde de choc, compte tenu des phénomènes thermiques. Il convient de leur adjoindre l'équation d'état.

Il est facile de mettre les equations dans le cas adiabatique $\overrightarrow{(q}=0)$ sous la forme:

$$
\begin{gathered}
\frac{\mu_{+} V_{+}}{c \sqrt{1-\beta_{+}^{2}}}=\frac{\mu_{-} \nabla_{-}}{c \sqrt{1-\beta_{-}^{2}}}=m \\
\frac{m\left(1+\varepsilon_{+}+\frac{p_{+}}{\mu_{+}}\right)}{\sqrt{1-\beta_{+}^{2}}}=\frac{m\left(1+\varepsilon_{-}+\frac{p_{-}}{\mu_{-}}\right)}{\sqrt{1-\beta_{-}^{2}}} \\
\frac{m\left(1+\varepsilon_{+}+\frac{p_{+}}{\mu_{+}}\right) \nabla_{+}}{c \sqrt{1-\beta_{+}^{2}}}+p_{+}=\frac{m\left(1+\varepsilon_{-}+\frac{p_{-}}{\mu_{-}}\right) V_{-}}{c \sqrt{1-\frac{\beta_{-}^{2}}{2}}+p_{-}}
\end{gathered}
$$

On en tire les corrections relativistes vis-à-vis des équations de Hugoniot. 
CHAPITRE V.

MOUVEMENTS PERMANENTS ET APPLICATIONS

A DES MODELES D' UNIVERS THERMODYNAMIQUES STATIONNAIRES

\section{I. - Mouvements permanents d'un fluide parfait thermodynamique.}

39. Espace-temps stationnaire dans un domaine.

L'étude des monvements permanents d'un schéma thermodynamique est liée à la théorie des espaces-temps stationnaires. Il est indispensable de rappeler quelques définitions générales.

La métrique de l'espace-temps $\nabla_{4}$ est dite localement stationnaire dans un domaine d'un système de coordonnées locales $\left(x^{0}, x^{1}\right)$, si celles-ci peuvent être choisies de façon que les potentiels correspondants $g_{\alpha \beta}$ soient indépendants de la variable temps $x^{0}$, les lignes le long desquelles cette variable $x^{0}$ varie seule, étant orientées dans le temps.

L'espace-temps est dit stationnaire dans un domaine $D_{4}$, si la varieté riemannienne définie par $D_{4}$ munie de la métrique d'univers $d s^{2}=g_{x \beta} d x^{\alpha} d x^{\beta}$, admet un groupe connexe $d$ 'isométries globales à un paramètre, ne laissant invariant aucun point de $D_{4}$ et dont les trajectoires $z$ sont orientées dans le temps.

On suppose de plus que les $z$ sont homéomorphes à la droite réelle $R$ et qu'on pent trouver une variété différentiable a trois dimensions $\nabla_{s}$, satisfaisant aux mêmes hypothèses de différentiabilité que $\nabla_{4}$, telle qu'il existe un homéomorphisme différentiable de classe $C^{2}$, de $D_{4}$ sur le produit topologique $\nabla_{3} \times R$.

On sait alors que le vecteur $\vec{\xi}$ générateur infinitésimal du groupe d'isométries, satisfait aux équations de KILLING

$$
X g_{x \beta} \equiv \nabla_{x} \xi_{\beta}+\nabla_{\beta} \xi_{\alpha}=0
$$

où $X$ désigne l'opérateur de dérivation de LIE relatif au vecteur $\vec{\xi}$.

De l'homéomorphisme de $D_{4}$ sur $\nabla_{3} \times R$, il est possible de déduire des systèmes de coordonnées $\left(x^{0}, x^{4}\right)$ dits adaptés au caractère stationnaire de $\nabla_{\text {a }}$ tels que les lignes de temps, trajectoires du champ de vectcurs $\vec{\xi}$, soient les lignes $x^{i}=$ const., et que la composante $\xi^{0}$ de $\vec{\xi}$ soit égale à 1 .

Dans ce système de coordonnées

$$
\begin{gathered}
\xi_{\alpha}=g_{0 x} \\
X g_{\alpha \beta} \equiv \nabla_{\alpha} \xi_{\beta}+\nabla_{\beta} \xi_{\alpha}=\partial_{0} g_{\alpha \beta}=0 .
\end{gathered}
$$


Les $g_{\alpha \beta}$ sont indépendants de la variable $x^{0}$. La métrique de $V_{4}$ est localement stationnaire au voisinage de tout point de $D_{4}$.

Dans ce même système de coordonnées, l'opérateur de dérivation de LrE, appliqué à une fonction scalaire $F$ ou à un vecteur $\vec{u}$ se réduit à :

$$
\begin{gathered}
X F \equiv \xi^{\alpha} \partial_{\alpha} F=\partial_{0} F \\
X u_{\beta} \equiv \xi^{\alpha} \nabla_{\alpha} u_{\beta}+u_{\alpha} \nabla_{\beta} \xi^{\alpha}=\nabla_{0} u_{\beta}+\Gamma_{0 \beta}^{x} u_{\alpha}=\partial_{\jmath} u_{\beta} .
\end{gathered}
$$

40. Définition des mouvements permanents.

Etant donné un milieu thermodynamique en mouvement, associé à une métrique d'univers $d s^{2}$ dans un domaine $D_{4}$ de l'espace-temps $V_{4}$, nous dirons que le mouvement de ce milieu est permanent si les hypothèses suivantes sont satisfaites:

a. - l'espace-temps riemannien $\nabla_{4}$ est stationnaire dans le domaine $D_{4}$ :

b. - le groupe d'isométries laisse invariants le vecteur vitesse unitaire $\vec{u}$. le tenseur des pressions $\pi_{\alpha \beta}$ et la température $\theta$ du milieu.

On a ainsi les équations

$$
X \vec{u}=0 \quad X \pi_{\times \beta}=0 \quad X \theta=0 .
$$

Si les coordonnées locales choisies dans le domaine $D_{4}$ sont adaptées au caractère stationnaire de l'espace-temps $V_{4}$, les conditions précédentes se réduisent à

$$
\partial_{0} u_{\lambda}=0 \quad \partial_{0} \pi_{\alpha \beta}=0 \quad \partial_{0} \theta=0 .
$$

Les composantes $u_{\lambda}$ du vecteur-vitesse unitaire sont constantes le long des lignes de temps, ainsi que celles du tenseur des pressions et que la température.

Dans le cas d'un fluide parfait thermodynamique, la deuxième équation se réduit à $X p=0$, et, en coordonnées adaptées à

$$
\partial_{0} p=0 \text {. }
$$

41. Etude des mouvements permanents du fluide parfait thermodynamique. Soit un schéma fluide parfait thermodynamique à équation d'etat

$$
\rho=\varphi(p, \theta)
$$

et tel que l'espace-temps $\nabla_{4}$ associé au domaine $D_{4}$ occupé par ce fluide, soit stationnaire dans le domaine $D_{4}$.

Dans la suite, nous supposerons choisi un système de coordonnées adapté $\left(x^{i}, x^{i}\right)$.

Considérons un mouvement de ce fluide.

Soit $x$ un point de $D_{4}$ appartenant à la variété $S$ d'équation $x^{0}=0$, supposée non exceptionnelle pour le problème de CAUCHY relatif an fluide considéré. 
Pham MaU Quan: Sur une théorie relatiriste des fluides, etc.

Sur la variété $S$ et sur les variétés voisines d'équation $x^{\circ}=$ const., nous avons les trois égalités suivantes (cf. $§ 28$; éq. (28.6) (28.7) et (28.8)) :

$$
\begin{gathered}
\chi\left(\nabla^{0}+x 2^{00} \theta\right) u^{0}=S^{00}+\chi p g^{00} \\
\chi\left(\nabla^{0} Z+\chi \triangle_{1} \theta u^{0}\right)=\left(S^{0 \lambda}+\chi p g^{0 \lambda}\right) \partial_{\lambda} \theta \\
\chi^{2}\left[\left(\nabla^{0}\right)^{2}+2 x \nabla^{0} Z u^{0}+x^{2} \triangle_{1} \theta\left(u^{0}\right)^{2}=g^{\lambda \mu}\left(S_{\lambda}^{0}+\chi p g_{\lambda}^{0}\right)\left(S_{\mu}^{0}+\chi p g_{\mu}^{0}\right)\right.
\end{gathered}
$$

où

$$
V^{0}=(p+p-x Z) u^{0}+x\left(\partial^{0} \theta-Z u^{0}\right)
$$

$Z=u \partial_{\rho} \theta$ et où le vecteur courant de chaleur $q^{\alpha}$ est défini par

$$
\boldsymbol{\theta}^{\alpha}=-x\left(\partial^{\alpha} \theta-Z u^{\alpha}\right)
$$

Nous ferons l'hypothèse suivante :

$$
\text { espace-temps stationnaire }
$$

$$
\partial_{0} \theta=0
$$

$$
\partial_{0} q^{0}=c \rho u^{0} \partial_{0} \theta-\frac{l}{\rho} u^{0} \partial_{0} \rho
$$

en coordonnées adaptées. Mais nous conservons la dérivée $\partial_{00} \theta$ dans les calculs. Il en résulte que:

$$
\begin{gathered}
\partial_{0}\left(\partial^{0} \theta\right)=\partial_{0}\left(g^{0 \lambda} \partial_{\lambda} \theta\right)=g^{00} \partial_{00} \theta \\
\partial_{0} \triangle_{1} \theta=2 \partial \partial^{0} \theta \partial_{00} \theta .
\end{gathered}
$$

Nous allons étudier la variation des différentes quantités introduites dans les équations, par les transformations du groupe d'isométries. Pour cela, il nous suffit de suivre leur variation le long des trajectoires d' isométrie. Nous dérivons done les equations (41.1) (41.2) (41.3) par rapport à $x^{0}$, en tenant compte de l'equation d'état, et nous explicitons l'hypothèse (41.4).

En coordonnées adaptées, on a :

$$
\partial_{0} g_{\alpha \beta}=0 \quad \partial_{0} S^{0 \lambda}=0 .
$$

La dérivation annoncée conduit aux équations suivantes où l'on a posé:

$$
\begin{gathered}
W^{0}=\nabla^{0}+x \partial^{0} \theta \\
g^{00} \partial_{0} p-W^{0} \partial_{0} u^{0}-x u^{0} g^{00} \partial_{00} \theta-u^{0} \partial_{0} V^{0}=0 \\
\partial^{0} \theta \partial_{0} p-x \triangle_{1} \theta \partial_{0} u^{0}+\left(W^{0}-2 x \partial^{0} \theta\right) u^{0} \partial_{00} \theta-V^{0} \partial_{0} Z-Z \partial_{0} V^{0}=0 \\
W^{0} u^{0} \partial_{0} p-x\left(Z V^{0}+x \triangle_{1} \theta u^{0}\right) \partial_{0} u^{0} j+x^{2}\left(u^{0}\right)^{\circ} \partial^{0} \theta \partial_{00} \theta \\
+-x V^{0} u^{0} \partial_{0} Z+\left(V^{0}+x Z u^{0}\right) \partial_{0} \nabla^{0}=0 \\
\frac{l}{\rho} u^{0} \frac{\partial \varphi}{\partial p} \partial_{0} p+x Z \partial_{0} u^{0}-x g^{00} \partial_{00} \theta+x u_{0} \partial^{0} Z=0 .
\end{gathered}
$$


A ces quatre équations, il nous faut ajouter la relation suivante tirée de l'expression de $V^{0}$, par dérivation

$$
\left(\frac{\partial \varphi}{\partial p}+1\right) u_{0} \partial^{0} p+(\rho+p-2 x Z) \partial_{0} u^{0}+x g^{00} \partial_{00} \theta-\partial_{0} V^{0}=0 .
$$

En multipliant l'équation $\left(41.2^{\prime}\right)$ par $x u^{\circ}$, puis l'ajoutant à $\left(41.3^{\prime}\right)$, il vient:

$$
\nabla^{0}\left[u^{0} \partial_{0} p-x Z \partial_{0} u^{\mathfrak{c}}-x\left(u^{0}\right)^{2} \partial_{00}{ }^{\theta}-\partial_{0} V^{0}\right]=0 .
$$

La variêté $S\left(x^{0}=0\right)$ étant supposée non exceptionnelle pour le problème de CaUOHY, $V^{0} \neq 0$ (cf. $\S 30$ ), on en déduit:

$$
\partial_{0} V^{0}=\left[u_{0} \partial_{0} p-x Z \partial_{0} u^{0}-x\left(u^{0}\right)^{2} \partial_{00} \theta\right. \text {. }
$$

En portant cette valeur de $\partial_{0} \nabla^{0}$ dans les equations (41.1') (41.2') (41.5), en conservant l'équation $\left(41.4^{\prime}\right)$ nous obtenons finalement le système:

$$
\begin{gathered}
{ }_{\rho}^{l} u^{0} \frac{\partial \varphi}{\partial p} \partial_{0} p+x Z \partial_{0} u^{0}-x g^{00} \partial_{00} \theta+x u_{0} \partial^{0} Z=0 \\
u^{0} \frac{\partial \varphi}{\partial p} \partial_{0} p+\left(\rho+p-x Z \partial_{0} u^{0}+x\left[g^{00}+\left(u^{0}\right)^{2}\right] \partial_{00} \theta-2 x u_{0} \partial^{0} Z=0\right. \\
{\left[g^{00}-\left(u^{0}\right)^{2}\right] \partial_{0} p-\left(W^{0}-x Z u^{0}\right) \partial_{0} u^{0}-x u^{0}\left[g^{00}-\left(u^{0}\right)^{2}\right] \partial_{00} \theta=0} \\
\left(\partial^{0} \theta-Z u^{0}\right) \partial_{0} p-x\left(\triangle_{1} \theta-Z^{2}\right) \partial_{0} u^{0}+(\rho+p-x Z)\left(u^{0}\right)^{2} \partial_{00} \theta-V^{0} \partial_{0} Z=0
\end{gathered}
$$

linéaire et homogène par rapport aux inconnues.

Le déterminant de ce système est identique au déterminant $D$ auquel on est conduit dans l'étude du problème de CAUCHY au chapitre IV. Or, la variété $S$ est supposée non exceptionnelle. $D$ n'est pas nul. Le système des équations précédentes admet done la solution unique:

$$
\partial_{0} p=\partial_{0} u^{0}=\partial_{00} \theta=\partial_{0} Z=0 .
$$

On en déduit également $\partial_{0} V^{0}=\partial_{0} \rho=0$. En se reportant à l'expression de $u_{\lambda}$, calculée dans l'étude du problème de OAUCHY (§ 28), ainsi qu'à la définition $d u$ vecteur courant de chaleur, on en déduit également

$$
\partial_{0} u^{\lambda}=0 \quad \partial_{0} q^{\lambda}=0 .
$$

Il en est de même de toute caractéristique du fluide définie à partir des quantités $\rho, p, \theta, u^{\lambda}$. Elle reste constante le long des trajectoires d'isométries.

Nous pouvons énoncer le théorème.

THEORÈv. - Étant donné dans un domaine $D_{4}$ de l'espace-temps, un fuide parfait thermodynamique admettant une équation d'élat, pour que le mouvement de ce fuide soit permanent, il faut et il suffit que maine $D_{4}$.

a. - l'espace-temps riemannien associé soit stationnaire dans le do. 
Phan MaU Quan: Sur mue thérie relativiste des fluides, etc.

b. - le groupe d'isométries laisse invariante la température $\theta$ et qu'en coordonnées adaptées, la composante contravariante $q^{0}=-x\left(\partial^{\circ} \theta-Z u^{\circ}\right) d u$ vecteur courant de chaleur, dans la direction des trajectoires d'isométries. satisfasse à la relation

$$
\partial_{\bullet} q^{0}=c \rho u^{0} \partial_{0} \theta-\frac{l}{\rho} u^{0} \partial_{0} \rho \text {. }
$$

S'il en est ainsi, le groupe d'isométries laisse invariants la pression $p$, le vecteur vitesse unitaire $u^{\lambda}$, le vecteur courant de chaleur $q^{\lambda}$, ainsi que toute caractéristique définie à partir des quantités $\rho, p, u^{\lambda}, \theta$.

\section{II. - Notion globale d'espace-temps stationnaire.}

42. Definition globale d'espace-temps stationnaire.

Nous terminerons notre étude mathématique de la théorie relativiste des fluides thermodynamiques, par l'application à des modeles d'univers thermodynamiques stationnaires. Nous allons rappeler d'abord les résultats essentiels de la théorie globale des espaces-temps stationnaires. On trouvera un exposé de cette théorie, dans l'ourrage de M. Lichnerowicz consacré à l'étude mathématique des théories relativistes de la gravitation et de l'électromagnétisme (voir bibliographie).

Les notions qui vont être rappelées, sont indispensables à la compré. hension de notre dernière étude. Nous exprimons toute notre reconnaissance à M. LICHNERowicz de nous avoir permis d'en faire usage.

Un espace-temps riemannien $V_{4}$ est dit stationnaire, s'il est stationnaire dans toute' sa variété, c' est-à-dire s'il existe un groupe connexe à un paramètre d'isométries globales de $V_{4}$, à trajectoires $z$ orientées dans le temps, ne laissant invariant aucun point de $\nabla_{4}$. La famille des lignes $z$ appelées lignes de temps, doit satisfaire aux hypotheses suivantes:

a. - Les $Z$ sont homéomorphes à la droite réelle $R$.

b. - on peut trouver une variété différentiable à trois dimensions $V_{3}$, satisfaisant aux mêmes hypothèses de différentiabilité que $V_{4}$, tel qu'il existe un homéomorphisme différentiable de classe $C^{2}$ de la variété $V_{4}$ sur le produit topologique $V_{3} \times R$, dans lequel les $z$ s'appliquent sur les droites facteurs. Cet homéormorphisme est de plus supposé continu par morceaux jusqu'à l'órdre 4.

On peut identifier $V_{3} \grave{a}$ l'espace dont les points $z$ sont lignes de temps de $V_{4} . \nabla_{3}$ sera dit espace-quotient, ou plus brièvement, espace.

Il existe alors dans. $\nabla_{4}$ des coordonnées locales adaptées au caractère stationnaire de $V_{4}$, jouissant des propriétés suivantes:

1. - les $\left(x^{i}\right)$ sont un système de coordonnées locales arbitraires de $\nabla_{3}$. Les variétés $x^{0}=$ const. sont des variétés globalement définies dans $V_{4}$ et homéomorphes à $\nabla_{3}$. Elles seront appelées sections d'espace $W_{3}$. 
2. - les potentiels relatifs aux coordonnées adaptées $\left(x^{0}, x^{4}\right)$ sont indépendants de la variable $x^{0}$.

3. - le vectenr $\vec{\xi}$ générateur infinitésimal du groupe d'isométries, admet pour composantes contravariantes $\xi^{i}=0, \xi^{0}=1$, le carré de ce vecteur est

$$
\xi^{2}=g_{00}>0 \quad\left(\xi=\sqrt{\xi^{\overline{2}}}>0\right) .
$$

4. - ces coordonnées sont définies à un changement de variables près

$$
\begin{gathered}
x^{0^{\prime}}=x^{0}+\psi\left(x^{k}\right) \\
x^{u}=\psi^{\prime \prime}\left(x^{k}\right)
\end{gathered}
$$

où $\psi$ désigne la restriction à une carte locale de $\nabla_{3}$ d'une fonction $\psi(z)$ definie sur $V_{3}$.

Dans la suite, nous n'introduirons que des systèmes de coordonnées locales adaptées.

43. Les espaces riemanniens $V_{3}$ et $W_{3}$.

Sur chaque section d'espace $W_{3}$, la métrique de $\nabla_{4}$ avec son groupe d'isométries définit des tenseurs. Ainsi les $g_{i j}$ définissent sur $W_{3}$ un tenseur symétrique, les $g_{0 i}=\xi_{i}$ un vecteur covariant, $g_{00}=\xi^{2}$. un scalaire, parce que ces quantités se transforment tensoriellement dans le changement de coordonnées locales

$$
\begin{gathered}
x^{0 \prime}=x^{0} \\
x^{\prime \prime}=\psi^{\prime \prime}\left(x^{k}\right)
\end{gathered}
$$

qui conserve $W_{3}$.

Parmi ces tenseurs, certains tels que $g_{00}=\xi^{2}$ viennent s'appliquer les uns sur les autres, dans l'application induite par l'homéomorphisme de deux sections d'espace de systèmes différents. Ils peuvent ainsi être considérés comme définis sur l'espace quotient $V_{3}$. Ils doivent rester invariants par le changement de coordonnees

$$
\begin{gathered}
x^{0^{\prime}}=x^{0}+\psi\left(x^{k}\right) \\
x^{\iota^{\prime}}=x^{i}
\end{gathered}
$$

qu' on appelle un changement de systèmes de sections d'espace.

A chaque point $x$ d'un voisinage de $\nabla_{4}$, on peut associer un repère orthonormé dont le premier vecteur $\overrightarrow{e_{0}}$ soit le vecteur unitaire tangent en $x$ a la ligne de temps $z(x)$ passant par $x$. Il lui correspond alors un système de formes de PFAFF locales $\omega_{\text {x }}$.

$$
\overrightarrow{d \vec{x}}=\omega^{0} \overrightarrow{e_{0}}+\omega_{-}^{i} \overrightarrow{e_{i}} .
$$

La métrique de $\nabla_{4}$ prend la forme:

$$
d s^{2}=\left(\omega_{-}^{0}\right)^{2}-\sum_{i=1}^{3}\left(\omega_{-}^{i}\right)^{2} .
$$


Pham MaU Quan: Sur une théorie relativiste des fluides, etc.

193

Un tel repère orthonormé sera dit adapté, et sera distingué par des indices soulignés.

La formule (43.4) n'est autre qu'une décomposition en carrés de la forme quadratique fondamentale attachée à $\nabla_{4}$, la variable $x^{0}$ y joue le rôle de variable directrice. On en deduit :

$$
\begin{gathered}
\omega_{-}^{0}=\frac{1}{\xi}\left(g_{00} d x^{0}+g_{0 i} d x^{i}\right) \\
-\sum_{i=1}^{3}\left(\omega_{-}^{i}\right)^{2}=\left(g_{i j}-\frac{g_{0 i} g_{0 j}}{g_{00}}\right) d x^{i} d x^{j} .
\end{gathered}
$$

La forme quadratique $\left.d^{*} s^{2}=-\sum_{i=1}^{3}(\omega)^{i}\right)^{2}$ est independante de la variable $x^{\theta}$ et indépendante de tout système de sections d'espace. Elle définit sur $V_{3}$ une métrique définie négative. Nous poserons

$$
\stackrel{*}{g}_{i j}=g_{i j}-\frac{g_{0 i} g_{0 j}}{g_{00}} \text {. }
$$

Le tenseur conjugué a pour valeur:

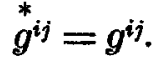

Nous supposerons dans la suite que les variétés $V_{3}$ et $W_{3}$ sont donées de la structure de variété riemannienne

$$
d s^{*}=\stackrel{*}{g}_{i j} d x^{i} d x^{j}
$$

44. Rappel des formules fondamentales dans $V_{3}$ et $W_{4}$.

Si on introduit le vecteur $\varphi_{\lambda}$ défini dans $V_{4}$ par

$$
\varphi_{\lambda}=\frac{\xi_{\lambda}}{\xi^{2}}=\frac{g_{0 \lambda}}{g_{00}} \quad\left(\varphi_{0}=1\right)
$$

on vérifie que les composantes $H_{0 \lambda}=\partial_{0} \varphi_{\lambda}-\partial_{\lambda} \varphi_{0}$ de son rotationnel $H_{\lambda \mu}$ sont nulles. Les $H_{i j}$ définissent ainsi un tenseur sur $V_{3}$.

Le tenseur d'espace $H_{i j}$ a une signification géométrique importante. Sa nullité entraîne que les lignes de temps sont trajectoires orthogonales des sections d'espace locales.

Dans l'espace-quotient $V_{3}$, on a ainsi défini un scalaire $\xi$, une métrique riemannienne définie négative $\stackrel{*}{*} s^{z}$ et un tenseur antisymétrique $H_{i j}$. Sur chaque section d'espace $W_{3}$, se trouve défini outre ces éléments communs à $V_{3}$, un champ de vecteurs $\varphi_{i}$ tel que

$$
\begin{aligned}
& \stackrel{*}{g}_{i j}=g_{i j}-\xi^{2} \varphi_{i} \varphi_{j} \\
& H_{i j}=\partial_{i} \varphi_{j}-\partial_{j} \varphi_{i} .
\end{aligned}
$$


On pent évaluer les composantes du tenseur de RIoci de $\nabla_{4}$ en repère orthonormé adapté, en fonction de celles de $\nabla_{3}$ et des différents tenseurs introduits. Voici les relations établies par M. LICHNERowiCz:

$$
\begin{aligned}
& R_{\underline{i} \underline{k}}=\stackrel{*}{R}_{\underline{i} \underline{k}}-\frac{1}{\xi} \stackrel{*}{\nabla}_{\underline{k}}\left(\partial_{\underline{i}} \xi\right)-\frac{\xi^{2}}{2} H_{\underline{i}} \underline{j} H_{\underline{k} \underline{j}} \\
& R_{i \underline{0}}=\frac{1}{2 \xi^{2}} \stackrel{*}{\nabla}_{\underline{\underline{j}}\left(\xi^{3} H_{\underline{i}}\right)} \\
& R_{\underline{0} \underline{0}}=-\frac{1}{\xi} \stackrel{*}{\nabla} \xi+\frac{\xi^{2}}{2} H^{2}
\end{aligned}
$$

où

$$
H^{2}=\frac{1}{2} H_{\underline{i} \underline{j}} H^{i \underline{j}}
$$

l'astérique * indique que l'opérateur correspondant est relatif à la métrique $d s^{*}$ de $\nabla_{3}$.

Dans une section d'espace $W_{3}$, la composante $R_{0}{ }^{0}$ du tenseur de RIcor présente le caractère scalaire. On peut la calculer dans le repère naturel associé an système de coordonnées locales adaptées.

En effet, si $\left(\vec{e}_{\alpha}\right)$ est le repère naturel en $x$, on $a$ :

$$
d \vec{x}=d x^{\alpha} \overrightarrow{e_{\alpha}}=\omega \underline{\underline{a}} \overrightarrow{\underline{e_{\alpha}}} .
$$

Soit $\left(A_{\beta} \underline{a}\right)$ la matrice de passage des repères, et $\left(A_{\underline{\beta}}^{\alpha}\right)$ la matrice inverse. A partir des formules (43.4) et (44.1), on établit les valeurs suivantes

$$
\begin{array}{lll}
A_{0}{ }^{0}=\xi & A_{i}{ }^{0}=\xi \varphi_{\underline{i}} & A_{0} \underline{i}=0 \\
A_{\underline{0}}^{0}=\frac{1}{\xi} & A_{\underline{i}}^{0}=-\varphi_{\underline{i}} & A_{\underline{0}}^{i}=0 .
\end{array}
$$

Il en résulte que

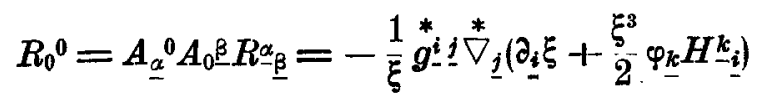

et on est conduit à introduire le vecteur $\vec{h}$ de $W_{3}$ defini par ses composantes en repère orthonormé

$$
h_{i}=\partial_{\underline{i}} \xi+\frac{\xi^{3}}{2} \varphi_{\underline{k}} H_{-\underline{i}}^{k_{i}} .
$$


En vertu du caractère tensoriel sur $W_{3}$ des différents termes introduits, on peut faire intervenir des composantes relatives aux coordonnées locales, et ecrire la formule:

$$
h_{i}=\hat{c}_{i} \xi+\frac{\xi^{3}}{2} \varphi_{k} H_{i}^{k_{i}}
$$

et

$$
R_{0}^{0}=-\frac{1}{\xi} \operatorname{div}^{*} \vec{h}
$$

Si nous introduisons le vecteur $\vec{p}$ de composantes

$$
p_{i}=\partial_{i} \xi-h_{i}=\frac{\xi^{3}}{2} \varphi_{k} H_{i}^{k}
$$

la formule (44.7) pent s'écrire

$$
R_{0}^{0}=-\frac{1}{\xi} \stackrel{*}{\Delta \xi}+\frac{1}{\xi} \operatorname{div}^{*} \vec{p} .
$$

D' autre part, d'après (44.5), $R_{0 !}=A_{0} \underline{\alpha} A_{0} \underline{\beta} R_{\alpha \underline{\beta}}=\xi^{2} R_{00}$. La troisième relation (44.3) fournit alors:

$$
\frac{R_{00}}{g_{00}}=-\frac{1}{\xi} \stackrel{*}{\xi} \xi+\frac{\xi^{2}}{2} H^{2}
$$

soit, en retranchant membre à membre avec (44.8)

$$
\xi\left|\frac{R_{00}}{g_{00}}-R_{0}^{0}\right|=\frac{\xi^{3}}{2} H^{2}-\operatorname{div} \vec{p} .
$$

Or, on a manifestement $R_{00}=g_{00} R_{0}{ }^{0}+g_{0 i} R_{0}{ }^{i}$. En remarquant que $\frac{g_{0 i}}{g_{00}}=\varphi_{i}$, on en déduit

$$
\xi_{\rho_{i} R_{0}}{ }=\frac{\xi^{3}}{2} H^{2}-\operatorname{div}^{*} \vec{p}
$$

Soit $C$ une chaîne différentiable de dimension 3 dans $W_{3}$, de bord $a C$. Par intégration sur $C$, on obtient la formule intégrále

$$
\iiint_{C} \xi \varphi_{i} R_{0}{ }^{i} d \tau=\iiint_{C}^{\xi^{3}} \frac{{ }^{3}}{2} H^{2} d \tau-\operatorname{flux}_{\partial C}^{*} \vec{p}
$$

où $d \tau$ désigne l'élément de volume de $W_{3}$ munie de la métrique $d s^{*}$. 
45. Conduction de chaleur en régime permanent.

Si le mouvement d'un milieu fluide parfait thermodynamique est permanent, la température $\theta$ ne dépend pas de la variable $x^{\circ}$. Il en résulte que $\theta\left(x^{i}\right)$ est une fonctión scalaire de classe $C^{2}$ définie sur $W_{3}$. Son gradient $y$ définit un champ de vecteurs covariants.

D'autre part, le changement de coordonnées (43.1) qui conserve $W_{3}$, transforme les composantes du vecteur vitesse unitaire $u^{\lambda}$, suivant les formules

$$
u^{0^{\prime}}=u^{0} \quad u^{\prime \prime}=u^{k} \frac{\partial \psi^{\prime \prime}}{\partial x^{k}} .
$$

On peut regarder les $u^{\prime}$ comme les composantes d'un vecteur contravariant défini sur $W_{3}$.

Nous pouvons faire des remarques analogues sur le vecteur courant de chaleur

$$
\begin{aligned}
& q^{i}=-x\left(\partial^{2} \theta-Z u^{0}\right) \\
& q^{0}=-x\left(\partial^{\circ} \theta-Z u^{0}\right)
\end{aligned}
$$

$Z=u_{p}^{p} \partial_{\rho} \theta$ est un scalaire défini dans $W_{3}$; il en résulte que $q^{i}$ sont les com. posantes d'un vecteur contravariant défini sur $W_{3}$. On notera le caractère scalaire sur $W_{3}$ de $u^{0}, \partial^{0} \theta, q^{0}$.

L'équation de conduction de la chaleur s'écrit

$$
\nabla_{\alpha} q^{\alpha} \equiv \partial_{x} q^{\alpha}+\Gamma_{\alpha}^{\alpha} q^{\beta}=c \rho Z-\frac{l}{\rho} u^{\alpha} \partial_{\alpha} \rho .
$$

Dans le système de coordonnées adaptées choisi, $\partial_{0} q^{0}=0$ et $\Gamma_{\alpha}{ }_{0}=$ $=\partial_{0} \log \sqrt{|g|}=0, \partial_{0} \rho=0$, de sorte que l'équation (45.1) se réduit à

$$
\partial_{i} q^{i}+\Gamma_{a}{ }^{\alpha} q^{j}=c \rho Z-\frac{l}{\rho} u^{i} \partial_{i} \rho .
$$

Le second membre de cette équation a le caractère scalaire sur $W_{3}$. Nous nous proposons de transformer l'équation de conduction dans $\nabla_{4}$, en une formule de divergence dans $W_{3}$.

Pour cela, cherchons à évaluer la quantité ${\stackrel{*}{\Gamma_{i}}}_{i}{ }_{j}$ dans la métrique $d^{*} s^{*}$ de $W_{3}$. Nous avons:

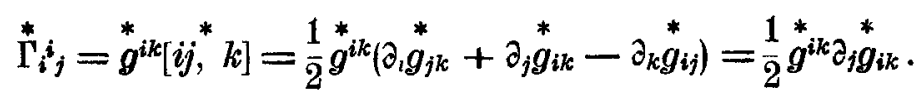

Substituons aux $g_{i k}$ leurs valeurs en fonction des $g_{\alpha \beta}$ :

$$
\begin{gathered}
\stackrel{*}{\Gamma_{i}{ }_{j}}=\frac{1}{2} g^{i k}\left\{\partial_{j} g_{i k}-\partial_{j}\left(\frac{g_{0 i} g_{0 k}}{g_{00}}\right)\right\} \\
=\frac{1}{2} g^{i k}\left\{\partial_{j} g_{i k}-\frac{1}{\left(g_{00)^{2}}\right.}\left(g_{00} g_{0 i} \partial_{j} g_{0 k}+g_{00} g_{0 k} \partial_{j} g_{0 i}-g_{0 i} g_{0 k} \partial_{j} g_{00}\right)\right\} .
\end{gathered}
$$


En remarquant que :

$$
\begin{gathered}
g^{i k} g_{0 i}=g^{\alpha k} g_{0 x}-g^{0 k} g_{00}=-g_{00} g^{0 k} \\
g^{i k} g_{0 i} g_{0 k}=-g_{00} g^{0 k} g_{0 k}=-g_{00}\left(1-g^{00} g_{00}\right)
\end{gathered}
$$

nous aurons :

$$
\stackrel{*}{\Gamma_{i}^{i} j}=\frac{1}{2}\left[g^{i k} \partial_{j} g_{i k}+g^{i 0} \partial_{j} g_{i 0}+g^{0 k} \partial_{j} g_{0 k}+g^{00} \partial_{j} g_{00}\right]-\frac{1}{2} \frac{\partial_{j} g_{00}}{g_{00}}=\Gamma_{x}{ }_{j}-\frac{1}{2} \frac{\partial_{j} g_{00}}{g_{00}}
$$

Comme $g_{00}=\xi^{2}$, nous en déduisons la formule :

$$
\Gamma_{z}{ }^{a}{ }_{j}=\stackrel{*}{\Gamma}_{i}^{i}{ }_{j}+\frac{1}{2} \frac{\partial_{j} \xi}{\xi}
$$

En portant cette valeur dans (45.2), il vient

$$
\stackrel{*}{\nabla} q^{i}+\frac{q^{i} \partial_{i} \xi}{\xi}=c \rho Z-\frac{l}{\rho} u^{i} \partial_{i} \rho .
$$

Nous utiliserons cette formule sous la forme équivalente:

$$
\stackrel{*}{\nabla}_{i}\left(\xi q^{i}\right)=\xi\left(c \rho Z-\frac{l}{\rho} u^{i} \partial_{i \rho}\right)
$$

On y voit apparaitre l'influence du champ de gravitation par la présence du facteur $\xi$, dans la conduction de la ohaleur.

De la formule de divergence (45.4) dans l'espace, il nous est possible de tirer des applications de nature globale sur des modèles d'univers.

\section{III - Applications à des modèles d'univers thermodynamiques stationnaires.}

46. Univers adiabatiques.

Un modèle d'univers est dit univers adiabatique, si le vecteur courant de chaleur ${ }^{\alpha} q^{\alpha}=-x\left(\partial \alpha \theta-Z u^{\alpha}\right)$ est partout nul. Mais le champ de température n'est pas nécessairement constant. On a alors l'équation

$$
c \rho u^{\alpha} \partial_{x} \theta-\frac{l}{\rho} u^{\alpha} \partial_{\alpha} \rho=0
$$

qui régit l'évolution thermique de la matière.

Le second principe $(\$ 24)$ donne alors :

$$
\nabla \alpha\left(\rho \sigma u^{\alpha}\right) \geq 0 \text {. }
$$


L'entropie de chaque élément de l'univers va constamment en croissant. C'est cette propriété qui doit fixer le sens des lignes de courant et des lignes de temps.

47. Uuivers adiabatique stationnaire à sections d' espace compactes orientables.

Considérons un modèle d'univers thermodynamique $U$ satisfaisant aux hypothèses suivantes :

a. - la métrique de $U$ est partout régulière, stationnaire;

b. - en dehors des domaines meublés par la matière, cette métrique satisfait aux équations du cas extérieur

$$
R_{\alpha \beta}=0 \text {. }
$$

Dans les domaines engendrés par les lignes de temps, meublés par la matière, cette métriqne satisfait anx équations du cas intérieur

$$
R_{\alpha \beta}-\frac{1}{2} R g_{\alpha \beta}=\chi T_{\alpha \beta}
$$

sans constante cosmologique.

Les deux métriques intérienre et extérieure se raccordent conformément aux conditions de SchWARZSOHILD;

c. - les sections d'espace $W_{3}$ sont des variétés compactes, orientables, orientées dans l'espace;

d. - le mouvement du milieu thermodynamique dans les domaines meubless est permanent, et adiabatique:

$$
q^{\alpha}=-x\left(\partial^{\alpha} \theta-Z u^{\alpha}\right)=0 .
$$

On notera que le théorème général sur les mouvements permanents (§ 41) s'applique à des modifications très simples près, la définition de ces mouvements restant la même.

Le vecteur courant de chaleur $q^{\alpha}$ est nul. Il n'y a pas de conduction thermique. Le tenseur d'impulsion-énérgie devient:

$$
T^{\alpha \beta}=(\rho+p) u^{\alpha} u^{\beta}-p g^{\alpha \beta} .
$$

Nous allons pouvoir démontrer le théorème suivant:

THÉoRime. - Il ne peut exister de modèle d' univers adiabatique station. naire satisfaisant aux hypothèses $a$ b $c$ c $d$.

En effet, des équations d'Einsteis, on tire :

$$
R_{0}^{0}=\chi\left(T_{0}^{0}-\frac{1}{2} T\right)=\chi\left[\rho\left(u_{0} u^{0}-\frac{1}{2}\right)+p\left(u_{0} u^{0}+\frac{1}{2}\right)\right] .
$$


Or, il vient du caractère unitaire de $u_{\lambda}$ :

$$
\begin{gathered}
u_{0} u^{0}-\frac{1}{2}=g^{00}\left(u_{0}\right)^{2}+ \\
\left.-g^{0 i} u_{0} u_{i}-\frac{1}{2}\left[g^{00} u_{10}\right)^{2}+2 g^{0 i} u_{0} u_{i}+g^{i j} u_{i} u_{j}\right] \\
=\frac{1}{2}\left[g^{00}\left(u_{0}\right)^{2}-g^{i j} u_{i} u_{j}\right] .
\end{gathered}
$$

D'après les hypothèses faites sur les sections d'espace $W_{3}, g^{i j} u_{i} u_{j}$ est définie négative. Il en résulte que la quantité $\left(u_{0} u^{\bullet}-\frac{1}{2}\right)$ est positive et il en est à fortiori de même, de la quantité $u_{0} u^{0}+\frac{1}{2}$.

$R_{0}{ }^{\circ}$ est alors strictement positif, dans les régions meublées de $W_{3}$, nul en dehors. De la relation (44.7) qui s'écrit

$$
\operatorname{div} \vec{h}=-\xi R_{0}^{\circ}
$$

on déduit par intégration sur $W_{3}$ :

$$
\iiint_{W_{3}} \xi R_{0}{ }^{\circ} d \tau=0 .
$$

Cette relation est impossible, puisque $R_{0}{ }^{0}$ est strictement positif dans certains domaines de $W_{3}$ et nul sur le complémentaire. On en déduit le théorème énoncé.

48. Comportement asymptotique euclidien.

Considérons un espace euclidien $E_{3}$ à trois dimensions, admettant une métrique définie négative $d \bar{s}^{2}$. Nous rapportons $E_{3}$ à un système de coordonnées normales $y^{i}$ pour lesquelles

$$
d \bar{s}^{*}=\delta_{i j} d y^{i} d y^{j}
$$

où $\delta_{i j}=-1$ si $i=j$ et $\delta_{i j}=0$ si $i \neq j$.

Nous dirons que l'espace-temps $V_{4}$ admet un comportement asymptotique euclidien sur $W_{3}$, lorsque pour un point $a$ de $W_{3}$ et un nombre $R$ suffisament grand:

a. - il existe un homéomorphisme $H$ de classe $C^{2}$ du domaine $d(\alpha, x)>R$ de $W_{3}$ sur un domaine de $E_{3}$ homéomorphe au complémentaire d'une boule fermée de $E_{3}$. $d(a, x)$ désigne la distance de deux points $a$ et $x$ de la variêté riemannienne $W_{3}$.

b. - si $y^{x}$ est un système de coordonnées privilegiées de $V_{4}$ défini dans le domaine de $V_{4}$ qui correspond au domaine $d(a, x)>R$ de $W_{3}$, par les $y^{4}$ et la variable $x^{0}$, nous avons dans ce domaine

$$
\left|g_{\alpha \beta}-\delta_{\alpha \beta}\right|<\frac{M}{r} \quad\left|\partial_{\gamma} g_{\alpha \beta}\right|<\frac{M}{r^{2}}
$$


où $\delta_{\alpha \beta}=0$ si $\alpha \neq \beta, \delta_{o 0}=+1, r=d(a, x), M$ est un nombre positif fixe et où les potentiels $g_{\alpha \beta}$ et leurs dérivées sont relatifs aux coordonnées privilégiées $y^{x}$.

Dans la suite, nous n'envisagerons que des éléments de la section d'espace $W_{3}$ de $\nabla_{4}$.

Soit $x_{0}$ un point de $W_{3}$ tel que $r_{0}=d(a, x)>R, y^{0}$ son image par l'homéomorphisme $H$ dans $E_{3}$ et $\rho_{0}$ la distance ordinaire de $y_{0}$ à l'origine 0 dans $E_{3}$. On démontre que:

$$
\rho_{0}<K r_{0}
$$

$K$ étant un nombre positif fixe.

Soit alors $\Sigma_{0}$ une sphère de $E_{3}$ de centre 0 et de rayon $\rho, S_{r_{0}}$ l'ensemble des points de $W_{3}$ tels que $d(a, x)=r_{0}>R$. Nous choisissons $\rho$ suffisamment grand pour que $\Sigma_{\rho}$ contienne l'image par $H$ de $S_{r_{0}}$ et désignons par $S_{\rho}$ l'image dans $W_{3}$ de la sphère $\Sigma_{\rho}$.

Considérons le compact $B_{\rho}$ de $W_{3}$ défini par:

1. - les points $x$ pour lesquels $d(a, x) \leq r_{0}$.

2. - les points $x$ pour lesquels $d(a, x) \geq r_{0}$, dont l'image dans $E_{3}$ est intérieure à la sphère $\Sigma_{\rho}$ ou sur cette sphère.

Le bord de $B_{\rho}$ est $S_{\rho}$ orientée vers l'extérieur.

$\vec{p}$ désigne le vecteur introduit au $\S 44$, de composantes

$$
p_{i}=\frac{\xi^{3}}{2} \varphi_{k} H_{i}{ }^{k}
$$

On se propose d'étudier le comportement de son flux à travers $S_{\rho}$ quand $\rho \rightarrow \infty$.

Pour cela évaluons le module $p d u$ vecteur $\vec{p}$. Dans un repère orthornomé de $W_{3}$ :

$$
p_{i}=-\frac{\xi^{3}}{2} \sum_{k} \varphi_{\underline{k}} H_{\underline{i} \underline{k}} .
$$

D'après l'inégalité de ScHwarTz:

$$
\left(p_{i}\right)^{2} \leq\left(\frac{\xi^{3}}{2}\right)^{2} \sum_{k}\left(\varphi_{k}\right)^{2} \sum_{j}\left(H_{i}\right)^{2}
$$

on en déduit:

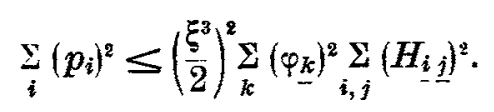

En introduisant les modules $\varphi$ et $H$ des tenseurs $\varphi_{k}$ et $H_{i j}$, on a :

$$
p \leq \frac{1}{\sqrt{2}} \xi^{3} \varphi H .
$$


Le flux de $\vec{p}$ à travers $S_{\rho}$ peut alors être majoré par:

$$
\left|\operatorname{flux}_{S_{\rho}}^{*} \vec{p}\right| \leq \iint_{S_{\rho}} p d \stackrel{*}{*} \leq \frac{1}{V \overline{2}} \iint_{S_{\rho}} \xi^{3} \varphi H d \sigma
$$

où $d_{\sigma o ́}^{*}$ est l'élément d'aire de $S_{\rho}$ dans la métrique $d^{*} s^{2}$.

Sous nos hypothèses, on a sur $S$ :

$$
\xi^{3} \varphi H<\frac{C_{4}}{r^{3}}
$$

où $r=d(a, x)$ et $C_{1}$ est un nombre fixe. Il en résulte d'après (48.3) qu'il existe un nombre fixe $C_{2}$ tel que

$$
\xi^{g} \varphi H<\frac{C_{2}}{\rho^{3}} .
$$

D'autre part

$$
\iint_{S_{\rho}} d \sigma=\iiint_{S_{\rho}}\left[1+0\left(\frac{1}{r}\right)\right] d \vec{\sigma}=C_{3} \iint_{\Sigma_{\rho}} d \bar{\sigma}
$$

où $d \bar{\sigma}$ désigne l'aire euclidienne et $C_{3}$ est un nombre fixe. Il vient ainsi

$$
\left|\underset{S_{f}}{\operatorname{flux}} \vec{p}\right|<\frac{1}{\sqrt{2}} \frac{C_{2}}{\rho^{3}} C_{3} 4 \pi \rho^{2}<\frac{C}{\rho}
$$

$C$ étant un nombre fixe.

On en déduit la formule

$$
\lim _{\rho \rightarrow \infty} \operatorname{flux}_{S_{\rho}}^{*} \vec{p}=0
$$

49. Univers thermodynamique stationnaire à comportement asymptotique euclidien.

Considérons un modèle d'univers thermodynamique stationnaire $U$ satisfaisant aux hypothèses suivantes:

a. - la métrique de $U$ est partout régulière, stationnaire. L'espacetemps associé admet une section d'espace $W_{3}$ complète et à comportement asymptotique euclidien dans le domaine à l'infini de $W_{3}$.

$b$. - les distributions énergétiques sont à distance finie et occupent dans $W_{3}$ des domaines $D_{4}$. La température $\theta$ tend uniformément vers une constante dans le domaine à l'infini de $W_{3}$.

c. - le milieu matériel remplissant les domaines $D_{4}$ est supposé être un fluide parfait thermodynamique, obéissant au équations sans constante cosmologique

$$
S_{\alpha \beta} \equiv R_{\alpha \beta}-\frac{1}{2} R g_{\alpha \beta}=\chi T_{\alpha \beta}
$$

ec animé d' un mouvement permanent. 
Un tel modèle d'univers thermodynamique stationnaire sera dit plus simplement à comportement asymptotique euclidien.

Nous ferons l'hypothèse

$$
Z \equiv u^{\alpha} \partial_{\alpha} \theta=0 \quad u^{\alpha} \partial_{\alpha} \rho=0
$$

e'est-à-dire que la température et la densité restent constantes le long des lignes de courant, $x$ est supposé constant. L'équation de conduction thermique se réduit alors à

$$
\operatorname{div}(\xi) \overrightarrow{\operatorname{rad}} \theta)=0 .
$$

Nous pouvons l'écrire sous la forme:

$$
\begin{aligned}
& L(\theta) \equiv g^{i j} \partial_{i j} \theta+h^{k} \partial_{k} \theta=0 \\
& h^{k}=-g^{i j \Gamma_{i}^{*}{ }_{j}+g^{i k} \partial_{i} \xi}
\end{aligned}
$$

où par hypothèse $\theta$ est une fonction de classe $C^{2}$, los $g_{\alpha \beta}, \xi$ sont de classe $C^{1}$. $h^{k}$ est donc continue.

$\theta$ tend uniformément vers une constante $K$ dans le domaine à l'infini de $W_{3}$. Cela signifie que, $a$ désignant un point déterminé, à tout $\varepsilon$ on peut associer un nombre $R$ ne dépendant que de $\varepsilon$, tel que

$$
d(a, x) \geq R \text { entraîne }|\theta(x)-K|<\varepsilon .
$$

Alors, ou bien $\theta(x)=K$ sur $W_{3} ;$ ou bien $\theta(x)$ prend sur $W_{3}$ des valeurs différentes de $K$. Supposons par exemple qu'il existe un point $x_{0}$ tel que:

$$
\theta\left(x_{0}\right)>K
$$

(on traiterait de même l'hypothèse contraire en raisonnant sur $-\theta$ ). On a :

$$
\theta(x)<K+\varepsilon<\theta\left(x_{0}\right) \text {. }
$$

Considérons le domaine $C_{R}$ defini par $d(a, x) \leq R$. Il contient $x_{0}$ à son intréieur. Le maximum de $\theta(x)$ sur $C_{R}$ est done atteint en un point intérieur $b$ et l'on a:

$$
\begin{array}{llc}
\text { pour } & d(a, x) \leq R & \theta(x) \leq \theta(b) \\
\text { pour } & d(a, x) \geq R & \theta(x)<\theta\left(x_{0}\right) \leq \theta(b)
\end{array}
$$

$\theta(x)$ atteint done son maximum au point $b$.

Revenons maintenant à (49.3). La forme quadratique des coefficients $g^{i j}$ est définie négative, $\theta$ est de classe $C^{2}$ et $h^{k}$ continue. Nous pouvons appliquer le théorème de HoPF $\left(^{6}\right)$ et en déduire que $\theta$ est une constante sur $W_{3}$.

Nous énonçons dono ce premier résultat.

THÉORÈmE. - Dans tout modèle d'univers thermodynamique stationnaire à comportement asymptotique euclidien, si la température $\theta$ et la densité $\rho$ restent constantes le long des lignes de courant, le champ de température $\theta$ est constant.

(6) E. Hopf, ‘ Preuss. Akad. Wiss », 19, 147.152, (1927). 
Pham MaU Quan: Sur une théorie relativiste des fluides, etc.

203

En particulier si les lignes de courant coüncident avec les lignes de temps, on a aussi $u^{\alpha} \partial_{x} \theta=u^{\alpha} \partial_{x} \rho=0$ et le champ de température est constant.

Oonsidérons le compact $B_{p}$ tel qu'il a été défini dans le paragraphe précédent. Appliquons à $B_{\rho}$ la formule (40.10). Pour $\rho$ suffisamment grand, il vient

$$
\iiint_{B_{\rho}} \xi \varphi_{i} R_{0}{ }^{i} d \xi=\iiint_{B_{\rho}} \frac{\xi^{3}}{2} H^{2} d \tau-\underset{S_{\rho}}{*} \underset{\mathbf{p}}{*} \vec{p}
$$

$R_{0}{ }^{i}$ étant nul en dehors des domaines $D_{u}$, nous avons la relation

$$
\underset{v}{\Sigma} \iiint_{D_{w}} \xi \varphi_{i} R_{0}{ }^{i} d \tau=\iiint_{B_{\rho}}^{\xi^{3}} \frac{\xi^{2}}{2} H^{2} d \tau-\underset{S_{\rho}}{*} \underset{\mathbf{n} x}{*} \vec{p}
$$

Lorsque $\rho \rightarrow \infty$, le flux de $\vec{p}$ tend vers 0 d'après la formule (48.4). $L$ 'intégrale du second membre est convergente. On a :

$$
\iiint_{W_{3}}^{\xi^{3}} \frac{{ }^{3}}{2} H^{2} d \tau=\underset{u}{\Sigma} \iiint_{D_{u}} \xi \varphi_{i} R_{0}^{i} d \tau
$$

Des équations d' Einsters, on tire

$$
R_{0}^{i}=S_{0}^{i}=\chi\left[(\rho+p) u_{0}^{i} u^{i}-p g_{0}^{i}-\left(u_{0} q^{i}+u^{i} q_{0}\right)\right] .
$$

Si les lignes de courant coïncident avec les lignes de temps, dans notre système de coordonnées adaptées, $u^{i}=0$ et d'après le théorème précédent $\theta=$ const, $q^{i}=0$. 11 en résulte que $R_{0}{ }^{i}$ est nul et la relation (49.5) se réduit à

$$
\iiint_{W_{3}} \frac{\xi^{3}}{2} H^{2} d \tau=0 .
$$

L'élément d'intégration est positif ou nul. Il est nécessaire que $H^{2} \equiv 0$ sur $W_{3}$. L'espace-temps est tel que les lignes de temps sont orthogonales a une famille, de sections d'espace locales. Un tel modèle d'univers est un espace-temps statique au sens de LEVI-CIVITA.

Nous avons établi le théorème suivant qui constitue une extension d'un théorème global connu [18].

ThÉonìime. - Tout modèle d'univers thermodynamique stationnaire à comportement asymptotique euclidien, pour lequel les lignes de courant de la matière (supposée schématisée sous forme de fluide parfait) coïncident avec les lignes de temps, est partout statique au sens de Levi-Civita. Le champ de température $y$ est constant. 


\section{BIBLIOGRAPHIE}

[1] E. Gartan, Legons sur la geométrie des espaces de Riemann, Gauthier-Villars, 1946.

[2] E. CARtAN, Sur les équations de la gravitation $d^{\prime}$ Einstein, "Journ. Math. *, t. 1, 1922, p. 141.203.

[3] J. Chazy. La théorie de la relativité et la mécanique céleste, Gauthier-Villars, 1930.

[4] G. Darmors, Les équations de la gravitation einsteinienne, "Mémorial des Sc. Math. ", fasc. XXV, 1927.

[5] P. Duhem, Hydrodynamique. Elasticité. Acoustique, Cours de Physique mathematique et de eristallographie à la Faculté des Soiences de Lille, Herrmann, 1891.

[6] D. van Dantzig, On relativistic thermodynamics, a Proc. Kond. Ned. Akad. v. Wetensch. * Amsterdam 42, 601.607, 1939.

[7] D. van Dantzig, On the thermohydrodynamics of perfectly perfect fluid, Ib., 43, 1940.

[8] C. ECKART, The thermodynamics of irreversible process. III, Relativistic theory of the simple fluid, " Phys. Rev. *, 58, 1940.

[9] Einsten, Sitzungsb, Berlin, 1918, p. 155-167. Quatre conférences sur la théorie de la relativité faites a l'université de Princeton (trad. M. Solovine, Gauthier-Villar's, 1925).

[10] Ch. FaBry, Eléments de thermodynamique, A. Colin, 1950.

[11] Mme Y. Foures-Bruhat, Théorème d'existence pour certains systèmes d'èquations aux dérivées partielles non linéaires, Acta Mathematica ", 88, p. 141.225, 1952.

[12] Fourigr, Theorie analytique de la chaleur in Oenvres de Fourier, Gauthier-Villars, 1888.

[13] H. Hugoniot, Mémoire sur la propagation au mouvement dans les corps et spécialement dans les gaz parfaits, * J. de l'École Polytechnique s, 57è cah. 1877, 58ð cah. 1889.

[14] A. Lichnerowroz, Problemes globaux en mécanique relativiste "Act. Sc. Ind. 833 ", Herimann, Paris, 1939.

[15] A. Lichnerowraz, Sur l'invariant intégral de l'hydrodynamique relativiste, *Ann. Ecole normale superienre ", fasc. IV, 1941.

[16] A. Lichnerowroz, Algèbre linéaire, Masson, 1947.

[17] A. Lichnerowicz, Elements de calcul tensoriel, A. Colin, 1950.

[18] A. Lichnerowiaz, Etude mathématique des théories relativistes de la gravitation et de l'électromagnétisme (cours professé au Collège de France) (sous presse). I. - Relativité générale classique (1952-53). II. - Théories unitaires (1953-54).

[19] Pham Mau Quan, Notes aux C. R. Acad. des Sc., t. 286, p. 2299-2301 (1953), t. 237, p. 22.24 (1953), t. 238, p. $324-325$ (1954).

[20] R. Tolman, Relátivity, Thermodynamics and Cosmology, Oxford, 1950.

[21] A. H. TAUB, Relativistic Rankine Hugoniot equations, "Physical Rov. „, 74, 1948, p. 328. 